\title{
Adaptive Random Linear Network Coding with Controlled Forwarding for Wireless Broadcast
}

\author{
by \\ Kashif Mahmood \\ A thesis submitted to The Faculty of Graduate Studies and Research \\ in partial fulfilment of the degree requirements of \\ Master of Applied Science in Electrical and Computer Engineering
}

Ottawa-Carleton Institute for Electrical \& Computer Engineering

Department of Systems and Computer Engineering

\author{
Carleton University \\ Ottawa, Ontario, Canada \\ December 2010
}

(C) Copyright 2010, Kashif Mahmood 
The undersigned recommend to

the Faculty of Graduate Studies and Research acceptance of the thesis

\title{
Adaptive Random Linear Network Coding with Controlled Forwarding for Wireless Broadcast
}

\author{
Submitted by Kashif Mahmood \\ in partial fulfilment of the requirements for the degree of \\ Master of Applied Science in Electrical and Computer Engineering
}

(Dr. Thomas Kunz), Thesis Co-Supervisor

(Dr. Ashraf Matrawy), Thesis Co-Supervisor

(Dr. Howard Schwartz), Chair, Department of Systems and Computer Engineering

Carleton University

2010 


\begin{abstract}
Multicasting and broadcasting are important communication techniques in wireless adhoc networks. Recently, Network Coding (NC), which has emerged as a promising technique for various applications, has been applied to multicast and broadcast in wireless adhoc networks. It is however observed that the performance using NC is strongly dependent upon the topology, node density and the kind of coding algorithm. The algorithms that are proposed are mostly dealing with single source multicasting or broadcasting.

In this thesis I propose an adaptive multi-source broadcasting protocol using Random Linear Network Coding (RLNC). The key features of this protocol include its multi-source operation, cross-session generations, controlling the number of retransmissions effectively based on neighbourhood information and earlier decoding. Our simulations with and without cross-session generations show that cross-session generations result in improved Packet Delivery Ratio as well as lower latency. We also investigate its adaptive performance compared to packet forwarding schemes, including a simple flooding protocol, a probabilistic flooding protocol, BCAST and Simplified Multicast Forwarding. We observe the steady performance of our protocol under different node densities and rates.
\end{abstract}


Dedicated to my parents, my wife and my wonderful son 


\section{Acknowledgements}

I would like to express my sincere gratitude to my advisors, Dr. Thomas Kunz and Dr. Ashraf Matrawy for their extraordinary support and guidance throughout my graduate studies at Carleton University. I must unequivocally acknowledge that Dr. Thomas was a real source of motivation for me and there was not a single moment when I found myself unguided. He was always available with his valuable suggestions and guidance throughout my thesis. Dr. Ashraf was also always motivating and supported me on quite a few occasions where I was in a fix.

I also would like to thank my friends and colleagues who were always there to extend support and motivation. I would like to thank the SCE department and overall the Carleton University for all their support. This work was partially funded by Communication Research Center (CRC), Canada and I would like to thank CRC for their support. I also would like thank the Graduate Chair for all his moral and financial support.

Not to mention, I would like to especially thank my parents whose prayers are always there for me and it's due to their prayers and efforts which have led me to success in life. I would like to thank my wife and son, who were always there to support me in all circumstances and situations. As a student with minimal resources and no time for family, she was always there as a support. Finally, I would like to thank my sister and brother-in-law who always motivated me and guided me on all occasions during the research work. 


\section{Table of Contents}

Chapter 1 Introduction ......................................................................................1

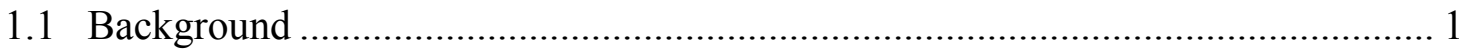

1.2 Emergence of Network Coding for Wireless Networks......................................... 1

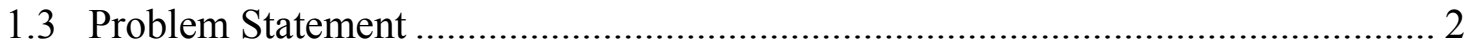

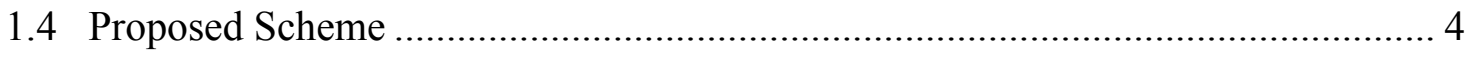

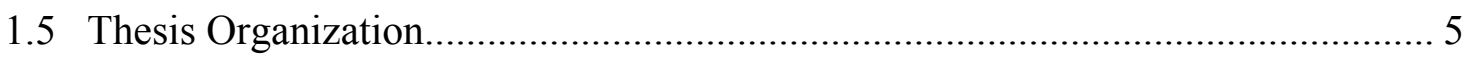

Chapter 2 Network Coding....................................................................6

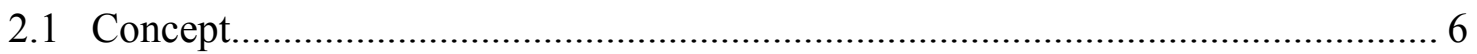

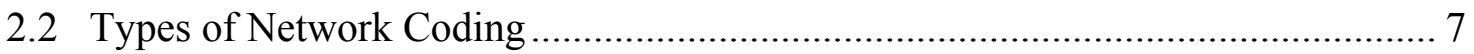

2.2.1 XOR-based NC .................................................................................... 7

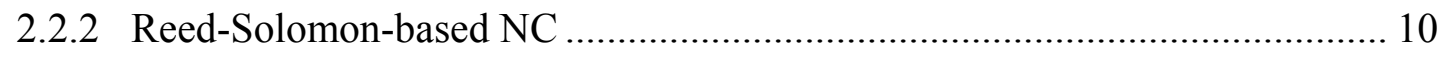

2.2.3 Random Linear Network Coding …………............................................ 12

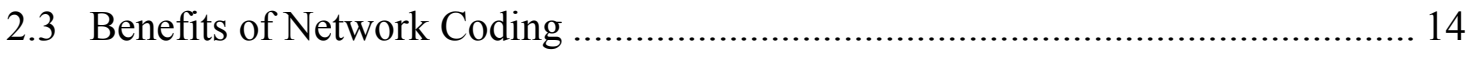

Chapter 3 Related Work ...........................................................................17

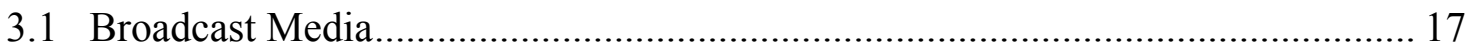

3.2 Efficient Packet-Forwarding-Based Wireless Broadcast ...................................... 18

3.3 Related Work on RLNC for Wireless Networks.................................................. 20

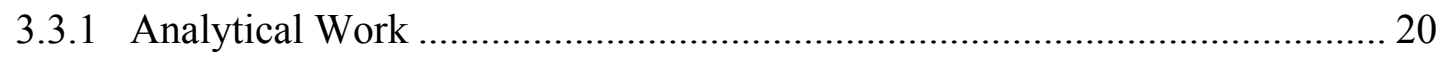




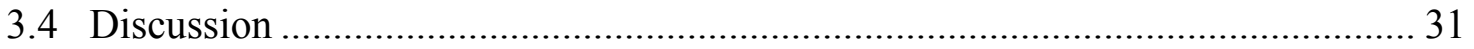

\section{Chapter 4 Proposed Model ......................................................................34}

4.1 Adaptive Random Linear Network Coding with Controlled Forwarding

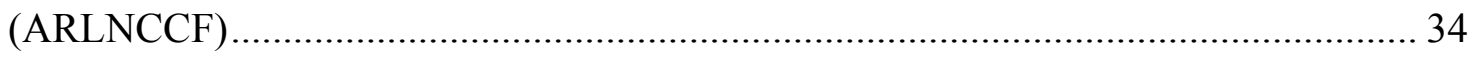

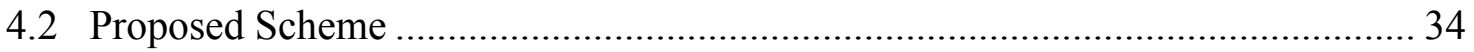

4.2.1 Hello Control Messages and Number of Retransmissions ........................... 36

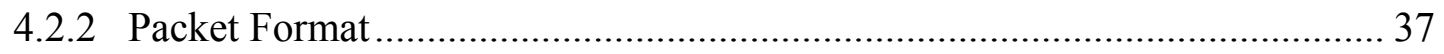

4.2.3 Generation Size.................................................................................. 39

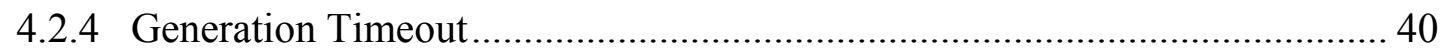

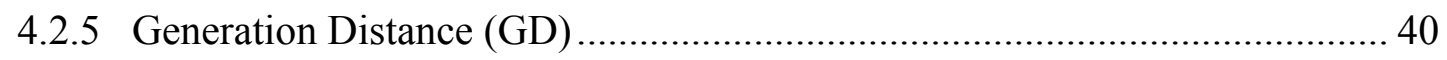

4.2.6 Partial and Full Decoding ................................................................. 41

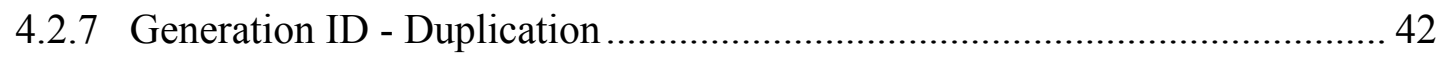

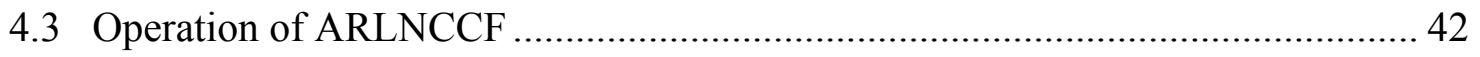

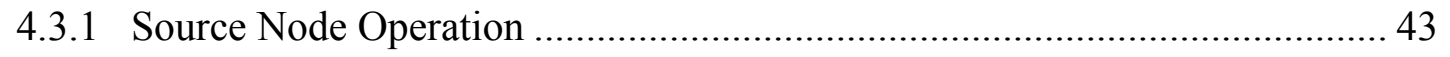

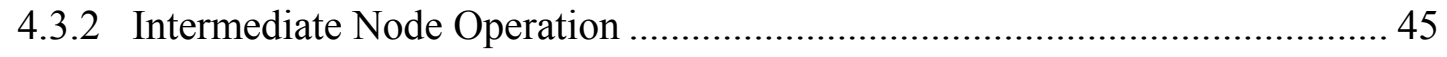

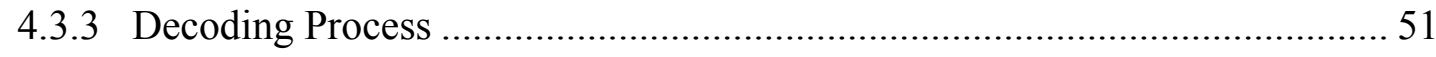

Chapter 5 Simulation Setup...............................................................55

5.1 Simulation Tool \& Parameters ...................................................................... 55

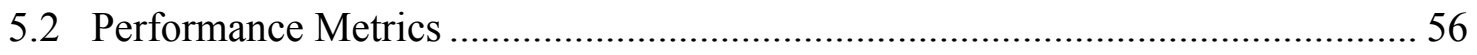


5.3 Algorithms for Comparison ...................................................................... 58

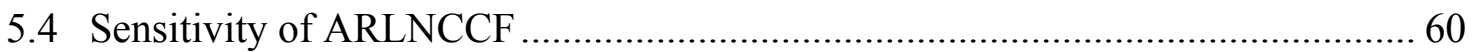

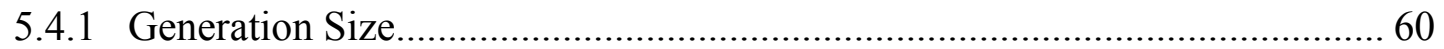

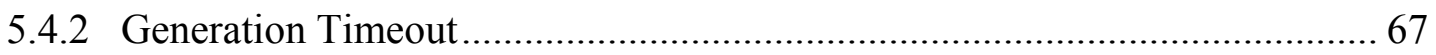

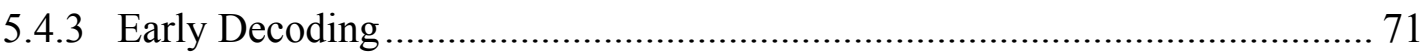

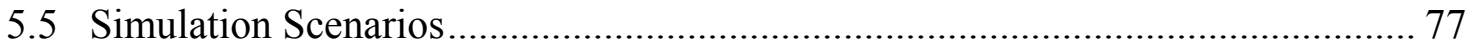

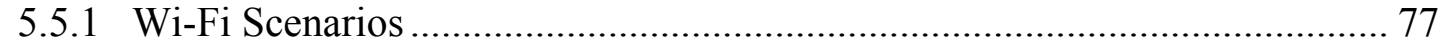

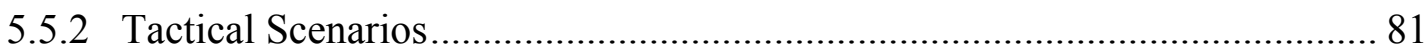

Chapter 6 Adaptive Performance ...............................................................84

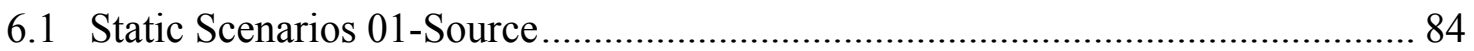

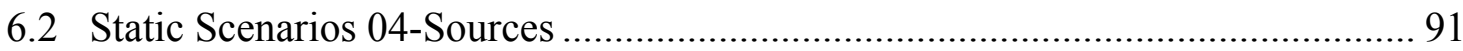

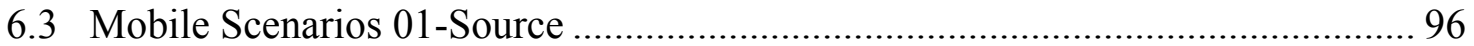

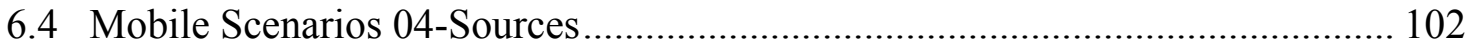

6.5 Tactical Scenarios 01-Source .................................................................. 106

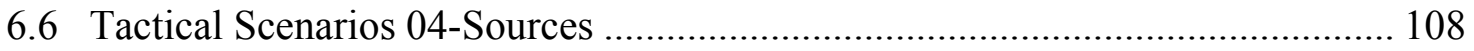

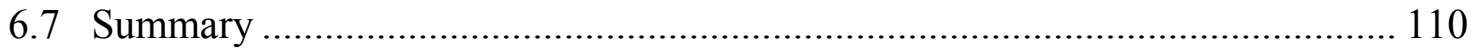

\section{Chapter 7 Cross-Session Performance ........................................................114}

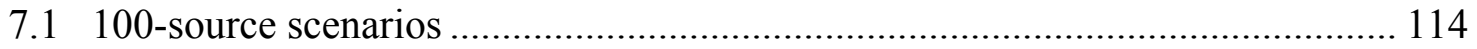

7.2 4-Source Scenarios .............................................................................. 116

Chapter 8 Conclusions and Future Work ...........................................121 


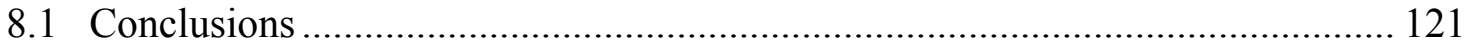

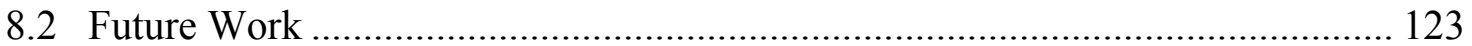

Appendix A................................................................................................132 


\section{List of Figures}

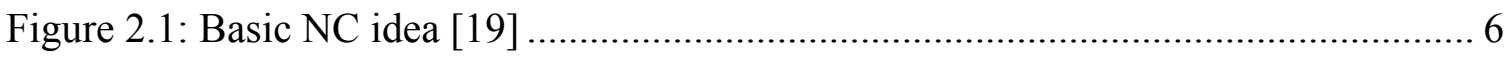

Figure 2.2: Butterfly Network without NC ............................................................... 7

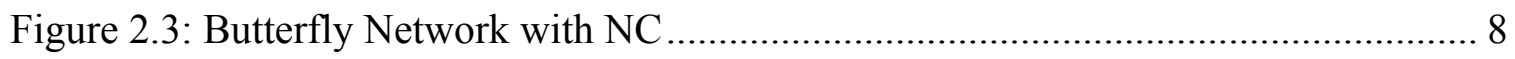

Figure 2.4: Data Forwarding without and with COPE [20].......................................... 9

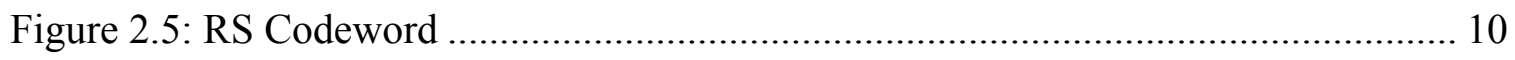

Figure 2.6: RLNC Process …………………………....................................... 12

Figure 3.1: Dominating Set (DS) \& Connected Dominating Set (CDS) .......................... 19

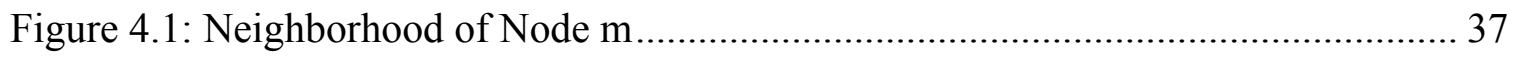

Figure 4.2: Packet Format for ARLNCCF..................................................................... 38

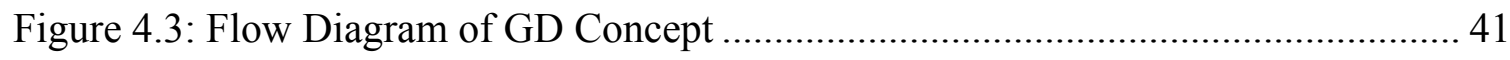

Figure 4.4: Flow Diagram - Encoding Process ........................................................... 43

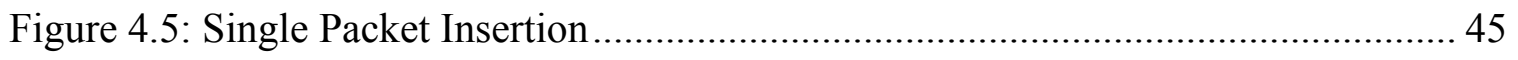

Figure 4.6: Flow Diagram - Intermediate Node Operation.............................................. 46

Figure 4.7: Generation with Symbol Location and Respective Coding Coefficients....... 48

Figure 4.8: Received Packet with Symbol Location and Respective Coefficients........... 49

Figure 4.9: Generation 73098 after Conflict Resolution .............................................. 49

Figure 4.10: Generation 73098 with Symbol Location and Respective Symbols ............ 50

Figure 4.11: Received Packet for Generation 73098 ..................................................... 51

Figure 4.12: Generation 73098 after Conflict Resolution ............................................... 51

Figure 4.13: Flow Diagram - Decoding Process ......................................................... 52

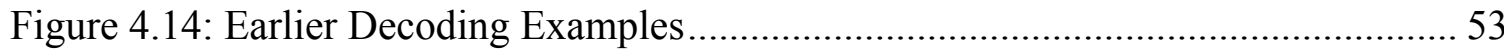

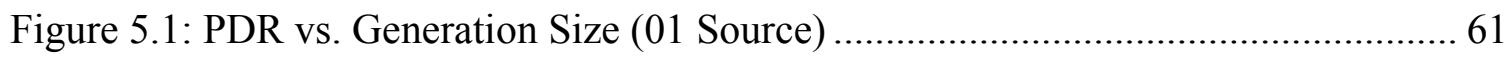




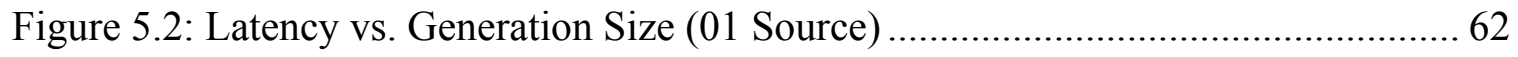

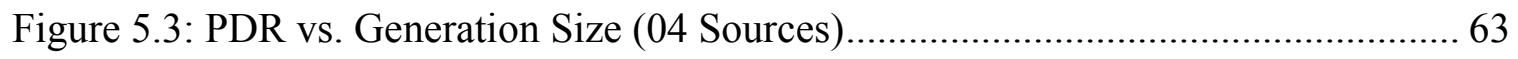

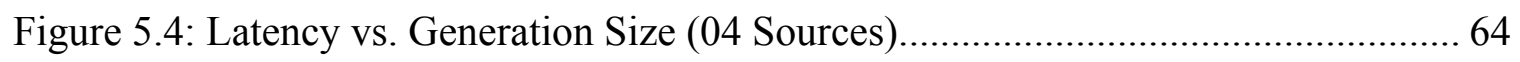

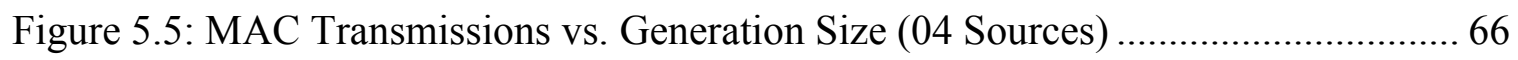

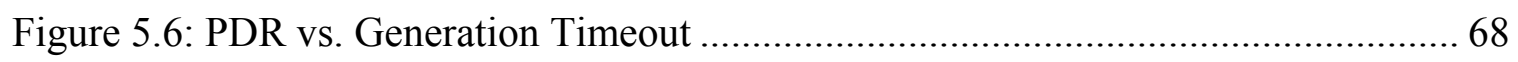

Figure 5.7: Latency vs. Generation Timeout ......................................................... 70

Figure 5.8: MAC Transmissions vs. Generation Timeout ....................................... 70

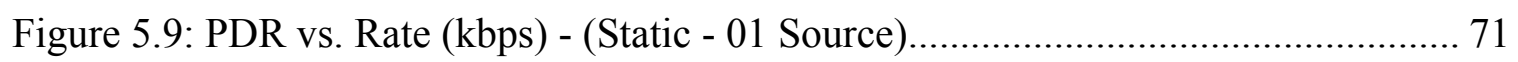

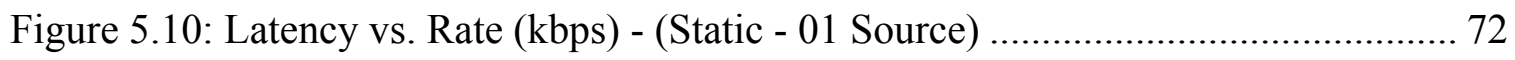

Figure 5.11: PDR vs. Nodes (Static - 01 Source) …............................................... 73

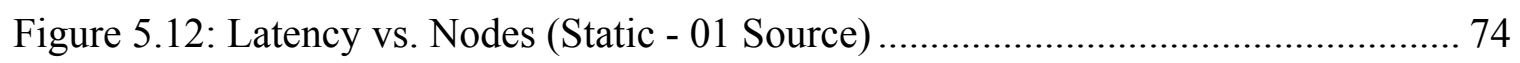

Figure 5.13: PDR vs. Rate (kbps) - (Static - 04 Source) ............................................ 75

Figure 5.14: Latency vs. Rate (kbps) - (Static - 04 Source) ......................................... 75

Figure 5.15: PDR vs. Nodes (Static - 04 Sources) …........................................... 76

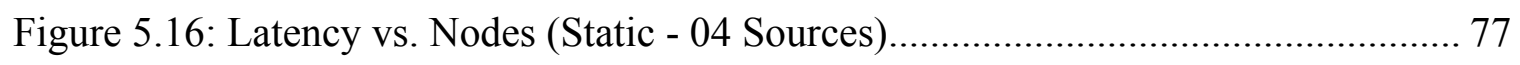

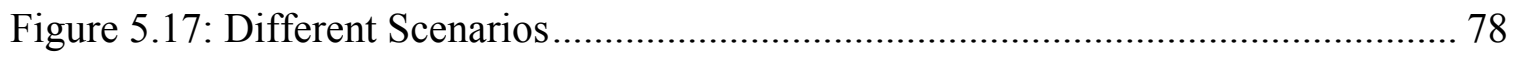

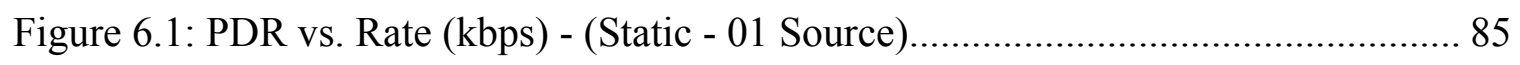

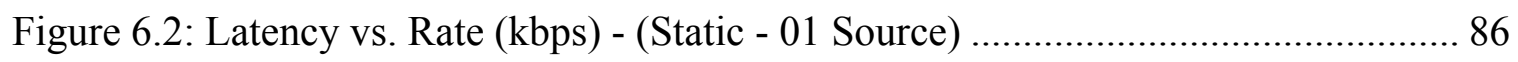

Figure 6.3: MAC Transmissions vs. Rate (kbps) - (Static - 01 Source) ........................... 86

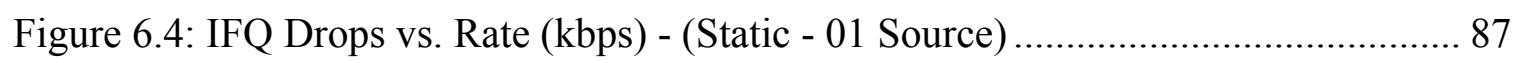

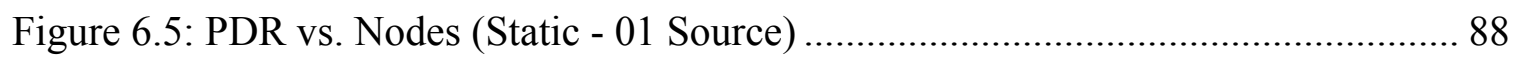

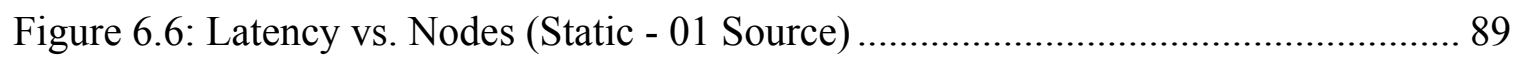

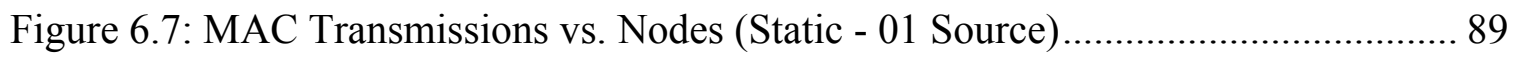


Figure 6.8: IFQ Drops vs. Nodes (Static - 01 Source) ............................................... 90

Figure 6.9: PDR vs. Rate (kbps) - (Static - 04 Sources) ........................................... 91

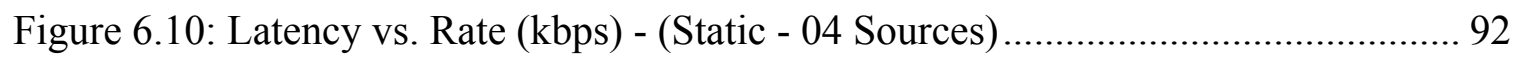

Figure 6.11: MAC Transmissions vs. Rate (kbps) - (Static - 04 Sources)....................... 92

Figure 6.12: IFQ Drops vs. Rate (kbps) - (Static - 04 Sources) ................................... 93

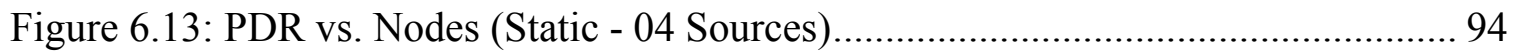

Figure 6.14: Latency vs. Nodes (Static - 04 Sources) ............................................. 95

Figure 6.15: MAC Transmissions vs. Nodes (Static - 04 Sources) ............................... 95

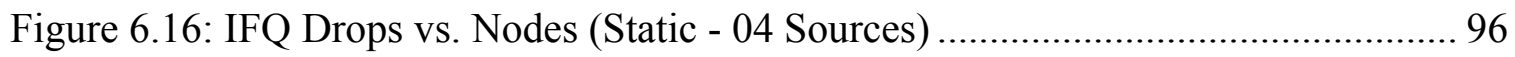

Figure 6.17: PDR vs. Rate (kbps) - (Mobility - 01 Source) .......................................... 97

Figure 6.18: Latency vs. Rate (kbps) - (Mobility - 01 Source) ....................................... 97

Figure 6.19: MAC Transmissions vs. Rate (kbps) - (Mobility - 01 Source) .................... 98

Figure 6.20: IFQ Drops vs. Rate (kbps) - (Mobility - 01 Source) ................................ 98

Figure 6.21: PDR vs. Nodes - (Mobility - 01 Source) …........................................ 100

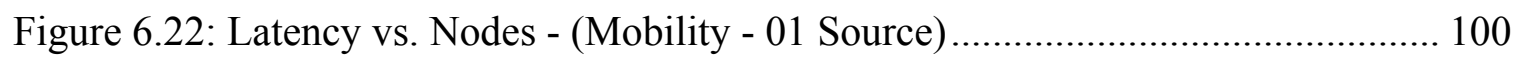

Figure 6.23: MAC Transmissions vs. Nodes - (Mobility - 01 Source).......................... 101

Figure 6.24: IFQ Drops vs. Nodes - (Mobility - 01 Source) ....................................... 101

Figure 6.25: PDR vs. Rate (kbps) - (Mobility - 04 Sources) ....................................... 102

Figure 6.26: Latency vs. Rate (kbps) - (Mobility - 04 Sources) ................................. 103

Figure 6.27: MAC Transmissions vs. Rate (kbps) - (Mobility - 04 Sources)................. 103

Figure 6.28: IFQ Drops vs. Rate (kbps) - (Mobility - 04 Sources)............................... 104

Figure 6.29: PDR vs. Nodes - (Mobility - 04 Sources) …........................................ 104

Figure 6.30: Latency vs. Nodes - (Mobility - 04 Sources) …................................... 105 
Figure 6.31: MAC Transmissions vs. Nodes - (Mobility - 04 Sources) ......................... 105

Figure 6.32: IFQ Drops vs. Nodes - (Mobility - 04 Sources) ..................................... 106

Figure 6.33: PDR vs. Rate (kbps) - (Tactical - 01 Source) ......................................... 107

Figure 6.34: Latency vs. Rate (kbps) - (Tactical - 01 Source) .................................... 107

Figure 6.35: MAC Transmissions vs. Rate (kbps) - (Tactical - 01 Source) ................... 108

Figure 6.36: PDR vs. Rate (kbps) - (Tactical - 04 Sources) ....................................... 109

Figure 6.37: Latency vs. Rate (kbps) - (Tactical - 04 Sources) .................................. 109

Figure 6.38: MAC Transmissions vs. Rate (kbps) - (Tactical - 04 Sources) .................. 110

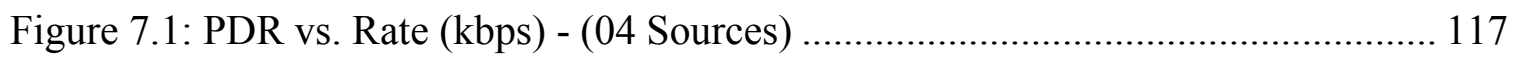

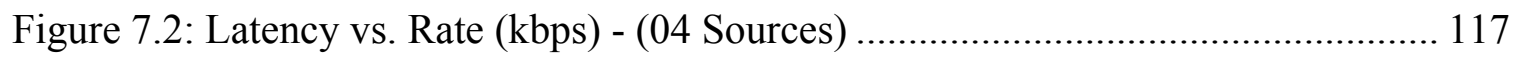

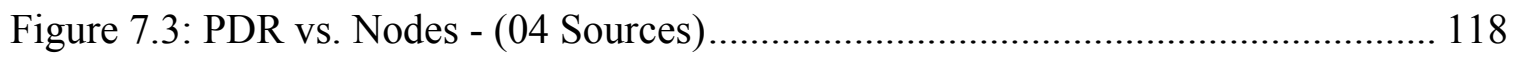

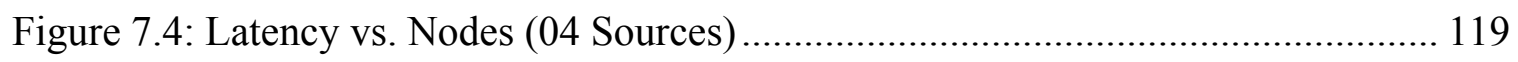

Figure A.1: Encoding Process............................................................................... 132 


\section{List of Tables}

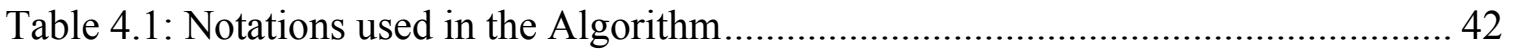

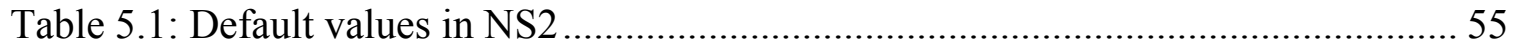

Table 5.2: Optimal P values used for Probabilistic Flooding ............................................ 59

Table 5.3: Common Parameters................................................................................. 79

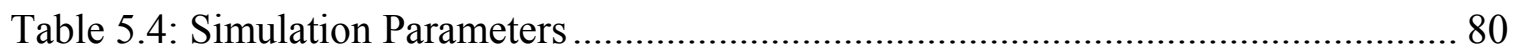

Table 5.5: Simulation Parameters .............................................................................. 81

Table 6.1: Comparing SMF and ARLNCCF for Wi-Fi and Tactical Scenarios............. 111

Table 7.1: PDR for 1 Packet and 4 Packets per Source ………………….................... 115

Table 7.2: Latency for 1 Packet and 4 Packets per Source............................................. 116

Table 7.3: Confidence Interval-PDR (04 Sources - 50 Nodes) ……………………...... 118

Table 7.4: Confidence Interval-Latency (04 Sources - 50 Nodes) ................................. 119

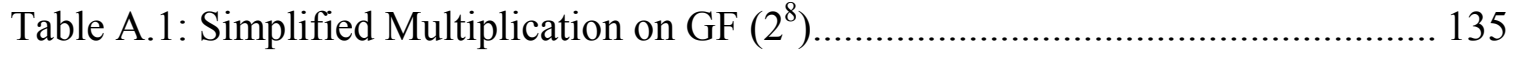




\section{List of Acronyms}

AES: Advanced Encryption Standard

ARLNCCF: Adaptive Random Linear Network Coding with Controlled Forwarding

ARQ: Automatic Repeat reQuest

ARQ-E: Enhanced ARQ

ARQ-SPR: Single Path Routing ARQ

BCAST: No Acronym

CBR: Constant Bit Rate

CDS: Connected Dominating Set

DS: Dominating Set

DTN: Delay Tolerant Networks

FEC: Forward Error Correction

GD: Generation Distance

GF: Galois Field

MANETs: Mobile Adhoc NETworks

$\mathrm{MC}^{2}$ : Multipath Code Casting

MPR: Multi Point Relay

NC: Network Coding

OLSR: Optimized Link State Routing protocol

OMNC: Optimized Multipath Network Coding

PDR: Packet Delivery Ratio

RLNC: Random Linear Network Coding

RS: Reed-Solomon 
SMF: Simplified Multicast Forwarding

S-MPR: Source based Multi Point Replay

TBRPF: Topology dissemination Based on Reverse-Path Forwarding 


\section{Chapter 1}

\section{Introduction}

\subsection{Background}

In many wireless applications, there is a requirement to flood information to all the nodes in the network with one-to-many or many-to-many communication patterns to disseminate control messages and other important information like emergency messages in battlefield operation and disaster relief operations, etc. Simple flooding, in which each node in the network rebroadcasts the packet it receives, requires no overhead but consumes lots of channel bandwidth as many duplicate packets are received by the nodes. The result, called broadcast storm [1], causes significant packet loss and network congestion.

In order to deal with the broadcast storm problem, more efficient ways have been proposed for broadcasting in multi-hop wireless networks by reducing the number of redundant retransmissions. Various techniques have been applied to reduce the number of retransmissions (i.e. number of nodes forwarding the broadcast packets) in wireless networks while attempting to ensure that a broadcast packet is delivered to each node in the network. The detailed explanation of these techniques is provided in Chapter 3.

\subsection{Emergence of Network Coding for Wireless Networks}

Wireless networks, which are basically broadcast in nature, have some potential challenges compared to wired networks. The challenges include low throughput, limited 
bandwidth, dead spots, poor performance under mobility, energy-constrained operation, unreliability, susceptible to environmental factors such as fading and interference, and security threats. However, the inherent characteristics of wireless media like its broadcast nature, the diversity of information and data redundancy can help in designing new ways of wireless communication [2]. One emerging area, originally designed for wired networks, is Network Coding (NC) [3] that works very well in wireless broadcast environment by exploiting these characteristics. With network coding, the sending nodes or the intermediate nodes not only act as relay but they additionally combine (encode) a number of packets they have received into one or several outgoing packets, thus improving the throughput of the network. Various analytical models and simulations have shown that network coding can improve the efficiency, throughput, complexity, robustness and security of the network [4][5][6].

Various NC based techniques have been proposed and applied to applications like multicasting and broadcasting in wireless networks, peer to peer file distribution [7], security and robustness to attacks [8], video surveillance [9], as an alternative to Automatic Repeat reQuest (ARQ) [10], large scale content distribution [11], on chip communication [12] and distributed storage [13]. A more detailed explanation of these techniques is given in Chapter 2.

\subsection{Problem Statement}

In simple packet forwarding, if the packet is lost, there is no way to recover the lost packet unless the source sends that packet again or same packet is overheard from another neighbour (opportunistic listening). Using network coding, nodes are allowed to 
process (encode / re-encode) the received incoming packets instead of simply forwarding or repeating them. Thus, the packets which are independently generated by the source nodes are not required to be processed separately by intermediate nodes. These packets can be combined into one or several outgoing packets. If the encoded packet is lost, there is still a chance that if the required number of encoded packets can be collected by a node from any neighbour or group of neighbours, the original packets can be recovered without any retransmission from the source node. Due to this appealing property, network coding is able to offer benefits in various aspects of communication networks. The benefits of using NC are discussed in detail in Chapter 2.

Consideration of the benefits of NC motivated us to explore its potential for wireless broadcasting. Although there are many proposed schemes dealing with single source wireless broadcast using $\mathrm{NC}$, only few works are related to multi-source broadcast. Similarly, the protocol performance depends on how it adapts to the different data rates and node densities. Many existing protocols have parameters that can be set to appropriate values like probability of retransmission or forwarding factor [14][15] to adapt themselves to the above situations. We would like a solution where the protocol is able to adapt itself automatically, thus, making it more suitable for wireless adhoc networks.

In this thesis, we have developed a NC-based broadcast protocol that works well for both single-source and multi-source environment. We have explored the potential benefit of allowing packets originating from different sources to be combined/coded together, as opposed to the already proposed algorithms that limit this to packets originating from the same source only (detail is provided in later chapters). We have 
shown through simulations that our protocol is able to adapt well to different nodes densities and data rates and show steady performance in terms of Packet Delivery Ratio (PDR) and end-to-end packet delay.

\subsection{Proposed Scheme}

Our main focus is the application of $\mathrm{NC}$ in the area of broadcasting in wireless adhoc networks. Various analytical models have been proposed and their performance is evaluated [16][17]. Our proposed scheme uses Random Linear Network Coding (RLNC) for wireless broadcast. We call it Adaptive Random Linear Network Coding with Controlled Forwarding (ARLNCCF). Very little work addresses the issue of multi-source RLNC-based broadcast [16]. The key elements of our protocol are as following.

1. The algorithm is specifically designed to work in multi-source broadcast environments. The scheme works well for both single-source and multi-source environments by allowing to code/combine packets originating from different sources. This improves the Packet Delivery Ratio and reduces the latency.

2. Using neighbour knowledge and the generation size, our scheme can effectively calculate the number of rebroadcasts that are sufficient for all the nodes to decode the coded packets. Hence, it is adaptive to varying nodes densities, making it more suitable for adhoc networks in which there is no control over the number and density of nodes in the network.

3. The Generation Distance (GD) concept is introduced to check the size of generation to grow uncontrolled in a multi-source environment. 
4. An early decoding concept, which is also considered in some of the other research papers, is included in our protocol. We have used different possibilities of early decoding to enhance the performance of our protocol. We have investigated the performance of with and without early decoding. This investigation is not done so far in the research.

Based on our research work, a paper was published in International Federation for Information Processing (IFIP) / IEEE Wireless Days (WD'10) Conference [18] held in Venice in October 2010.

\subsection{Thesis Organization}

This thesis is organized in the following manner. Chapter 2 is discussing various $\mathrm{NC}$ algorithms and their implementation detail. It also covers some of the benefits of $\mathrm{NC}$ mentioned in the literature. Chapter 3 deals with the application of RLNC to wireless adhoc networks. The main focus is on the algorithms and analytical models proposed so far, dealing with multicasting and broadcasting. This chapter also briefly reviews packet forwarding broadcast protocols. Chapter 4 discusses our proposed model and its implementation detail. Chapter 5 discusses the sensitivity of our protocol as well as the simulation setup. Chapter 6 is related to the performance evaluation and comparison of our protocol to the other selected protocols. Chapter 7 discusses the cross-session performance of our protocol and finally Chapter 8 is related to the conclusion and future work. 


\section{Chapter 2}

\section{Network Coding}

\subsection{Concept}

Network Coding is a relatively new concept in information theory. Unlike the existing store and forward routing schemes, in which data is relayed hop by hop from a source to a destination without being altered, $\mathrm{NC}$ refers to the notion of mixing (linearly combining) information from different flows at intermediate nodes in the network. The receiver decodes these packets to recover the original data when it receives enough coded packets. It has been shown that multicast capacity can be achieved by mixing packets from different flows [3]. As shown in Figure 2.1, each node in a network can perform some computation and output packets are a function of input packets. Intuitively, network coding allows information to be mixed at a node.

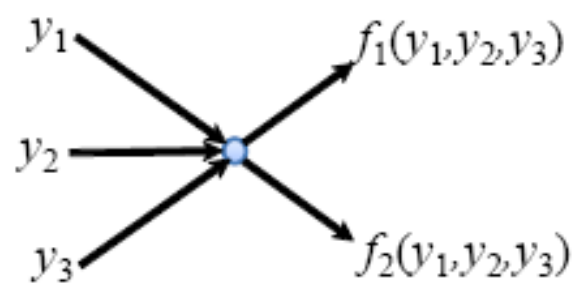

Figure 2.1: Basic NC idea [19] 


\section{Coding Gain}

In terms of $\mathrm{NC}$, coding gain is the effective gain that $\mathrm{NC}$ provides over non-coded packets. The gain can be in terms of Packet Delivery Ratio (PDR), reliability, robustness, number of transmissions or lower end-to-end latency of packets etc.

\subsection{Types of Network Coding}

There are various types of NC that have been applied in the research. NC can be classified into the following three types.

1) XOR-based

2) Reed-Solomon-based

3) Random Linear Network Coding (RLNC)

Each of the above types is explained in detail in the following sections.

\subsubsection{XOR-based NC}

XOR-based algorithms are the simplest algorithm to encode the data packets. The benefit of XOR-based $\mathrm{NC}$ is very well explained in the literature using the famous Butterfly Network for wired networks by Ahlswede et al [3]. The coding gain can be explained as follows:

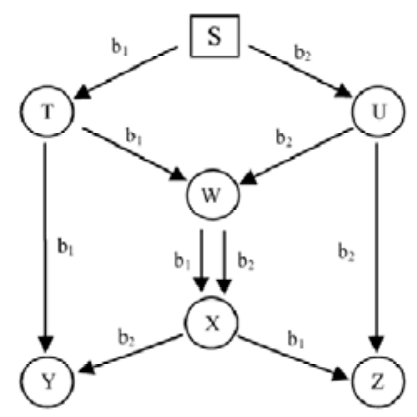

Figure 2.2: Butterfly Network without NC 
Refer to Figure 2.2; it is the case of a butterfly network without NC. The source S wants to multicast two bits $b_{1}$ and $b_{2}$ to two receivers $Y$ and $Z$. Let us assumes that the capacity of each link is 1 bit per second. The source $S$ sends $b_{1}$ through link $S-T$ and $b_{2}$ through link $\mathrm{S}-\mathrm{U}$ as two bits cannot be sent together through the same link at the same time. If we use the standard store and forward scheme, the middle link $\mathrm{W}-\mathrm{X}$ cannot transmit two bits at the same time. W sends the two bits alternately. Now if we calculate the throughput at each receiver, it will not be 2 bits/sec but $1.5 \mathrm{bits} / \mathrm{sec}$ due to the limited capacity of link W - X.

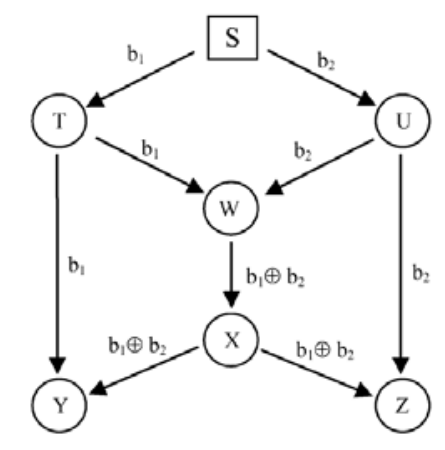

Figure 2.3: Butterfly Network with NC

Refer to Figure 2.3 now, it shows the case of the same network using XOR-based NC. The node $\mathrm{W}$ has enough processing power to linearly combine the two bits it has received by calculating the XOR operation i.e. $b_{1} \oplus b_{2}$. The XOR-ed version is forwarded to node $\mathrm{X}$, which broadcasts the same to the destination nodes $\mathrm{Y}$ and $\mathrm{Z}$. Meanwhile, $\mathrm{Y}$ receives $b_{1}$ from the $T-Y$ link and $Z$ receives $b_{2}$ from the $U-Z$ link.

The decoding is done in a very simple way at the destination nodes. Node $\mathrm{Y}$ is able to decode $b_{2}$ by $b_{2}=b_{1} \oplus\left(b_{1} \oplus b_{2}\right)$. Similarly, node $Z$ is able to decode $b_{1}$ by $b_{1}=b_{2}$ 
$\oplus\left(b_{1} \oplus b_{2}\right)$. If we calculate the throughput, it will be 2 bits/sec. This simple example clearly shows improvement in the throughput of the network using NC.

The slight overhead that can be seen here is the buffer space requirement as well as additional processing in encoding and decoding the packet. With the advancement in solid states like high speed processors and high speed and large capacity memories, these overheads are well taken care of. The bandwidth is limited especially in wireless networks. Using NC, we can efficiently utilize the bandwidth of the network.

The above example is for a wired network. Another example of XOR-based coding, this time in a wireless broadcast network, is provided in the work of Katti et al. [20] and shown in Figure 2.4. They proposed the COPE algorithm, using XOR-based NC. If node A wants to send a message to node B (and vice versa) and there is an intermediate node / router R between them that relays the messages between A and B, the process requires 4 transmissions in total. On the other hand, if $\mathrm{A}$ and $\mathrm{B}$ send their packets to $\mathrm{R}$ and $\mathrm{R}$ broadcasts the XOR version of the packet, a total of 3 transmissions are required. In this case, node A and B can obtain each other's packets by XOR-ing them with their own packets.
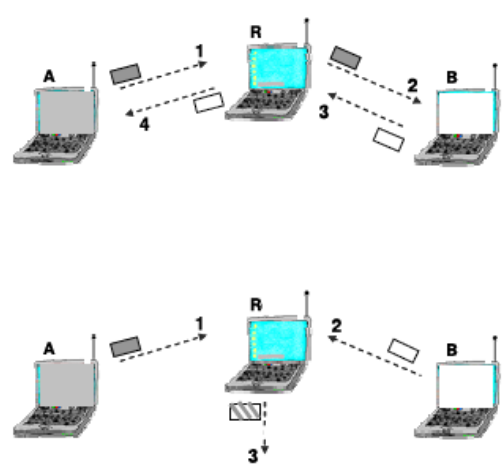

Figure 2.4: Data Forwarding without and with COPE [20] 


\subsubsection{Reed-Solomon-based NC}

Reed-Solomon (RS) codes are block-based error correcting codes with a wide range of applications in digital communications and storage. Reed-Solomon codes are used to correct errors in many systems which include [21]:

- $\quad$ Storage devices (including tape, Compact Disk, DVD, barcodes, etc)

- Wireless or mobile communications (including cellular telephones, microwave links, etc)

- Satellite communications

- Digital Video / DVB

- High-speed modems such as ADSL, xDSL, etc.

A Reed-Solomon code is specified as RS $(n, k)$ with $s$-bit symbols. This means that the encoder takes $k$ data symbols of $s$ bits each and adds parity symbols to generate an $n$ symbol codeword. There are $n-k$ parity symbols of $\mathrm{s}$ bits each. A Reed-Solomon decoder can correct up to $t$ symbols that contain errors in a codeword, where $2 t=n-k$. The following diagram shows a typical Reed-Solomon codeword (this is known as a systematic code because the data is left unchanged and the parity symbols are appended).

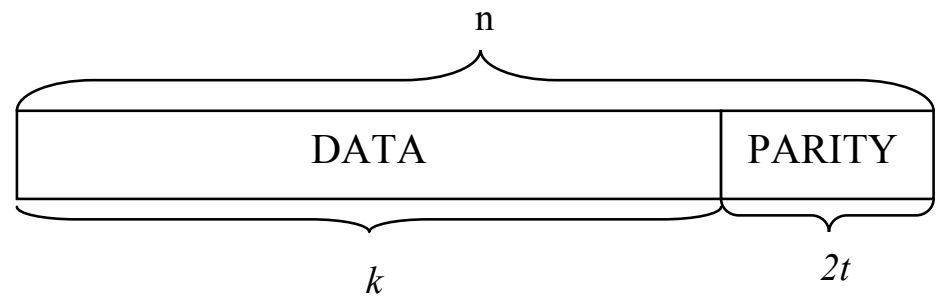

Figure 2.5: RS Codeword 
Example: A popular RS code is RS $(255,223)$ with 8-bit symbols. Each codeword contains $\mathrm{n}=255$ code word bytes, of which $\mathrm{k}=223$ bytes are data and $2 t=n-k=32$ bytes are parity. If the locations of the symbols in error are not known in advance, then a Reed-Solomon code can correct up to $\mathrm{t}=(n-k) / 2$ erroneous symbols, i.e., it can correct half as many errors as there are redundant symbols added to the block. This implies $\mathrm{t}=16$. The decoder can correct any 16 symbol errors in the code word: i.e. errors in up to 16 bytes anywhere in the codeword can be automatically corrected.

Sometimes error locations are known in advance (e.g., "side information" in demodulator signal-to-noise ratios) — these are called erasures. A Reed-Solomon code is able to correct twice as many erasures as errors, and any combination of errors and erasures can be corrected as long as the relation $2 E+S<=n-k$ is satisfied, where $E$ is the number of errors and $S$ is the number of erasures in the block.

RS-code-based NC is used for broadcasting in Mobile AdHoc Networks (MANETS) in [22]. The authors claimed to achieve 61\% coding gain compared to a noncoding approach. The authors defined coding gain as the ratio of the number of transmission required by a specific non-coding approach, to the number of transmissions used by their protocol to deliver the same set of packets to all nodes. However the results are greatly dependent upon the network topology and density of the network. As this protocol extensively relies upon opportunistic listening, sparsely placed nodes do not get much chance of overhearing other messages.

The proposed algorithm works as follows. Let $u$ be the source, $v$ be the receiver where $v \in N(u) . N(u)$ is the set of neighbors of node $u$. Assume that $P$ is the ordered set of $n$ native packets in $u$ 's output queue. Once $u$ broadcasts the coded packets $P$, let $P_{v}$ be 
the set of packets received by node $v$, for each $v \in N(u)$. Let $k=\max \{|P-P v|, v \in$ $N(u)\}$ and $\Theta$ be the $k \times n$ Vandermonde matrix which represents RS codes. Then the minimal number of encoded packets that needs to be sent, such that each neighbor $v$ can decode the packets in $P-P v$ is $k$ and the set of $k$ packets are given by $Q=\Theta \times P$. Therefore a node constructs the coded packet set $Q=\Theta \times P$. It then adds the set of native packet IDs to each coded packet and the index number of codes used. When a node $v$ receives an encoded packet consisting of $n$ native packets $($ set $P), v$ first goes over all native packets received in its packet pool. It collects $P v$, the subset of packets in $P$ that it has already received. It then constructs $\Lambda v$ (the decoding matrix) and adds the new coefficient vector to matrix $\Lambda v$. For each decoded native packet $q$, node $v$ can now process $q$.

\subsubsection{Random Linear Network Coding}

In case of Random Linear Network Coding [23], the output flow at the given node is obtained as a linear combination of its input flows. The coefficients selected for this linear combination are completely random in nature, hence the name Random Linear Network Coding (RLNC). The node combines a number of packets it has received or created into one or several outgoing coded packets.

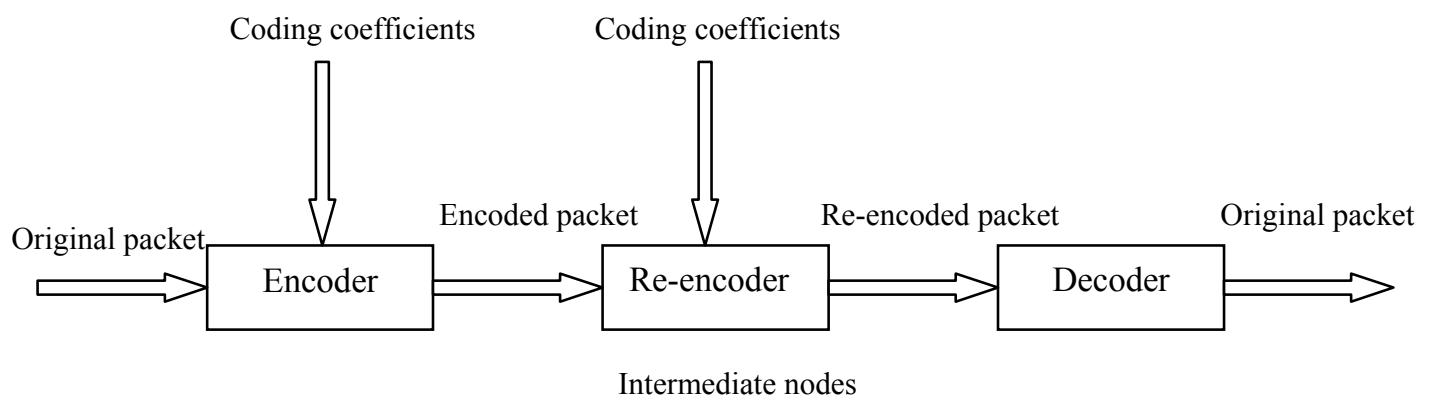

Figure 2.6: RLNC Process 
Typically three different operations are performed by RNLC:

1. Encoding

2. Re-encoding

3. Decoding

The encoding process involves linearly combining the native / original packets with randomly selected coefficients. The coefficients are independently and randomly selected from a finite field called Galois Field (GF). The coefficients of this combination form a coding vector. The encoding, re-encoding and decoding operations are implemented via matrix operations. The re-encoding process is almost similar to the encoding process with the exception that the coding vector of the re-encoded packet is calculated by the arithmetic operation between the newly generated coefficients at that node and the original coefficients of the received coded packets. This simple arithmetic operation can be shown by a simple example.

Suppose a node received two coded packets; $\mathrm{aX}+\mathrm{bX}_{2}+\mathrm{cX}_{3}$ and $\mathrm{dX}_{1}+\mathrm{eX}_{2}+\mathrm{fX}_{3}$. In order to perform the re-encoding operation on the two received coded packets, the node generates 2 coding coefficients $(\mathrm{g}, \mathrm{h})$ for the two coded packets to be re-encoded. The coding vector of the new re-encoded packet can be calculated as following; $g\left(a X_{1}+b X_{2}+c X_{3}\right)+h\left(d X_{1}+e X_{2}+f X_{3}\right)=(g a+h d) X_{1}+(g b+h e) X_{2}+(g c+h f) X_{3}$ where, (ga+hd), $(\mathrm{gb}+\mathrm{he})$ and $(\mathrm{gc}+\mathrm{hf})$ are the new coding coefficients of the re-encoded packet.

The decoding operation is performed at the given node by collecting the coded packets. These packets form a system of linear equations and can be solved forming a 
matrix. The matrix is referred to as decoding matrix. Appendix A contains a detailed description of the encoding, re-encoding and decoding processes.

\section{- Generation}

It is important to limit the size of the matrix that is used for encoding and decoding. For that purpose the packets are grouped together in blocks. Each block is called a Generation. Only packets of the same generation can be encoded and later decoded. It is shown that the size and composition of the generation has significant impact on the performance of network coding [24].

\section{- Dependency}

It is shown that with RLNC, there exists a probability of selecting linearly dependant combinations, which depends upon the size of the GF, i.e., the range of possible coding coefficient values. However, it is shown through simulations that, even choosing a small field size, this probability becomes negligible [8].

\section{- Rank of a Matrix}

The rank of a matrix is the maximum number of independent rows (or the maximum number of independent columns) of a matrix.

\section{- Innovative Packet}

A packet is said to be innovative if it increases the rank of a matrix.

\subsection{Benefits of Network Coding}

Some of the benefits of using NC for wireless networks are mentioned in [2][8]. 


\section{Throughput}

As mentioned before, $\mathrm{NC}$ increases the capacity of a network for multicast flows. It is shown in the literature that, using $\mathrm{NC}$, the same information is delivered while transmitting fewer packets in the network. In case of flooding, the broadcast storm overwhelms the network bandwidth. NC is an effective way to deal with this problem in a distributed way for multicasting and broadcasting [20][23][25].

\section{Reliability}

Some of the main advantages of NC include higher reliability [10] and robustness [14], especially in case of mobile and lossy networks, where other FEC or ARQ schemes do not show good performance. By encoding the packets into a single packet, we are ensuring that a single packet loss does not necessarily require retransmissions [26]. If the complete set of coded packets of the same generation can be received from any node, decoding can be successful and all the packets can be recovered. Similarly, the concept of partial decoding is also provided in the literature where partial packets can still be recovered even if all the required encoded packets are not received.

\section{Distributed Nature}

With NC, there is no need to have global knowledge of the network. Especially in case of RLNC, we even do not care what the neighbour has received. It is highly distributed in nature. Due to this property it is well suited for wireless networks which are also distributed in nature.

\section{Low Complexity}

Overall, $\mathrm{NC}$ works by solving the set of equations linearly combined together in polynomial time. The decoding is performed using Gaussian elimination methods. These 
methods are simple in computation and utilize the cheap computational power to improve network efficiency [16][24].

\section{Mobility}

In mobile environments, the network topology changes over time and a main difficulty for many routing protocols are the frequent route updates and gathering new topological information. NC can address this uncertainty and alleviate the need for exchanging route updates [9][14].

\section{Security}

Sending the linear combination of the packets instead of un-coded packets offers a natural way to take advantage of multipath diversity for security against wiretapping attacks in wireless networks [27]. 


\section{Chapter 3}

\section{Related Work}

\subsection{Broadcast Media}

As mentioned earlier, wireless media, which is broadcast in nature, is a very suitable candidate for NC. As a result, researchers have explored its benefit for wireless networks, especially wireless adhoc networks (both static and mobile). Due to the adhoc nature of the networks, each node is capable of generating as well as routing / relaying the packets in the network from other nodes.

$\mathrm{NC}$ performance is strongly relying on diversity of information, which in the case of wireless networks can significantly improve the performance of multicast and broadcast messages in adhoc networks. The wireless media is unreliable and lossy. There can be frequent retransmissions as required by standard error detection and correction schemes like Forward Error Correction (FEC) and Automatic Repeat reQuest (ARQ). Secondly, in case of broadcasting, reliable broadcast requires that every receiver must receive the correct information sent by the sender. In case of wireless adhoc networks, which are infrastructure-less with limited bandwidth, simple flooding causes bandwidth bottlenecks and loss of packets. Finding more effective ways to multicast and broadcast messages has always been a challenging task and many new protocols and algorithms have been proposed. In this chapter we will focus on the current research trends dealing with efficient broadcasting in multi-hop wireless networks using packet-forwarding approaches as well as using RLNC. 


\subsection{Efficient Packet-Forwarding-Based Wireless Broadcast}

Efficient ways have been proposed in the literature to flood the information within wireless adhoc networks. These broadcasting techniques are categorized [28][29] as Simple Flooding, Probability Based Methods, Area Based Methods and Neighbour Knowledge Methods. For probabilistic flooding, each node retransmits the received packets with probability $\mathrm{P}$. This significantly reduces the broadcast storm problem in simple flooding.

BCAST [30], which is based on the Neighbour Knowledge Method, exchanges periodic HELLO messages to collect 2-hop neighbourhood information. For retransmission, a receiving node A reschedules the packet with random delay if all the neighbours of A are not covered by the previous hop B of the received packet. If the same packet arrives from another neighbour (or set of neighbours) $\mathrm{C}$ who covers the remaining neighbours, A discards the packet. Optimized Link State Routing Protocol (OLSR) [31] and Topology Dissemination Based on Reverse-Path Forwarding (TBRPF) [32] are two additional protocols that implement the Neighbour Knowledge distributed method of dynamically electing a reduced relay set of neighbours for broadcasting information. Each member of the relay set, called Multi Point Relay (MPR), "re-transmits" all the broadcast messages that it receives from its selector node based on certain conditions. These reduced relay set members provide flooding coverage to all 2 hop neighbors from the source. The extension of the above concept of controlled / efficient flooding is applied to the data plane in the Simplified Multicast Forwarding (SMF) algorithm [33]. Other popular Neighbour Knowledge Methods include Dominant Pruning [34] and its improved versions, Total Dominant Pruning and Partial Dominant Pruning [35]. 
The SMF architecture consists of three main components

2) Neighborhood discovery

3) Relay set selection

4) Forwarding process with duplicate packet detection mechanism.

Finding the minimum number of nodes in the Relay set (the forwarding nodes) is an NP-complete problem [34]. There are various relay set selection algorithms proposed in the literature using the concepts of graph theory. In graph theory, a Dominating Set (DS) for a graph $G=(V, E)$ is a subset $V^{\prime}$ of $V$ such that every vertex not in $V^{\prime}$ is joined to at least one member of $\mathrm{V}^{\prime}$ by some edge. $\mathrm{V}$ stands for Vertex and $\mathrm{E}$ stands for Edge in the network. A Connected Dominating Set (CDS) is a DS which is connected.
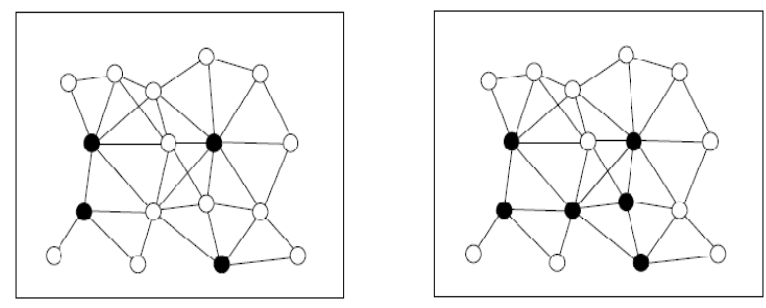

Figure 3.1: Dominating Set (DS) \& Connected Dominating Set (CDS)

CDS-based algorithms are proposed in the literature and their performance is analyzed under high traffic loads and mobility. Among others, one such algorithm is Source based Multi Point Replay (S-MPR), under consideration to be used for SMF.

We compared the performance of our protocol to the simple flooding protocol, a probabilistic flooding protocol, BCAST, and SMF. Simple flooding is chosen as a base line protocol. Simple flooding causes broadcast storm. In order to study the performance of our protocol, we need to compare it with more controlled and efficient flooding 
schemes. The second protocol we selected is probabilistic flooding. Probabilistic flooding is very efficient if the right value of forwarding probability is found and used in the protocol. However such a protocol is not adaptive as we need to find the best value of forwarding probability for every changing scenario for best performance. This value, for example, is very sensitive to the network density. This makes this protocol not practical for adhoc networks. The next protocol we selected for comparison is BCAST. BCAST is adaptive and uses neighbour knowledge to decide if the packets need to be forwarded or not. The PDR and latency performance of this protocol is good for very low data rates of a few kilobits per second (kbps). Finally we selected SMF, which is considered as one of the most efficient broadcast protocols developed based on MPR. Hence we compare our protocol with a wide range of broadcast protocols from baseline flooding to one of the best, namely, SMF.

\subsection{Related Work on RLNC for Wireless Networks}

We divided the related work on RLNC for wireless networks in two categories; Analytical Work and RLNC-based Heuristic Protocols. The details for each category are provided in the following sections.

\subsubsection{Analytical Work}

The original work on network coding for multicasting in wireline networks was done by Ahlswede et al. [3]. They showed that as the symbol size approaches infinity, the source can multicast information at a rate approaching the min-cut between the source and any receiver. The work was further extended by Koetter and Medard [36], showing 
that codes with simple and linear structure were sufficient to achieve capacity in lossless wireline networks. They presented an algebraic framework for network coding, a discipline that is already well established in the mathematical world and proved that there exist coding strategies that provide superior performance without requiring to adapt to the network interior / structure. They derived their results for both delay-free networks and networks with delay.

\subsubsection{NC Performance in Lossless and Lossy Networks}

In [37], the authors gave a theoretical overview of network coding in both lossless and lossy networks for single source unicast \& multicast operation. Their theoretical work shows that, for lossless networks, NC provides no advantage / coding gain in terms of energy efficiency, robustness and reliability compared to standard routing in case of unicast traffic. However, for multicast traffic, NC provides considerable gain. For lossy networks, NC provides coding gain for both unicast as well as multicast traffic. Their results show the benefit of NC especially in providing robustness and reliability in the network. The heuristic implementation of the theoretical work provided in their paper results in a protocol called CodeCast [9], discussed in the next section.

\subsubsection{NC in Distributed Network Operations}

Ho et al. [17] showed that RLNC achieves single source multicast capacity with probability approaching 1 with the length of the code. They demonstrated their results in two scenarios - distributed network operation and networks with dynamically varying connections. They provided a lower bound on the probability of error-free transmission 
for independent or linearly correlated sources. Another important analysis is that RLNC effectively compresses arbitrarily correlated sources in the network in a natural way. The authors compared their distributed NC approach to a Steiner tree routing protocol in their analysis. The results were compared in terms of blocking probability and throughput. The results showed that when the connections vary dynamically, NC can offer significant benefit. Their simulations were based on short network code lengths and networks of 812 nodes. However, the theoretical bound calculated in their work for error-free transmission is considering large field sizes. It is already shown in [38] that choosing even a smaller field size of 8 is enough to make the combination dependency negligible.

\subsubsection{NC Performance in Elastic and Inelastic Networks}

The delay performance of RLNC is studied for elastic and inelastic traffic in [6] for single source broadcast. The authors defined elastic traffic as one with no delay constraints and inelastic traffic as traffic that has stringent delay constraints. Inelastic traffic does not enter the system until the minimum delay constraints are guaranteed to be met. The analytical analysis is done for a single hop system and generalized to multi-hop networks. The results showed that for elastic traffic there is a significant coding gain which is proportional of the file size. For inelastic traffic, it is shown that for the same delay constraints, $\mathrm{NC}$ is able to support a larger number of receivers and improve the throughput of the system. However, in the analysis they assumed Poisson arrival only. Secondly, in order to extend their work to multi-hop scenarios, they rearranged the network in layers and assume that there is no communication between nodes in the same layer for multi-hop networks. Nodes are not allowed to transmit the packets until all the 
nodes in the same layer have received the packets. They did not mention how the layered topology will be constructed and the overhead associated with broadcast messages that will be sent in identifying the layers in which the nodes are to be placed. Finally, their scheme will not be able to function when there is mobility as the nodes might leave the layer or enter another layer's region from time to time.

\subsubsection{NC Comparison with ARQ and FEC Schemes}

In [39], the delay performance of network coding for a tree-based single source multicast problem is studied and compared analytically with various Automatic Repeat reQuest (ARQ) and Forward Error Correcting (FEC) techniques in terms of effective number of retransmissions per packet. For network coding, this paper assumes reliable and instantaneous feedback to acknowledge correct decoding of all data packets. In practical systems, the acknowledgement can be lost as well, especially in highly unreliable wireless networks. The work shows the advantage of coding over ARQ in terms of the expected number of transmissions in a single-path tree or one-hop topology for lossy networks. NC has been shown to be an efficient reliable wireless multicast method which achieves a logarithmic reliability gain over ARQ mechanisms. However, Rateless Coding and link-by-link ARQ achieve comparable performance to that of network coding. However, their analysis ignores the complexity and overhead associated with increasing block size. Although they mentioned that their results show that a reasonable block size is sufficient to obtain the full reliability benefit available via NC, they did not quantify what they mean by sufficient. The other assumption is that each 
node of the multicast tree has exactly $\mathrm{K}$ children, which is not practical, especially when we talk about mobile wireless environments.

The reliability performance of RLNC is compared with two different Automatic Repeat reQuest (ARQ) schemes namely Enhanced ARQ (ARQ-E) and Single Path Routing ARQ (ARQ-SPR) [26]. Reliability is calculated as the total expected number of bits transmitted for each information bit transmitted from sender to receiver. Their results and theoretical analysis show that these advanced ARQ schemes perform comparable to RLNC. The ARQ-SPR scheme gives comparable performance with negligible overhead. However, it is observed in their work that the model they have considered is one with a single sender and a single receiver. We have already discussed that the real benefit of RLNC is observed in the case of multiple unicasts, multicasting, or broadcasting and the papers already discussed before acknowledge this fact. The authors have ignored the coordination and scheduling cost between relay nodes for simplifying the analysis. However, we have observed from our survey that there is no requirement for such coordination in the case of RLNC.

\subsubsection{Energy Efficient Scheme Using NC}

Theoretical analysis is provided as well as simple algorithms are proposed for energy efficient broadcast in [16]. Energy efficiency is directly related to battery life, which is of significant importance in wireless adhoc as well as sensor networks. Their work addresses fixed (topologies and link capacities are not changing) as well as dynamically changing network environments (due to mobility, going to sleep, etc) for allto-all communication patterns, which is our interest as well. Their theoretical analysis 
shows that NC improves performance by a constant factor for fixed networks and by a $\log n$ factor for dynamically changing networks, where $\mathrm{n}$ is the number of nodes. Their assumption in the system model is that a broadcast transmission is successfully received by all neighbours or the complete transmission will fail. Secondly, each source has only a single packet to transmit. The paper also described issues related to generation selection and management based on multi-source scenarios. Although the authors have hinted at these generation management methods, no detail is provided as to how the generation management is actually working. Secondly, in their work, they have assumed that each node has only a single symbol to transmit and all the packets belong to a single generation. This assumption is not practical in a sense that there will always be multiple packets to be transmitted by sources and if the number of nodes increases, keeping all the packets in a single generation will not be practical in terms of memory utilization and processing time.

\subsubsection{Improvement of Distributed MAC Protocol using NC}

In [25], the authors provided an extension to distributed MAC protocols that improves efficiency of coding decisions and allows decodability of packets before they are transmitted. They provided an algorithm (NC-MAC) that manages the stored data packets intelligently at the MAC queue of each node. The algorithm improves the knowledge of the node for available correct coding opportunities by using opportunistic acknowledgements. They showed that their protocol shows significant throughput improvement compared to standard NC. However this work is related to XOR-based NC, which works on the neighborhood knowledge of received packets. 
Similar work is done for the case of unicast traffic [40], showing a $20-30 \%$ throughput increase using their RLNC-based proposed algorithm, named Multipath Code Casting $\left(M C^{2}\right)$. Another paper shows almost two-fold throughput increase [41] compared to traditional routing when their RLNC-based algorithm is applied, named Optimized Multipath Network Coding (OMNC).

\subsubsection{RLNC-based Heuristic Protocols}

In this section we discuss in detail the various RLNC-based routing algorithms proposed and their analysis. Later, we sum up the performance of RLNC-based protocols mentioned in the literature, thus developing the basis for our proposed algorithm.

\subsubsection{RLNC-based Probabilistic Routing}

The first protocol [14] is related to multisource unicast for wireless adhoc networks using RLNC for probabilistic routing (Delay Tolerant Networking) in extreme performance-challenging environments. The simulation results show that their proposed RLNC-based probabilistic routing algorithm achieves high reliability and robustness compared to a simple probabilistic routing scheme for both static and mobile nodes. Hashing is performed over the sender address and packet identifier to determine which generation the packet should belong to. However the paper did not provide much detail on this hashing operation. In order to forward the packets, a forwarding factor $d$ is introduced. The results for a static topology show that NC achieves $100 \%$ delivery ratio with less overhead, whereas probabilistic routing results in a three times larger overhead to achieve the same PDR. For the mobile topology, the authors claim similar results, 
$100 \%$ packet delivery ration is achieved with a forwarding factor of 0.125 , resulting in 6 times overhead reduction compared to simple probabilistic routing. For sparse networks, NC performs much better and probabilistic routing almost fails to deliver the packets. They also investigated the impact of generation size on the overhead and size of matrix. For a generation size of $4,99 \%$ of PDR is achieved. The results also confirm that an increase in the generation size improves the network throughput as well as delivery ratio. However, even a smaller generation size is shown to perform well compared to simple probabilistic routing.

\subsubsection{RLNC-based Video Surveillance Protocol}

CodeCast [9] is proposed for multimedia applications, especially for surveillance i.e. for transmitting video images collected from various cameras to the patrolling security agents in an industrial environment. The main focus is on delay constraints and delivery ratio for single source wireless multicast. The images should be delivered successfully within the delay constraints.

The authors used the term "Block" in their research work in lieu of "Generation" used in the literature and in our thesis. The application generates equal-sized frames $\mathrm{p} 1$, p2 ....Adjacent frames are arranged into blocks denoted by (blockid, blocksize). The blocksize (\# of frames to be encoded) is kept variable based on the delay constraints calculated from the frame generation rate of the application. However their results are presented for only two distinct block sizes, 4 and 8 . The end-to-end delay increases when the blocksize is increased to 8 as more frames are needed from the application to encode them. However, increasing blocksize (generation size) improves the network throughput, 
as also mentioned in other literature. The receiving node has a timer called blocktimeout. Based on the value of blocktimeout, the node makes the forwarding decision. If all the packets of a particular block are received, then they are decoded and recovered. However, as mentioned in [15], some of the packets can be recovered even if fewer than blocklength packets are received if the rank of the sub matrix is full. However, this paper did not talk about decoding based on partially received packets. The paper compares the performance of CodeCast with the On Demand Multicast Routing Protocol (ODMRP), which is claimed to be one of the best multicast routing protocols in mobile lossy environments. The results show $100 \%$ packet delivery regardless of node speed, block size and packet drop probability compared to 94\% for ODMRP, with less overhead.

\subsubsection{RLNC-based Broadcast in Realistic Simulation Scenarios}

In [15], the authors observed the effect of packet loss and propagation delay using RLNC. Their work addresses single-source broadcast in wireless adhoc networks. They showed through simulations that network node density and generation size play an important role in the performance of RLNC-based broadcast. The encoding, re-encoding as well as decoding processes are almost similar to that of CodeCast with minute differences. It is unclear in the paper how the re-encoded packet's rank is identified.

The performance metrics used to evaluate the broadcast schemes are delay, packet loss rate, protocol overhead and transmission fairness. The results show that as the network becomes more crowded, NC loses more packets with neighborhood size > 14. The reason given is that, in case of dense networks, there is a higher chance that all packets are received and the simple broadcast scheme works well. However they did not 
mention the fact that in case of dense networks, there are more collisions, and packet loss due to collisions should increase with denser network, thus causing broadcast storm. Secondly, the results from other references show that in denser networks RLNC shows better performance. Another important factor is that they have considered only static scenarios. As described before, much improved performance is achieved for lossy as well as mobile networks compared to standard broadcast. The protocol overhead for RLNC decreases when a larger generation size is used, but they did not mention the fact that once the generation size increases, it affects the delay performance of the algorithm. As mentioned in previous work, the balance between the delay constraint and generation size need to be established. Their work lacks this analysis.

\subsubsection{RLNC-based Broadcast in Dense Environment}

Broadcasting with RLNC for dense wireless environment is presented in [42]. The work is for single-source adhoc networks. In their proposed algorithm, the source node divides the information into groups of $\mathrm{N}$ packets and every packet in the same group is assigned the same sequence number. If a receiving node finds the sequence number of the arriving packet to be one that has already been recovered, the node discards that packet. In simulations, the authors have made the assumption that there is no buffer overflow and no bit error packet loss. Secondly, decoding failure causes all packets to be lost. They did not look at the possibility of earlier decoding of packets as mentioned in [15]. The metric used to judge the performance is decoding failure, packet loss probability vs. node density, vs. length of coding vector $\mathrm{N}$ and vs. order of GF. Their results show that as the length of the coding vector increases, the number of collided packets decreases. 
Secondly, as the node density increases, $P_{\text {loss }}$ becomes a convex function. As the number of nodes increases, there are more chances to successfully decode the packets, but on the other hand a conflicting situation arises as there are more collisions and the chances of a decoding failure increases as well. Their $P_{\text {loss }}$ results are also in conflict with the results mentioned in [15], who showed that RLNC shows poor performance when the number of nodes increases. Another result between $P_{\text {loss }}$ and length of coding vector shows that there is strong influence of the size of the coding vector and $P_{\text {loss }}$. Choosing the optimal vector size is necessary for better $P_{\text {loss }}$ performance. Similarly, they have shown that the order of the GF field also has a strong impact on $P_{\text {loss. }}$ The authors did not analyze the performance of their algorithm in case of mobility and lossy environments.

\subsubsection{RLNC-based Wireless Broadcast for Multi-player Video Game}

Finally, multisource wireless broadcast using RLNC is discussed in [43]. The algorithm is developed for multi-player video game broadcast for wireless networks called Network Coded Piggy-Back (NCPB). The proposed algorithm is compared with IEEE 802.11 broadcast, Piggy-Back Retransmission (PBR) and Multi Point Relay (MPR) in terms of packet delivery ratio and delay. The simulations were carried out for lossy static as well as mobile scenarios involving multiple sources. However their work is more specific to the gaming environment where there is a periodic nature of traffic.

Before starting the game, the $\mathrm{N}$ nodes negotiate with each other for entry into and initialization of the game. Each node obtains an ID and their coding vectors are exchanged among each other during the initialization phase. Afterwards, each node uses the same coding vector. The authors did not describe the impact of linearly dependent 
coding vectors. All the coding vectors generated and exchanged should be checked for dependency. Another point, as mentioned in [16], is that each source packet should only be part of one generation but the authors in this paper did not mention anything related to dealing with this issue or how they are creating generations. The exchanges take place in each time interval, which are well synchronized between nodes. However, in case of adhoc networks, an algorithm should be designed that does not require or depend upon synchronized nodes. This algorithm showed high delivery ratio and less delay suitable for gaming in all simulations compared to other schemes. Their results also show that once the node density increases, the performance degrades for other schemes, but the NCPBbased scheme shows better results. The same performance is observed when conducting experiments on their test-bed implementation.

\subsection{Discussion}

As we have seen from the analytical work as well as various proposed protocols for RLNC, there is a strong potential of using network coding for various applications. The work on wireless networks shows many promising results. Based on the survey, we conclude the following for the application of RLNC for wireless applications.

1. RLNC is very suitable for multicast / broadcast applications.

2. The analytical models show the benefit of RLNC in terms of reliability, robustness throughput and energy efficiency. However, all these models are based on certain assumption that may limit their analysis in the context of real applications.

3. RLNC shows almost similar performance compared to advanced ARQ and other controlled flooding schemes in case of static and low density networks. In harsh 
environments, especially where there is sparse connectivity and lossy links, RLNC performs better than other counterpart schemes.

4. For dense networks, there are more collisions and hence more retransmissions. RLNC is shown to provide better results compared to other schemes even though there are losses due to collisions.

5. In case of mobile networks like MANETs, RLNC performs better and provides more robustness and reliability with less overhead compared to other schemes

6. The performance of RLNC is very much dependent upon the generation size, size of GF (symbol size) and how the extra (redundant) transmissions are controlled in case of broadcasting.

7. Increasing the generation size improves the throughput, but there is a strong relation between the decoding complexity, delay and selection of a proper generation size.

8. Another important issue is defining the role of intermediate nodes (sub-graph selection problem). Different papers have proposed various methods to improve the delivery ratio as well as throughput by storing the coded packets at intermediate nodes and either decode, partially decode or re-encode the received encoded packets. An intermediate node also needs to decide whether to send multiple copies or a single encoded packet.

9. Most schemes have parameters to control the number of retransmissions. However, these parameters are manually set for each scenario and therefore are not adaptive, which makes these protocols not suitable for different node densities and speeds. A suitable algorithm needs to be developed that can take care of all the possibilities and 
provide the most effective solution for a wide range of dynamically changing environments.

10. Most of the work in wireless networks is either related to multiple unicast or multicast scenarios; there is less work done investigating the performance of broadcasting in adhoc networks using RLNC. There are analytical models as well as a few simulation papers that discuss broadcast, but there is still a lot of room to develop an algorithm that is suitable and practical in implementation. Especially very little research has been done to-date for multi-source broadcast.

11. The problem dealing with multi-source broadcast has to be handled quite differently from the proposed schemes for a single source. The main issue is how the generation is to be defined for packets from different sources. Some of the proposals for selecting a generation include packets generated within a specific area of network, packets generated over a specific period of time or packets containing a certain type of information.

12. It is mentioned in the literature [42] that for multi-source broadcast, the initial assumption is that all the nodes are well synchronized. However our protocol is totally distributed in nature and does not require node synchronization.

13. To the best of our knowledge, there has been no comparison of the performance of multi-source wireless broadcast with and without cross-session generations in the literature. The cross-session generation concept is explained in more detail in the next chapter. 


\section{Chapter 4}

\section{Proposed Model}

\subsection{Adaptive Random Linear Network Coding with Controlled Forwarding (ARLNCCF)}

Based on the analysis from Chapter 3, we propose Adaptive Random Linear Network Coding with Controlled Forwarding (ARLNCCF) for broadcasting in wireless adhoc networks. The proposed algorithm is carefully designed based on the concepts and shortcomings of previously proposed algorithms. Our main target is to present a single algorithm that can meet the needs of various environments and situations and is not limited to a single scenario, hence the reason we call our protocol adaptive. This chapter introduces our approach and our algorithm's functionality in detail.

\subsection{Proposed Scheme}

RLNC is highly distributed in nature. Unlike XOR-based or Reed-Solomon-based coding, which require the knowledge of what its neighbours have received to encode the packets, no such information is required by RLNC. Broadcasting is an important communication technique and needs the same attention as unicast and multicast. Most of the work found in the literature is addressing the case of single source broadcasting. There are few proposed adaptive algorithm dealing with multi-source broadcasting. Our algorithm will be suitable for both single source and multi-source broadcasting. It is observed from the previous work that RLNC works well for dense, mobile and lossy 
environments where other algorithms show poor performance [42]. However, if the node density is small, its performance depends upon how many packets are encoded together to obtain a better coding gain [14]. ARLNCCF is able to combine packets from the same source or multiple sources based on available generations and their sizes in the buffer.

Our protocol follows the basic idea of RLNC as discussed in Chapter 2. We got the inspiration from [9][15][16] to develop our protocol. It is mentioned in the literature $[38][44]$ that $\operatorname{GF}\left(2^{8}\right)$ is sufficient for the symbol size to maintain linear independence with high probability. So for simplicity and byte-by-byte operation, we set the symbol size to 8 in our implementation. Some of the terms used are as following,

\section{Coded packet}

Once the source generates the packet, it is encoded with a random coefficient from $\mathrm{GF}\left(2^{8}\right)$, the resultant packet is called coded packet. This packet is re-encoded with other coded packets in the generation (if they exist) before transmission.

\section{Re-encoded packet}

When the existing coded packets are further encoded with random coefficients from $\mathrm{GF}\left(2^{8}\right)$, the resultant packet is called a re-encoded packet.

\section{Decoding matrix}

All the packets are stored locally in generations in the form of a decoding matrix. Each row of the matrix contains the coefficients of the coded/re-encoded packet.

\section{Coded vector}

The vector of coefficients that are stored as rows in the decoding-matrix for each encoded/re-encoded packet is called coded vector for that packet. 


\section{Cross-session generations}

Generations that are not confined to particular sources (generations having symbols from the same source only) and allow inter-mixing of symbols from different sources are called cross-session generations. In this thesis we show that cross-session generations improve PDR and reduce latency compared to generations that do not allow symbols to be combined from different sources (see results in Section 7.1)

The unique features of our protocol that make it adaptive and support crosssession design are discussed in the following sections.

\subsubsection{Hello Control Messages and Number of Retransmissions}

In order to adapt the number of retransmissions as well as the probability of broadcast, we need to maintain the neighborhood information for the topology. Based on this neighborhood information, the algorithm can decide about the node density as well as the number of retransmissions required.

Each node sends Hello messages periodically with its own neighbourhood information stored in the Hello packet. In this way each node can obtain the two-hop neighbourhood information. If we assume node " $\mathrm{m}$ " as a starting point, the set of neighbours of $\mathrm{m}$ is given by $\mathrm{Nr}(\mathrm{m})$ and the neighbours of neighbours of $\mathrm{m}$ are given by $\mathrm{Nr}_{\mathrm{N} 1}(\mathrm{~m}), \mathrm{Nr}_{\mathrm{N} 2}(\mathrm{~m}) \ldots \mathrm{Nr}_{\mathrm{Nn}}(\mathrm{m})$, where, $\mathrm{Nr}_{\mathrm{Nn}}(\mathrm{m})$ is the set of neighbours of the $\mathrm{n}^{\text {th }}$ neighbour of $\mathrm{m}$. As per Figure 4.1, the neighbours of $\mathrm{m}$ and the neighbours of neighbours of $\mathrm{m}$ are as follows: 
$\mathrm{Nr}(\mathrm{m}):\{\mathrm{N} 1, \mathrm{~N} 2, \mathrm{~N} 3\}$

$\mathrm{Nr}_{\mathrm{N} 1}(\mathrm{~m}):\{\mathrm{m}, \mathrm{N} 8\}$

$\mathrm{Nr}_{\mathrm{N} 2}(\mathrm{~m}):\{\mathrm{m}, \mathrm{N} 6, \mathrm{~N} 7\}$

$\mathrm{Nr}_{\mathrm{N} 3}(\mathrm{~m}):\{\mathrm{m}, \mathrm{N} 4, \mathrm{~N} 5\}$

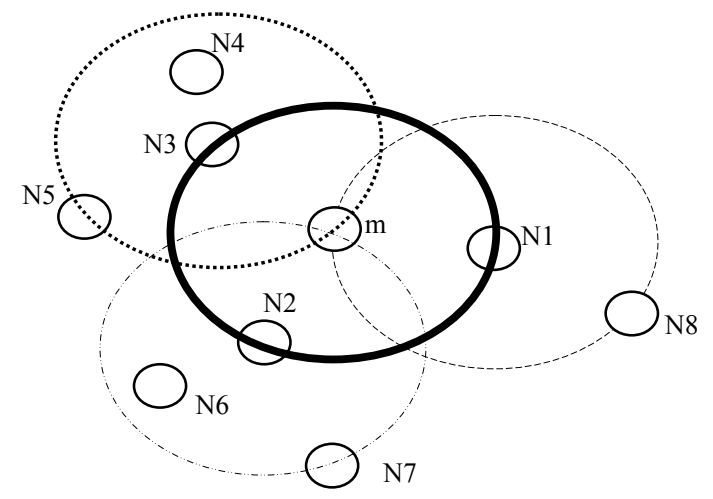

Figure 4.1: Neighborhood of Node m

Based on the neighbour's neighbour information, the node will compute the neighbouring node with the minimum number of neighbours, i.e. $\operatorname{Min}\left(\mathbf{N r}_{\mathrm{Nn}}(\boldsymbol{m})\right.$, for all n). It will compute $\mathrm{N}_{\mathrm{T}}(\mathrm{i})$, the number of transmission required for that generation as follows:

$$
N_{T}(i)=\text { Igeneration size } / \operatorname{Min}\left(N r_{N n}(m), \text { for all } n\right) 7
$$

The node will transmit $N_{T}$ (i) packets for that generation. In case of a dense network, not every node needs to retransmit coded packets. So for dense networks, where the ratio is $<1, \mathrm{~N}_{\mathrm{T}}$ will become the probability to rebroadcast We don't use the ceiling function in this case. So if the ratio is $<1$,

$$
P_{T}=\text { generation size / } \operatorname{Min}\left(N r_{N n}(m), \text { for all } n\right)
$$

The rationale behind this formula is that each node is guaranteed to receive at least generation size coded packets, thus allowing the node to decode all original packets.

\subsubsection{Packet Format}

We define our own packet format for ARLNCCF. Finding space in the IP header is quite challenging due to very limited space availability. All previous works related to RLNC have defined their own packet format. We have also defined our own packet 
header format where the required information will be stored. In the header, each coded symbol needs to be identified in the encoded vector attached to the packet. We identify each symbol with a Sequence Number (16 bit) and IP address (32 bit) pair. The header fields are shown in Figure 4.2.

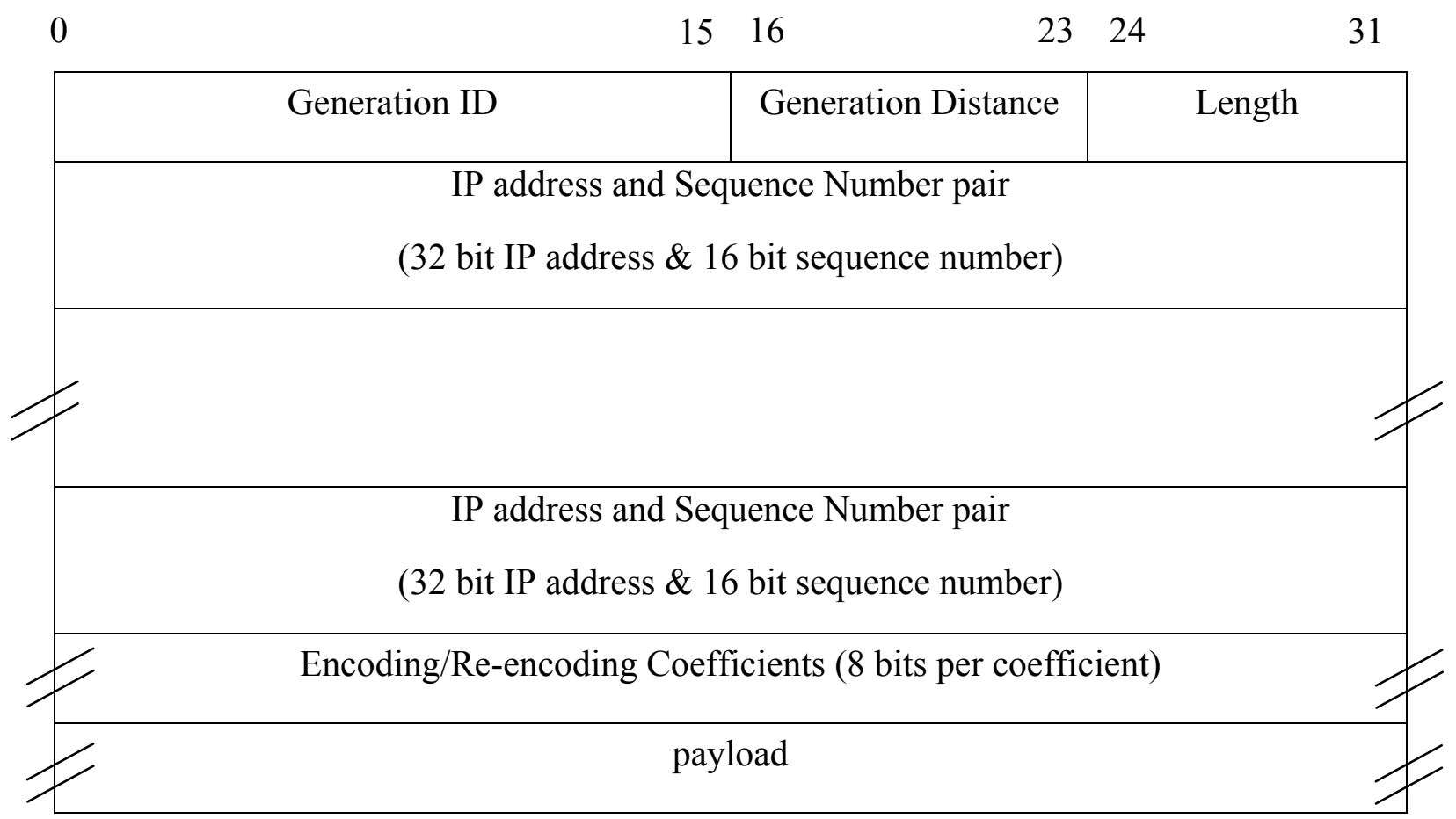

Figure 4.2: Packet Format for ARLNCCF

The Generation ID is the 16 bit number used to represent each generation uniquely at the given period of time. Generation Distance is an 8 bit number and is explained in detail in Section 4.2.5. The length field specifies how many source address and sequence number pairs we have. The IP address and Sequence Number pair is used to uniquely identify the original packet in the encoded vector. We have as many pairs as that of number of original packets encoded together. This pair is required because if we just 
use one parameter than there is no way to distinguish packets from one source to another. Each source maintains its own sequence number and each packet can only be distinguished by its sequence number and the source address generating that sequence number. Similarly, we have as many coding/ re-encoding coefficients (encoding vector) as that of source address and sequence number pairs. This encoding vector is inserted in the respective Generation as the last row in the decoding matrix by the receiving node. Finally we have the payload part which contains the actual coded packet.

In order to implement our protocol in real world, we need a new protocol value in the IP header's "Protocol" field. This value is assigned by the Internet Assigned Numbers Authority (IANA). An ARLNCCF packet is carried as the payload of an IP packet. At layer 3, once the IP header's protocol field is examined with our protocol value, the packet will be send to the ARLNCCF protocol implementation for further processing.

\subsubsection{Generation Size}

Since our main aim is to develop a multi-source protocol and nodes are free to insert their packets in any generation, there will always be cases where different nodes insert their symbols in the same slot of a given generation based on their local space in that generation. The receiving node maintains an ordered list of source addresses and sequence numbers for each locally saved generation. Once a coded packet arrives, the node reorders the symbols of the receiving packet based on its local ordered list. If a symbol is found with a different 2-tuple for the same slot, this symbol is moved to the available space in that generation. If no space is available, the generation size is increased by 1 and the conflicting symbol is added to the end. 


\subsubsection{Generation Timeout}

Motivated by the generation timer concept introduced in [9][15], our protocol also has a timer $\mathrm{T}$ associated with each generation. The required number of encoded packets is rebroadcasted after the timer expires. However, there is still a chance that the node receives more innovative packets after $\mathrm{T}$ has expired. In that case, a single packet is rebroadcast for each received innovative packet if $\mathrm{N}_{\mathrm{T}}>1$.

\subsubsection{Generation Distance (GD)}

In order to control the generation size and to avoid increasing it by a large value, especially at high data rates and a large number of senders, we introduce the idea of a generation distance. It works as follows:

1. The source, creating the new generation, sets the generation distance to 0 for that generation. The rebroadcasted packets for that generation have this value set to 1 in the packet header.

2. When the node receives a packet, it compares the generation distance value for that generation with the value in the packet. If the packet value is less than the locally stored value, the locally saved value is replaced with the packet value. In this way the minimum hop distance is known to the node from where the generation was created.

3. If the source inserts its packet in another generation, not created locally, the value remains unchanged and the re-encoded packet for that generation has the value incremented by 1 .

4. To insert a new packet, locally created generations are preferred. If none are available, then the generation with minimum GD is preferred, as long as the GD value 
is less than or equal to a given threshold. If all the available generations have a value above that threshold, a new generation is created.

The whole process can be explained with a flow diagram as shown in Figure 4.3.

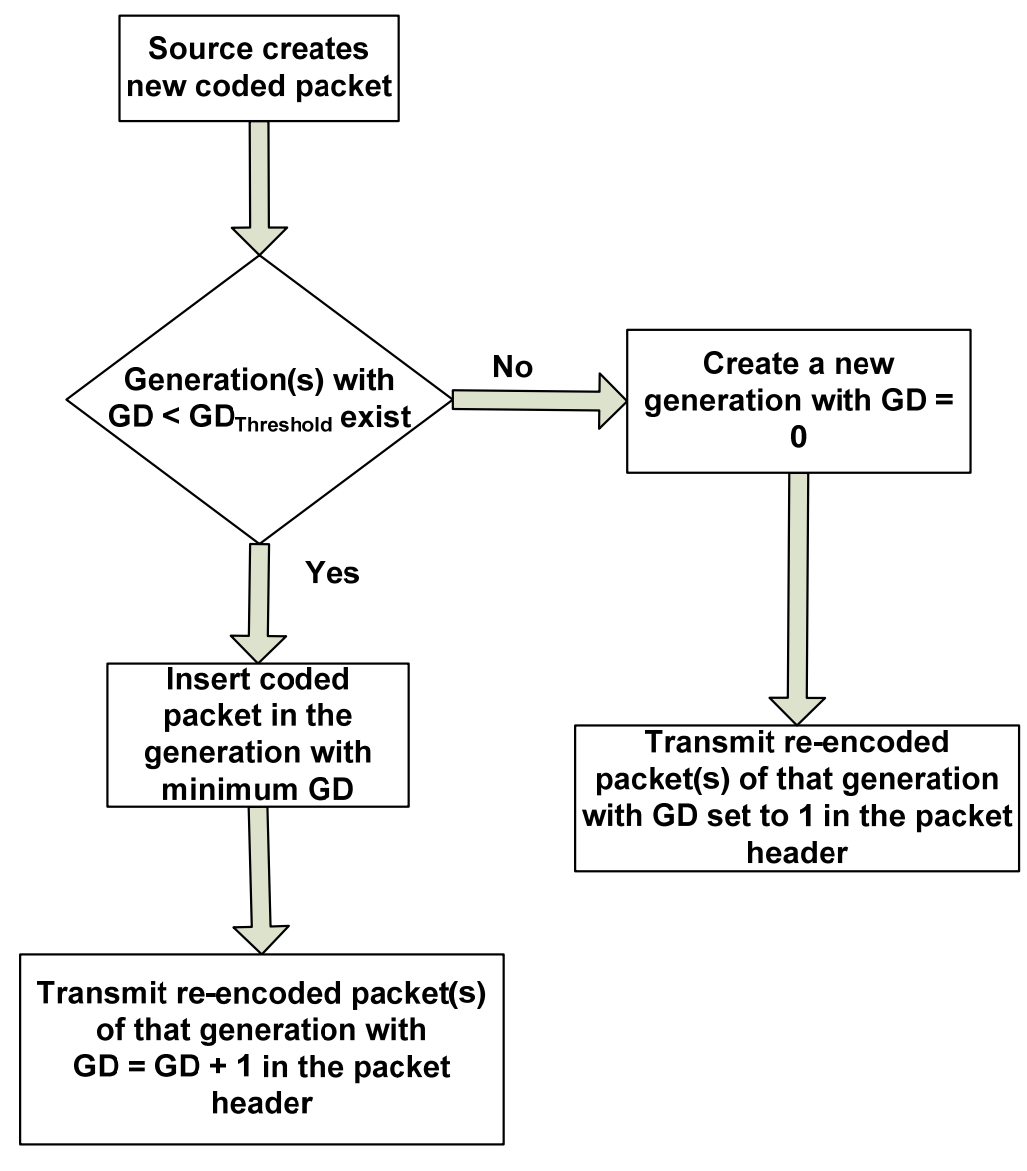

Figure 4.3: Flow Diagram of GD Concept

\subsubsection{Partial and Full Decoding}

Decoding is done by the Gauss-Jordan elimination method [45]. Since source packets are re-encoded with other packets already in the generation, there will always be a possibility for each node to partially decode the generation. The generation is partially decoded once the rank of the sub-matrix is full. All the packets that are decoded for that generation are recorded to prevent passing duplicate packets to the upper layers. 


\subsubsection{Generation ID -Duplication}

The Generation ID is randomly generated by the source node and it should be unique in the network. According to [44], one or two bytes are enough to be reserved for the generation ID in the encoded packet. In our simulation, we will limit it to 2 bytes. To further reduce the probability of duplicate ID's in the network, each node maintains the list of all IDs seen so far in a given frame of time. If the node generates a new ID, it will be checked against the list. If that ID is already in the list, a new ID is generated again till it is not present in the list. The list is periodically refreshed.

\subsection{Operation of ARLNCCF}

Our focus is on a multi-source broadcast environment where every node is a receiver. The source node performs multiple operations depending upon certain conditions. The whole process is explained below.

\section{Notations:}

\begin{tabular}{|c|c|}
\hline Symbol & Meaning \\
\hline $\mathrm{G}(\mathrm{i})$ & $\mathrm{i}^{\text {th }}$ generation \\
\hline $\mathrm{GD}(\mathrm{i})$ & Generation distance of $\mathrm{i}^{\text {th }}$ generation \\
\hline $\mathrm{T}$ & Timer for generation \\
\hline $\mathrm{T}(\mathrm{i})$ & Timer for $\mathrm{i}^{\text {th }}$ generation \\
\hline $\mathrm{N}_{\mathrm{T}}(\mathrm{i})$ & \# of transmissions for $\mathrm{i}^{\text {th }}$ generation \\
\hline $\mathrm{GD}_{\text {Threshold }}$ & Generation distance threshold \\
\hline $\mathrm{Nr}_{\mathrm{Nn}}(\mathrm{m})$ & Neighborhood of $\mathrm{N}_{\mathrm{n}}^{\text {th }}$ neighbor of node $\mathrm{m}$ \\
\hline $\mathrm{Nr}(\mathrm{m})$ & Neighborhood of node $\mathrm{m}$ \\
\hline
\end{tabular}

Table 4.1: Notations used in the Algorithm 


\subsubsection{Source Node Operation}

1. The source generates a packet. The packet consists of multiple symbols of 8 bit each. If we assume an IP packet length of 1400 bytes, there will be (up to) 1400 symbols in each packet, considering GF $\left(2^{8}\right)$. The encoding operation is performed on each packet.

2. Once the packet is generated, the node checks if there are already some generations available in the memory with GD $<\mathrm{GD}_{\text {Threshold. }}$

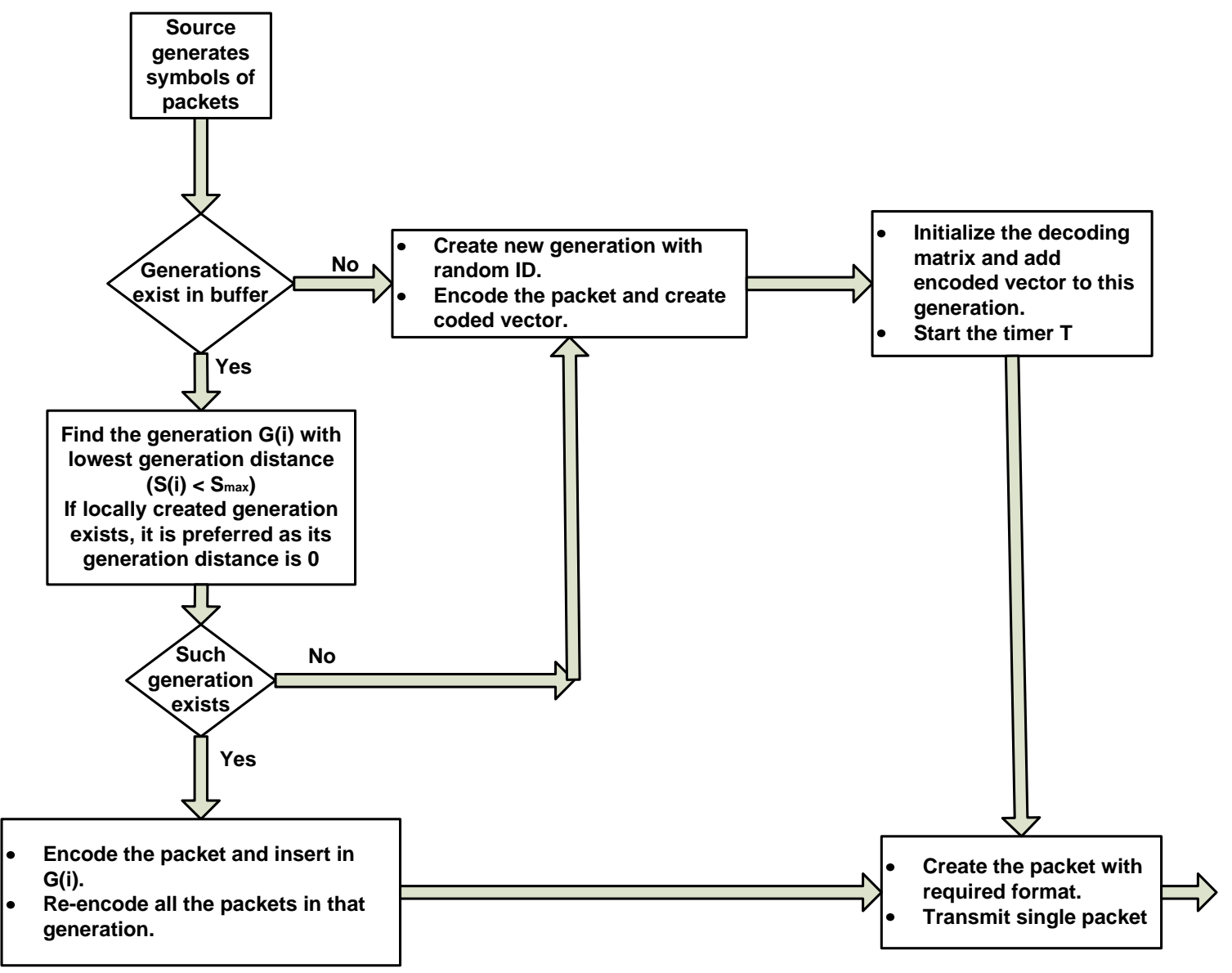

Figure 4.4: Flow Diagram - Encoding Process 
3. If YES (Generations exist in the memory):

a. Find the generation G(i) in memory with lowest generation distance, i.e. min $\mathrm{GD}(\mathrm{i})$. If there are more than one generations with same minimum GD value, than the first generation found by the algorithm with that GD value is used.

b. Generate a random coefficient from $\mathrm{GF}\left(2^{8}\right)$ for that packet and insert the packet in the generation G(i).

c. Re-encode all the available coded vectors including the source vector in the decoding matrix. A single coded packet is broadcasted.

4. If NO (No suitable generation is available in memory):

a. A new generation is created with randomly selected generation ID. The generation is saved in the memory in the form of a decoding matrix.

b. The coded vector is created by choosing random coding coefficients.

c. An encoded packet is created with the header containing the generation ID and coding coefficients.

d. A single encoded packet is broadcasted, as this generation contains one new source packet.

\section{Example: Source packet insertion}

Source $S 1$ has a packet $m 4$ to send. The packet is encoded with random coefficient, $x 3=g 5 * m 4$, where $g 5$ is taken from GF $\left(2^{8}\right)$. If no generation is available then a new generation is created and $x 3$ is inserted in the new generation and transmitted (there is no re-encoding in this case). We assume that the source already has two coded packets in the some generation in the memory, $g 1 * m 1+g 2 * m 2$ and $g 3 * m 1+g 4 *$ m3. The two coded packets contain symbols from 3 original packets, $m 1, m 2$ and $m 3$. 
The source decides to put its packet in this generation. The source packet is encoded and $x 3$ is added to this decoding matrix. Now the generation has symbols from 4 original packets. The source re-encodes all these packets into a single re-encoded packet and broadcasts with $100 \%$ probability as this generation includes a new source packet.

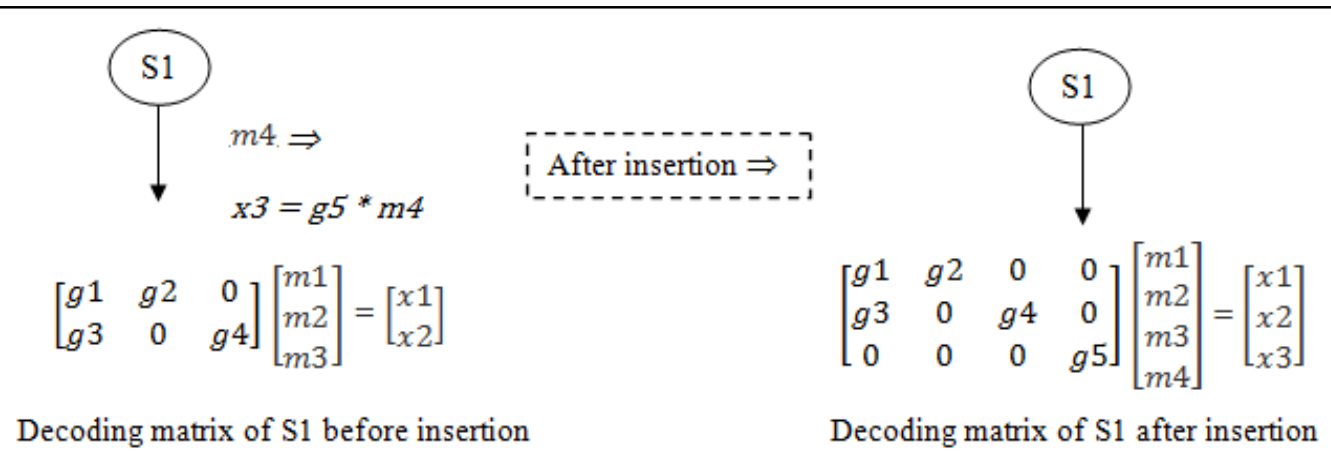

Figure 4.5: Single Packet Insertion

In order to transmit the re-encoded packet, the source will generate 3 random coefficients; assume these are $g 6, g 7$ and $g 8$. It will linearly combine the coded packets and create a single re-encoded packet as following.

$$
\begin{aligned}
& g 6(g 1 m 1+g 2 m 2)+g 7(g 3 m 1+g 4 m 3)+g 8(g 5 m 4) \\
& =(g 6 g 1+g 7 g 3) m 1+(g 6 g 2) m 2+(g 7 g 4) m 3+(g 8 g 5) m 4
\end{aligned}
$$

The new packet will look like the following,

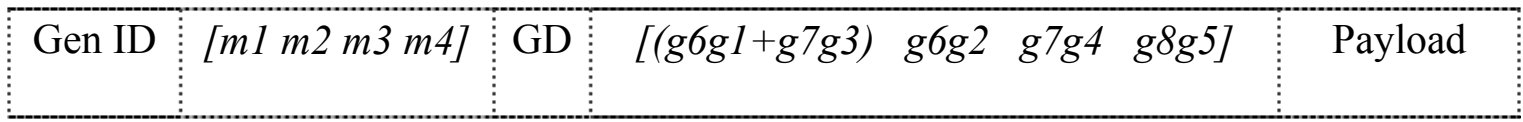

\subsubsection{Intermediate Node Operation}

The node which is not the source is referred here as intermediate node. The intermediate node operation is explained with the flow diagram shown in Figure 4.6. 
1. The node receives an encoded packet. The memory is checked to see whether the generation of the received packet already exists in the memory.

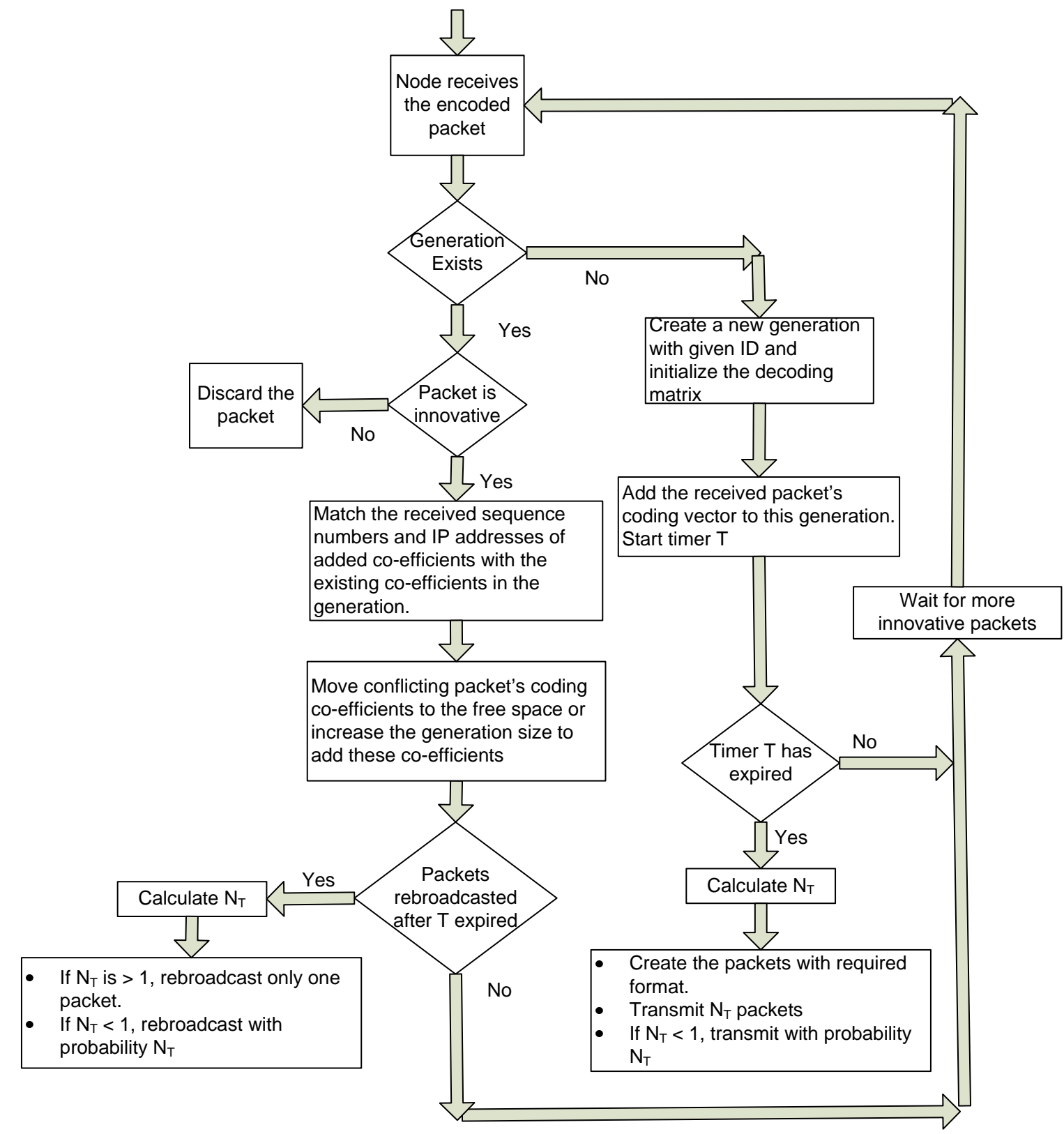

Figure 4.6: Flow Diagram - Intermediate Node Operation

\section{If NO (Generation does not exist in memory),}


a. The nodes create a new generation with the generation ID taken from the received packet and the packet is inserted in the generation in the form of a decoding matrix. Timer $\mathrm{T}$ for the generation is started.

b. If the timer $\mathrm{T}$ has expired, calculate $\mathrm{N}_{\mathrm{T}}$ and create $\mathrm{N}_{\mathrm{T}}$ packets with required format. Transmit $\mathrm{N}_{\mathrm{T}}$ packets. If $\mathrm{N}_{\mathrm{T}}<1$, transmit with probability $\mathrm{N}_{\mathrm{T}}$.

c. It the timer $\mathrm{T}$ has not expired, continue waiting for more innovative packets.

3. If YES (generation exists in memory),

a. The packet is checked if it is innovative. If the packet is not innovative, it is discarded. If the packet is innovative, the sequence number and IP addresses of received symbols are matched with existing symbols in the generation.

b. There will be a conflict in the packet's coefficients when a packet with different sequence number and IP address pair is found in the slot, compared to the locally stored packets for that generation.

c. In case of conflict, move the conflicting coefficients in the received packet to the free space in the generation. If there is no free space available, increase the generation size by one and move the conflicting packet's coefficients to that location.

d. In case $\mathrm{N}_{\mathrm{T}}$ packets are already re-broadcasted after $\mathrm{T}$ has expired, calculate $\mathrm{N}_{\mathrm{T}}$ again.

e. If $\mathrm{N}_{\mathrm{T}}>1$, transmit only one packet. Otherwise transmit packet with probability $\mathrm{N}_{\mathrm{T}}$.

f. If timer $\mathrm{T}$ for the generation has not yet expired, do not transmit and wait for additional innovative packets. 


\section{Example: Conflicting Packets (Free Slot Available)}

In order to explain how the conflicting packets are resolved, we consider the following example. We assume that the generation size is 4 . Each packet is divided into equal sized symbols of 8 bits each. Suppose that a locally saved generation with generation ID 73098 has the decoding matrix given in Figure 4.7.

\begin{tabular}{|c|c|c|c|c|}
\hline Gen ID 73098 & Slot 0 & Slot 1 & Slot 2 & Slot 3 \\
\hline Source address & 1 & 2 & 3 & 0 \\
\hline Sequence Number & 1 & 3 & 6 & 0 \\
\hline
\end{tabular}

\begin{tabular}{|c|c|c|c|}
\hline 36 & 42 & 50 & 0 \\
\hline 48 & 62 & 64 & 0 \\
\hline 109 & 154 & 80 & 0 \\
\hline 0 & 0 & 0 & 0 \\
\hline
\end{tabular}

Figure 4.7: Generation with Symbol Location and Respective Coding Coefficients

The generation has 3 vectors stored locally. $(1,1),(2,3)$ and $(3,6)$ represent the 2 tuple (source address, sequence number) for each saved packet with their coefficients at slot 0,1 and 2 respectively. Slot 3 is empty.

We assume that the node with the above generation matrix received a packet for that generation with the coefficients and address / sequence number pair as given in Figure 4.8. 


\begin{tabular}{|c|c|c|c|c|}
\hline Gen ID 73098 & Slot 0 & Slot 1 & Slot 2 & Slot 3 \\
\hline Source address & 1 & 2 & 3 & 2 \\
\hline Sequence Number & 1 & 4 & 6 & 3 \\
\hline & 66 & 184 & 22 & 170 \\
\hline
\end{tabular}

Figure 4.8: Received Packet with Symbol Location and Respective Coefficients

The received packet has the 2 -tuple $(2,4)$ at slot 1 . However the locally saved generation has the coefficients for packet with 2-tuple $(2,3)$ at slot 1 . This conflict is resolved by moving the conflicting coefficients to slot 3 of the generation, which is free. Similarly, the 2-tuple $(2,3)$ in the received packet in slot 4 is moved to slot 1 of the locally saved generation. In this way, each node maintains a conflict-free generation.

\begin{tabular}{|c|c|c|c|c|}
\hline Gen ID 73098 & Slot 0 & Slot 1 & Slot 2 & Slot 3 \\
\hline Source address & 1 & 2 & 3 & 4 \\
\hline Sequence Number & 1 & 3 & 6 & 2 \\
\hline
\end{tabular}

\begin{tabular}{|c|c|c|c|}
\hline 36 & 42 & 50 & 0 \\
\hline 48 & 62 & 64 & 0 \\
\hline 109 & 154 & 80 & 0 \\
\hline 66 & 170 & 22 & 184 \\
\hline
\end{tabular}

Figure 4.9: Generation 73098 after Conflict Resolution

It should also be noted that due to this shuffle of coefficients, each node maintains the order of coefficients independent of other nodes and this order has local meaning only. Similarly, once the coefficients are moved to a particular slot, the respective coded 
symbols for that packet are also moved accordingly. After resolving the conflict, the new generation entries are given in Figure 4.9.

\section{Example: Conflicting Symbols (No Free Slot Available)}

In order to explain how the conflicting packets are resolved in case where no free slot is available in the generation, we consider the following example. We assume that the generation size is 4 . Suppose that the locally saved generation with generation ID 73098 has the decoding matrix given in Figure 4.10. The generation has 3 vectors stored locally. $(1,1),(2,3),(3,6)$ and $(4,2)$ represent the 2-tuple (source address, sequence number) for each saved packet with their coefficients at slot $0,1,2$, and 3 respectively.

\begin{tabular}{|c|c|c|c|c|}
\hline Gen ID 73098 & Slot 0 & Slot 1 & Slot 2 & Slot 3 \\
\hline Source address & 1 & 2 & 3 & 4 \\
\hline Sequence Number & 1 & 3 & 6 & 2 \\
\hline
\end{tabular}

\begin{tabular}{|c|c|c|c|}
\hline 36 & 42 & 50 & 69 \\
\hline 48 & 62 & 64 & 102 \\
\hline 109 & 154 & 80 & 09 \\
\hline 0 & 0 & 0 & 0 \\
\hline
\end{tabular}

Figure 4.10: Generation 73098 with Symbol Location and Respective Symbols

We assume that the node with the above generation matrix received a packet for that generation with the coefficients and address / sequence number pair as given in Figure 4.11. 


\begin{tabular}{|c|c|c|c|c|}
\hline Gen ID 73098 & Slot 0 & Slot 1 & Slot 2 & Slot 3 \\
\hline Source address & 1 & 2 & 3 & 4 \\
\hline Sequence Number & 1 & 3 & 7 & 2 \\
\hline
\end{tabular}

\begin{tabular}{|l|l|l|l|}
\hline 66 & 184 & 22 & 170 \\
\hline
\end{tabular}

Figure 4.11: Received Packet for Generation 73098

The received packet has the 2-tuple $(3,7)$ at slot 2 . However the locally saved generation has the coefficients for 2-tuple $(3,6)$ at slot 2 . This conflict is resolved by increasing the size of the generation and moving the conflicting packet's coefficients to the end. After resolving the conflict, the new generation entries are given in Figure 4.12.

\begin{tabular}{|c|c|c|c|c|c|}
\hline Gen ID 73098 & Slot 0 & Slot 1 & Slot 2 & Slot 3 & Slot 4 \\
\hline Source address & 1 & 2 & 3 & 4 & 3 \\
\hline Sequence Number & 1 & 3 & 6 & 2 & 7 \\
\hline
\end{tabular}

\begin{tabular}{|c|c|c|c|c|}
\hline 36 & 42 & 50 & 69 & 0 \\
\hline 48 & 62 & 64 & 102 & 0 \\
\hline 109 & 154 & 80 & 09 & 0 \\
\hline 66 & 184 & 0 & 170 & 22 \\
\hline
\end{tabular}

Figure 4.12: Generation 73098 after Conflict Resolution

\subsubsection{Decoding Process}

1. As we are dealing with broadcast messages, each node is required to decode the received encoded packets. 
2. Upon receiving an innovative packet, add the received packet to the last row of the decoding matrix.

3. If the rank is full, decode all the packets.

4. If the rank is not full, try to partially decode the matrix (i.e., check if the rank of a sub-matrix is full). If some packets are successfully decoded, send the decoded packets to the upper layer.

5. All 2-tuples for decoded packets are kept in a separate list for that generation. By doing so we make sure that the same packets are not sent to the upper layer again once the generation is partially or fully decoded again later.

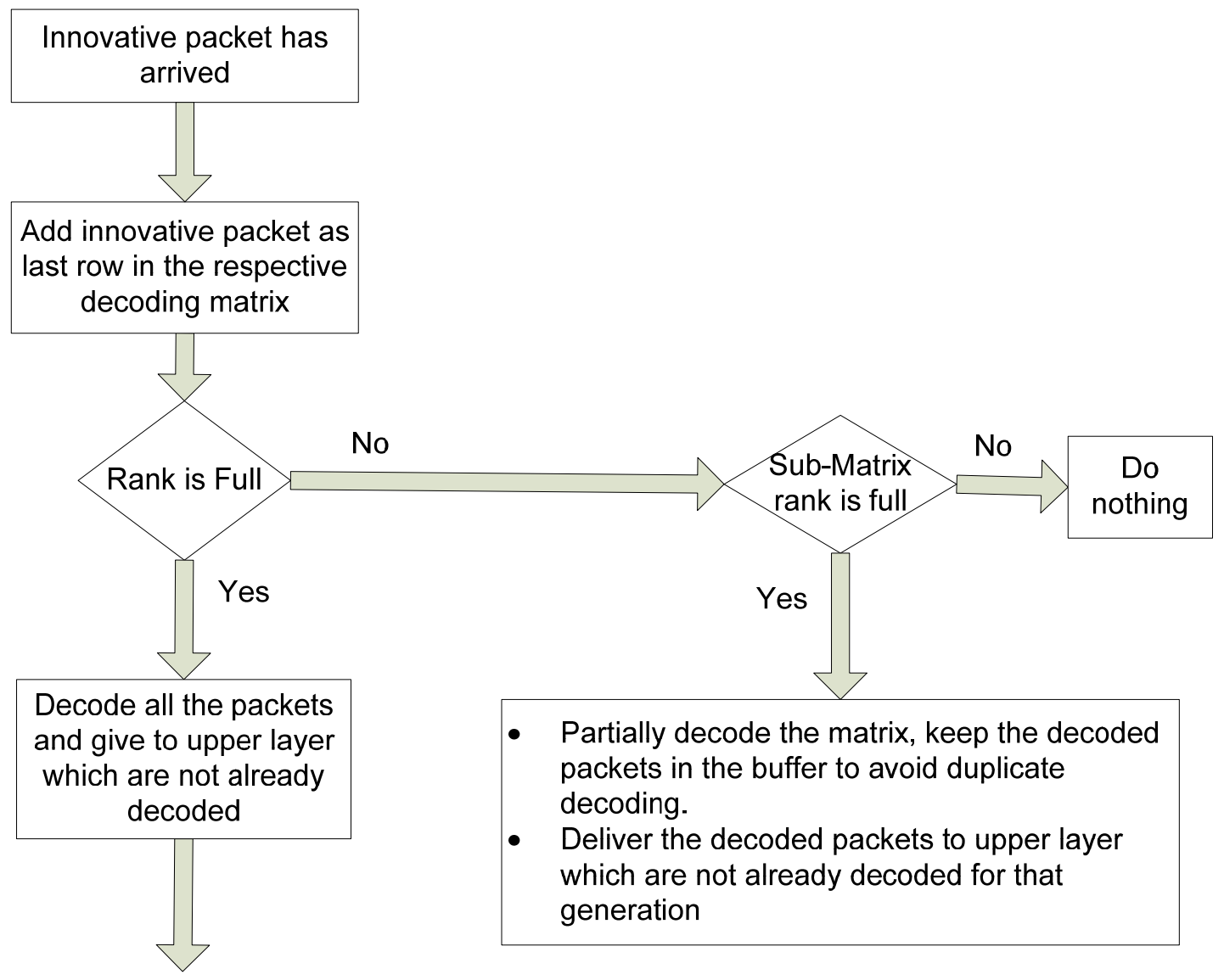

Figure 4.13: Flow Diagram - Decoding Process 


\section{- Early Decoding Example}

As mentioned before, we get a chance to decode the symbols without waiting for the generation to reach full rank. We get the chance to early decode the generations when a sub-matrix rank is full. Some of the examples illustrating the situations where a submatrix has full rank are given in Figure 4.14. The boxes indicate the sub-matrix with full rank.

$\left[\begin{array}{ccccc}48 & 112 & 212 & 231 & 30 \\ 23 & 44 & 108 \\ 34 & 54 & 0 & 0 & 0 \\ 23 & 0 & 0 & 0 & 0 \\ 3 & 0 & 0\end{array}\right],\left[\begin{array}{ccc}23 & 44 & 108 \\ 34 & 54 & 0 \\ 23 & 87 & 0\end{array}\right],\left[\begin{array}{ccccc}23 & 44 & 108 & 75 & 12 \\ \beta 4 & 54 & 212 & 0 & 0 \\ 23 & 105 & 98 & 0 & 0 \\ 90 & 45 & 20 & 0 & 0\end{array}\right]$

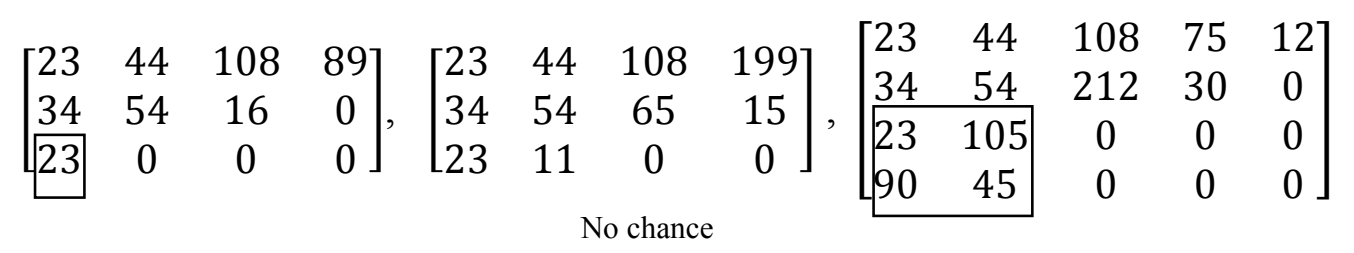

Figure 4.14: Early Decoding Examples

Early decoding has a major impact on reducing the end-to-end packet delay. Even if, by chance, the decoding matrix never reaches full rank due to the loss of packets, we can still decode some packets rather than losing all the packets of that generation. Similarly, if some packets have been received and we get the chance of early decoding, these packets do not have to wait for the generation to become full, which reduces the packet latency. However, early decoding can result in out-of-order decoding (and delivery to the higher layers) of packets. If the node is able to decode sequence number 4 
before sequence number 3 for some node, then the packets need to be reordered by the transport layer. 


\section{Chapter 5}

\section{Simulation Setup}

\subsection{Simulation Tool \& Parameters}

We have implemented our protocol in NS-2. The MAC protocol 802.11 is used and we have used the default parameters set in NS-2. The two-ray ground propagation model is used to simulate the propagation at the physical layer. The default values used in the simulations are provided in Table 5.1.

Mac set bandwidth_2Mb
Mac/802_11 set basicRate_1Mb
Mac/802_11 set dataRate_1Mb
Antenna/OmniAntenna set $\mathrm{X}_{-} 0$
Antenna/OmniAntenna set $Y_{-} 0$
Antenna/OmniAntenna set Z_ 1.5
Antenna/OmniAntenna set Gt_ 1.0
Antenna/OmniAntenna set Gr_ 1.0
Phy/WirelessPhy set CPThresh_10.0
Phy/WirelessPhy set CSThresh_1.559e-11
Phy/WirelessPhy set RXThresh_3.652e-10
Phy/WirelessPhy set bandwidth_2e6
Phy/WirelessPhy set Pt_0.28183815
Phy/WirelessPhy set freq_ $914 \mathrm{e}+6$
Phy/WirelessPhy set L_ 1.0

Table 5.1: Default values in NS2 
The default parameters specify that the total bandwidth is set to 2 Mbps where the rate for data frames is $1 \mathrm{Mbps}$ and rate for control frames is also $1 \mathrm{Mbps}$. Omnidirectional antenna is used by each mobile node and the antenna height is specified by Antenna/OmniAntenna set $Z_{-}$1.5. Transmit and receive antenna gain is set to 1 . CPThresh_, CSThresh_ and RXThresh_are important parameters and specify the collision threshold, carrier sense power and receive power threshold. The default values specify the maximum node reception range to be 250 meters. Since we are using Omnidirectional antenna, the node reception range forms a circle of 250 meter radius around the node. However the interference range / carrier sense range is $550 \mathrm{~m}$.

\subsection{Performance Metrics}

We ran each simulation for 500 second simulation time and averaged over 10 different runs. The performance metrics used to evaluate our algorithm are as following. PDR

Packet Delivery Ration (PDR) is the ratio of the total number of packets actually received by each node relative to the total number of packets that should be received ideally. Ideally, in case of broadcasting, all packets sent by each source should be received by all the nodes (including the sources) in the network. In case of network coding, only those packets are considered that are successfully decoded by each node. Merely receiving the packet does not mean that the received packet is meaningful, unless it is decoded successfully. The PDR is calculated as following

$P D R=($ Total packets received by each node $) /($ Total packets sent by each source $\times$ number of nodes) 


\section{End-to-end packet delay / latency}

End-to-end packet latency is the total time between sending a packet at the source and its successful reception at the receiver. In case of network coding it is the time between sending a coded packet at the source and its successful decoding at the receiver. For broadcasting, there are different ways to calculate the packet latency as all the nodes are receivers. We can calculate it in 3 different ways.

1) Maximum latency: For each packet transmitted, it is the maximum time it takes to receive that packet by any node. Ideally, nodes that are furthest from the source in terms of number of hops should have maximum latency. We calculate the maximum latency for each transmitted packet and then average it over the total number of packets sent by all the sources.

2) Minimum latency: For each packet transmitted, it is the minimum time it takes to receive that packet by any node provided that the node is not the source. If we include the source, minimum latency will always be 0 . We calculate the minimum latency for each transmitted packet and then average it over total number of packets sent by all the sources.

3) Overall average latency: To calculate the overall average latency, we first calculate the average of all the times for single packet sent and received by all the nodes in the network. The calculated average for each packet is again averaged over all the packets transmitted by all the sources.

In this thesis we used the overall average latency for comparisons. Average latency is a good measure of overall system latency and we deal with one parameter only rather than 2 parameters (Minimum \& Maximum) for latency. 


\section{MAC transmissions}

We further evaluate the performance of our protocol in terms of the total number of packet transmissions at the MAC layer. It includes both the data packets and control packets to justify the comparison with other protocols. Ideally, a given protocol will achieve high PDR and low latency with a low number of packet transmissions at the MAC layer, indicating low overheads and efficient use of the wireless media.

\subsection{Algorithms for Comparison}

We choose simple flooding, probabilistic flooding, BCAST and SMF for comparing the performance of our protocol using the above mentioned metrics.

1) For simple flooding, the packet is forwarded as soon as it reaches the node's network layer. Duplicate packets are discarded.

2) For probabilistic flooding, each node retransmits the received packets with probability $\mathrm{P}$. This significantly reduces the broadcast storm problem of simple flooding. It is very important that the right value of probability of retransmission is used that gives the optimal performance. For our scenarios, we found the following optimal values for different node densities and data rates. For each data point we need to find this optimal value as given in Table 5.2.

3) BCAST [30], which is based on the Neighbour Knowledge Method, exchanges periodic HELLO messages to collect 2-hop neighbourhood information. For retransmission, the receiving node $\mathrm{A}$ reschedules the packet with random delay if all the neighbours of A are not covered by the previous hop B of the received packet. If the same packet arrives from another neighbour (or set of neighbours) $\mathrm{C}$ 
who covers the remaining neighbours, A discards the packet. The delay is calculated by multiplying the uniformly distributed randomly generated number by the ratio of the highest number of neighbours of neighbours of A divided by total number of neighbours of A and the scaling factor for BCAST.

\begin{tabular}{|c|c|c|c|c|c|c|c|}
\hline \multicolumn{8}{|c|}{ Static Scenarios } \\
\hline \multicolumn{2}{|c|}{01 - Source } & \multicolumn{2}{|c|}{04 - Source } & \multicolumn{2}{|c|}{01 - Source } & \multicolumn{2}{|c|}{04 - Source } \\
\hline Rate & $P$ value & Rate & P Value & Nodes & P Value & Nodes & P Value \\
\hline 1 & 0.5 & 1 & 0.4 & 05 & 0.6 & 25 & 0.3 \\
\hline 50 & 0.25 & 25 & 0.20 & 25 & 0.5 & 50 & 0.15 \\
\hline 100 & 0.15 & 50 & 0.15 & 50 & 0.25 & 75 & 0.1 \\
\hline & & & & 75 & 0.2 & 100 & 0.1 \\
\hline & & & & 100 & 0.1 & & \\
\hline \multicolumn{8}{|c|}{ Mobile Scenarios } \\
\hline \multicolumn{2}{|c|}{01 - Source } & \multicolumn{2}{|c|}{04 - Source } & \multicolumn{2}{|c|}{01 - Source } & \multicolumn{2}{|c|}{04 - Source } \\
\hline Rate & $\mathrm{P}$ value & Rate & P Value & Nodes & P Value & Nodes & P Value \\
\hline 1 & 0.45 & 1 & 0.3 & 05 & 0.6 & 25 & 0.25 \\
\hline 50 & 0.20 & 25 & 0.1 & 25 & 0.5 & 50 & 0.15 \\
\hline 100 & 0.1 & 50 & 0.1 & 50 & 0.25 & 75 & 0.1 \\
\hline & & & & 75 & 0.2 & 100 & 0.1 \\
\hline & & & & 100 & 0.1 & & \\
\hline
\end{tabular}

Table 5.2: Optimal P values used for Probabilistic Flooding 
4) The Simplified Multicast Forwarding (SMF) algorithm [33] implements the Neighbour Knowledge distributed method of dynamically electing a reduced relay set of neighbours for broadcasting the information. The SMF architecture consists of three main components; neighborhood discovery, relay set selection and forwarding process with duplicate packet detection mechanism. Source based Multi Point Replay (S-MPR) is used as relay set selection algorithm.

\subsection{Sensitivity of ARLNCCF}

We investigated the behavior/sensitivity of our protocol to Generation Size, Generation Timeout, and its performance with/without early decoding. The results are explained and analyzed in the following section.

\subsubsection{Generation Size}

\section{Source Scenario}

1-Source, 50 nodes, date rate 50 kbps. Timeout value: 0.1, GD Threshold: 1

Figure 5.1 shows the PDR as a function of Generation Size for 01 source scenario. The PDR initially is at around $80 \%$ which slowly increases to approximately $97-99 \%$ as the generation size increases. For generation size of 6 and 8 , we observe the highest PDR. The reason for lower PDR for smaller generation sizes is that the generations are complete before the timeout occurs after 0.1 second. Once the generation is complete, the nodes re-encode the generations and transmit $\mathrm{N}_{\mathrm{T}}$ packets. Since the generation size is very small, the $\mathrm{N}_{\mathrm{T}}$ value, which is dependent on generation size, is also very small. If the nodes have a dense neighbourhood, $\mathrm{N}_{\mathrm{T}}$ becomes the probability with which the nodes 
transmit. Nodes re-encode and transmit the encoded packets with very small probability of retransmission. As a result, some of the nodes never get a chance to completely decode the generation. Similarly, after the re-encoding operation, with smaller generation sizes, the chances of receiving more innovative packets is also very much reduced. As the generation size further increases to 6 and above the PDR improves. The latency is more or less constant as the generation size increases as shown in Figure 5.2. The reason for this is that we are doing early decoding and most of the packets get the chance to be decoded early.

In this research work, since we are more focused on multi-source scenarios, we have done more investigation of the sensitivity of the protocol for multi-source scenarios.

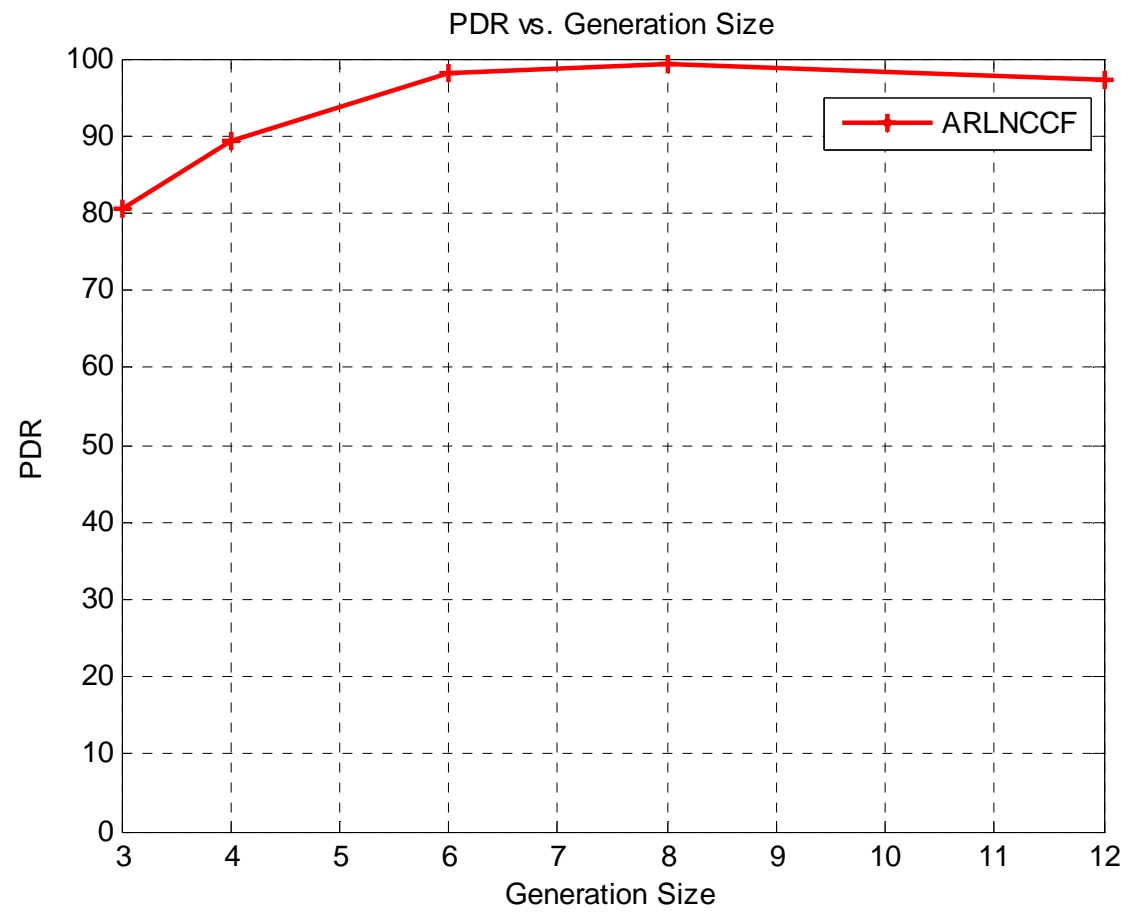

Figure 5.1: PDR vs. Generation Size (01 Source) 


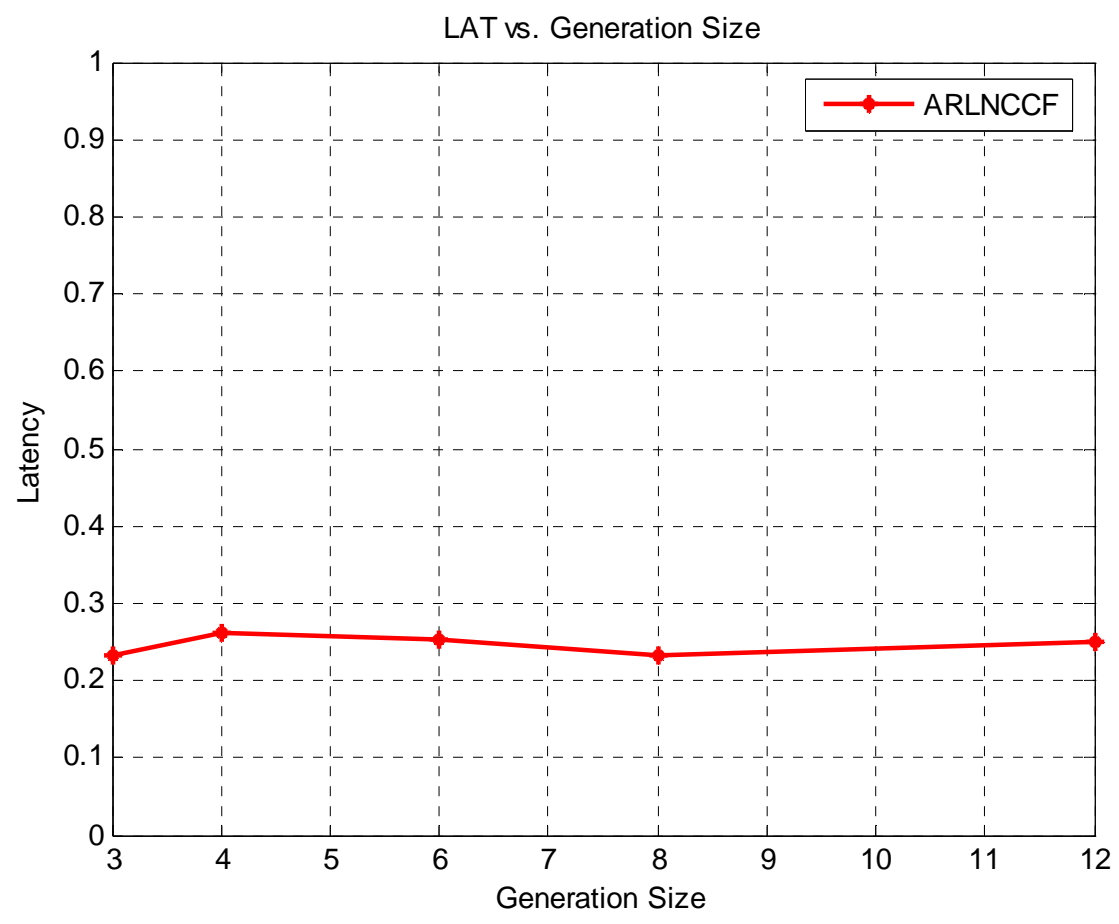

Figure 5.2: Latency vs. Generation Size (01 Source)

\section{Source Scenarios}

4-Source, 50 nodes, date rate 25kbps per source. Timeout value: 0.1, GD Threshold: 1

Figure 5.3 shows PDR as a function of Generation Size. It is observed that the PDR is around $80 \%$ for a very small generation size and it increases to around $96 \%$ for a generation size 4. PDR starts to decline again as the generation size increases. For a timeout value of 0.1 seconds, a generation size of 4 gives the best performance in terms of PDR. 


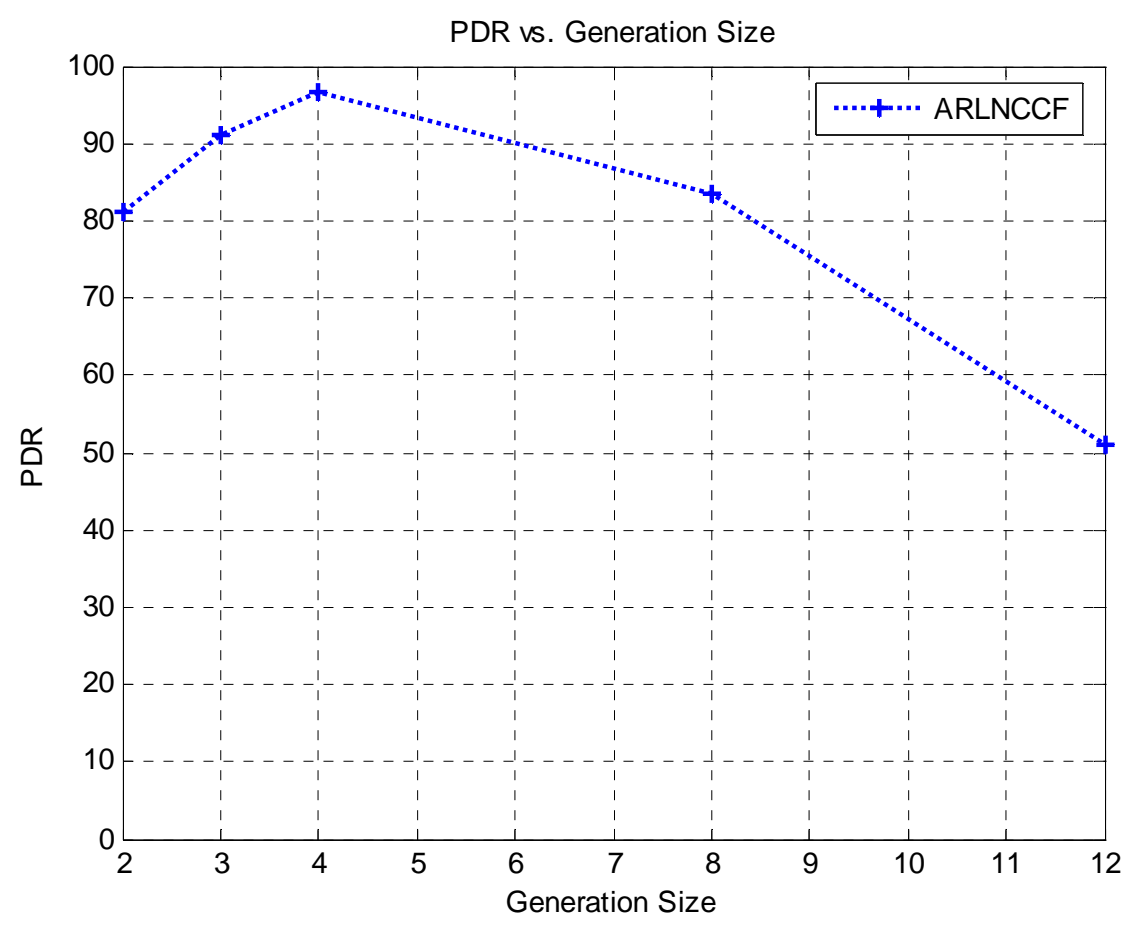

Figure 5.3: PDR vs. Generation Size (04 Sources)

For very small generation sizes, we are not getting any real benefit of network coding. We calculate the required number of retransmissions for the re-encoded packets, $N_{T}(i)=$ Igeneration size $/ \operatorname{Min}\left(N r_{N n}(m)\right.$, for all $\left.n\right) 7$, so a very small generation sizes causes the ratio to result in a very small probability of retransmission (which adds to the lower PDR value). Secondly, since the generation size is very small, the probability that the nodes will receive more innovative packets later on (after $\mathrm{N}_{\mathrm{T}}$ transmissions) is also reduced. So nodes do one re-encoding operation after the generation is full (transmit with probability $\mathrm{N}_{\mathrm{T}}$ ) and afterwards refrain from re-encoding as they do not receive any further innovative packets even if their neighbours require one more packet to decode their generation. This results in lower PDR and also high latency as some generations 
wait longer to receive the innovative packets from any neighbouring node to decode the generation fully. Figure 5.4 shows latency as a function of Generation Size.

Mathematically, if a node received $a x+b y=c$, it has to wait for another innovative packet to solve the equation. For very small generation sizes, this probability (to receive more innovative packets) is reduced. Even with early decoding, we cannot decode the packets from a single equation with 2 unknowns and need to wait for another innovative packet. Overall, a very small generation size causes fewer MAC transmissions but causes higher latency and lower PDR.

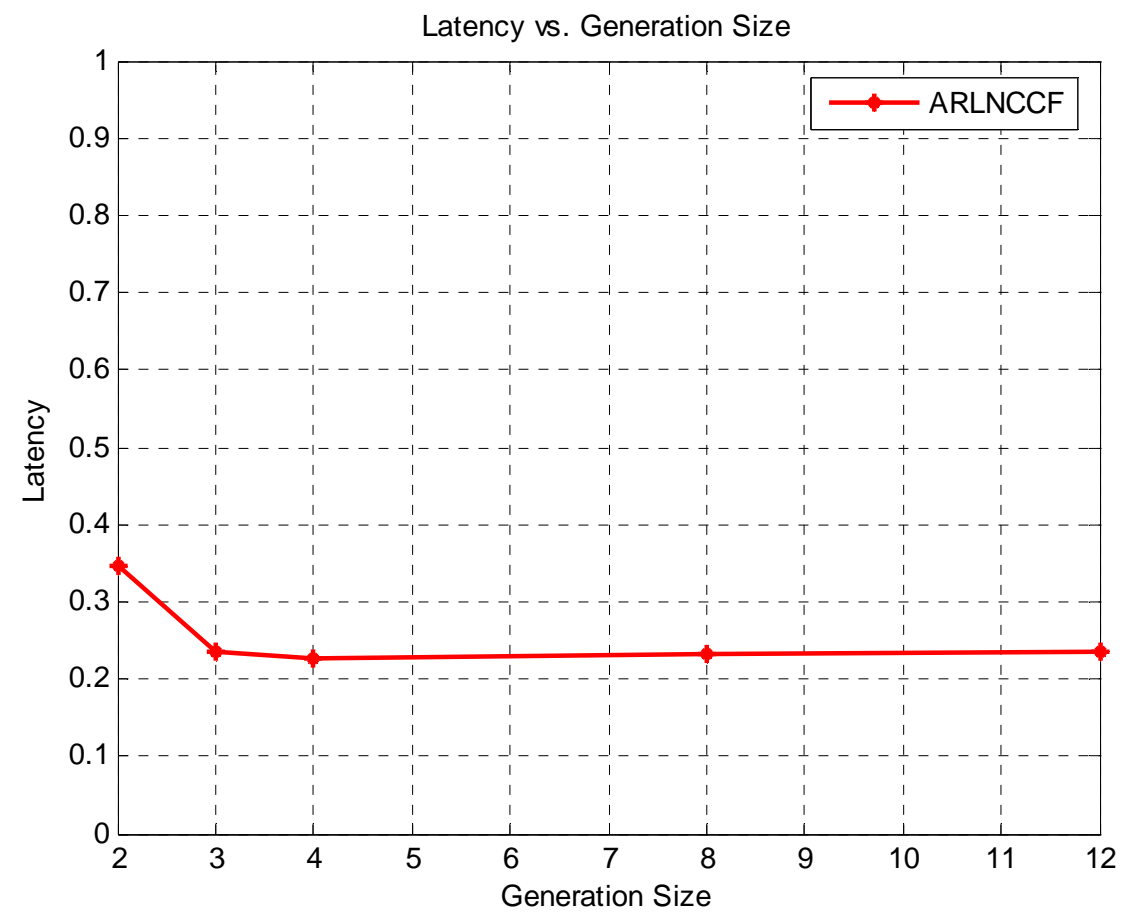

Figure 5.4: Latency vs. Generation Size (04 Sources)

Figure 5.4 shows that as the generation size increases, the latency is more or less constant. The reason for this is that we are supporting early decoding and there is always a chance to decode the coded packets early. There are many packets that do not get 
decoded when the generation size is big. Consequently the PDR is very low for larger generation sizes. The reason for this behavior is that for bigger generation sizes, at the generation timeout which set to 0.1 second, the nodes transmit $\mathrm{N}_{\mathrm{T}}$ coded packets to the neighbours. In fact the coded packets do not have complete generation information as the generation is not complete yet. Due to the phenomenon of early decoding, we are able to decode a few of the packets.

Subsequently, when the node receives more innovative packets, and if by chance any innovative packet is missed, the node is not able to decode the remaining packets. Even the early decoding does not help in that case. This can be explained with the following example. Suppose we have generation size 8 . At the timeout, we assume that the generation is half complete. $\mathrm{N}_{\mathrm{T}}$ packets are transmitted, which combine information from a few packets (less than generation size). With early decoding, we are able to decode these packets, $a, b, c \& d$. The coefficients of these packets are shown in (1) as the last four rows of the decoding matrix. Once more innovative packets arrive and if any transmission is missed or results in non-innovative packet, the chances to decode the remaining packets are minimized. In the following example, the node received 3 more coded packets for the same generation and stored their coefficients in the generation matrix (1) as the first 3 rows of the decoding matrix. We are not able to decode $e, f, g \& h$ even with early decoding as the generation is missing one innovative packet. As a result the PDR goes down. The more the generation size increases, the higher the chances of being left with few coded packets (which we are unable to decode). 


$$
\left[\begin{array}{cccccccc}
h 7 & g 7 & f 7 & e 7 & d 7 & c 7 & b 7 & a 7 \\
h 6 & g 6 & f 6 & e 6 & d 6 & c 6 & b 6 & a 6 \\
0 & g 5 & f 5 & e 5 & d 5 & c 5 & b 5 & a 5 \\
0 & 0 & 0 & 0 & d 4 & c 4 & b 4 & a 4 \\
0 & 0 & 0 & 0 & d 3 & c 3 & b 3 & a 3 \\
0 & 0 & 0 & 0 & 0 & c 2 & b 2 & a 2 \\
0 & 0 & 0 & 0 & 0 & 0 & b 1 & a 1
\end{array}\right]
$$

As far as MAC transmissions are concerned, as shown in Figure 5.5, we observe that as the generation size increases, there are more and more MAC transmissions. The reason for this behavior is that at generation timeout, which is set to 0.1 seconds, the node transmits $\mathrm{N}_{T}$ copies to its neighbors. $\mathrm{N}_{\mathrm{T}}$ is dependent on the generation size. As we increase the generation size, the $\mathrm{N}_{\mathrm{T}}$ value increases.

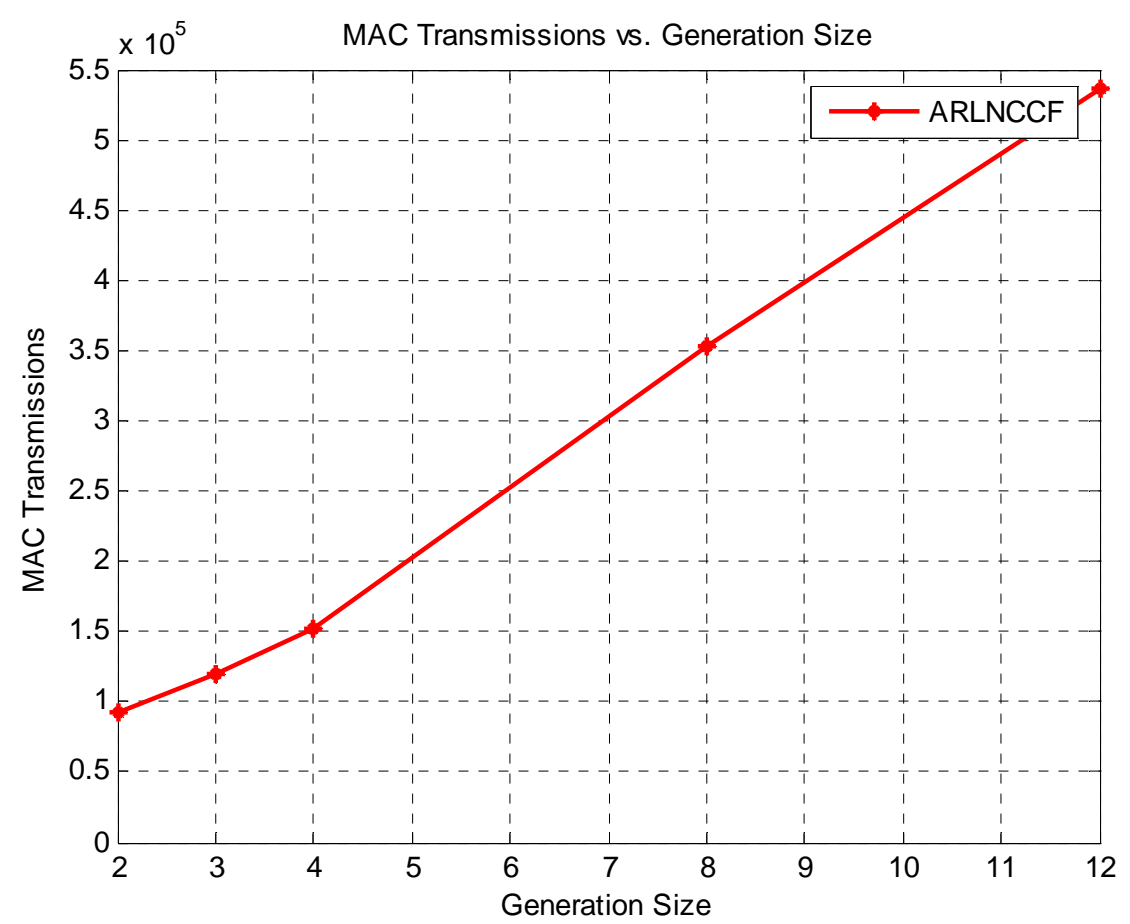

Figure 5.5: MAC Transmissions vs. Generation Size (04 Sources) 
In our protocol development we assume that at generation timeout, the generation is full or close to full. At generation timeout, assuming that a generation is almost complete, the node transmits $\mathrm{N}_{\mathrm{T}}$ copies to its neighbors. However, for larger generation sizes, the generation is not yet complete at 0.1 second. This results in many redundant transmissions which contribute very little in completely decoding the generation. Similarly, there are many innovative packets received even after the expiration of the timeout value for larger generation sizes. They need to be rebroadcasted also after $\mathrm{N}_{\mathrm{T}}$ transmissions, which results in a higher number of MAC transmissions.

In a nutshell, there is a need to maintain a balance between the timeout value as well as the generation size. Larger generation sizes also cause significant overhead as the coded packet header needs to carry the source address and sequence number of each original packet.

\subsubsection{Generation Timeout}

\section{Scenario}

\section{4-Source, 50 nodes, date rate 25kbps per source, Generation size: 4}

Figure 5.6 shows PDR as a function of Generation Timeout. It is observed that the PDR is around $90 \%$ for very small timeout values. It increases to around $96 \%$ for a timeout value of 0.1 second and then starts to decline again to $90 \%$. The reason for this behavior is that for very low generation timeout values, the nodes re-encode the generations at timeout, transmit $\mathrm{N}_{\mathrm{T}}$ copies even if the generation is not complete and still require a few more coded packets to complete the generation. Later on additional innovative packets arrive; the node just transmits one re-encoded packet. 


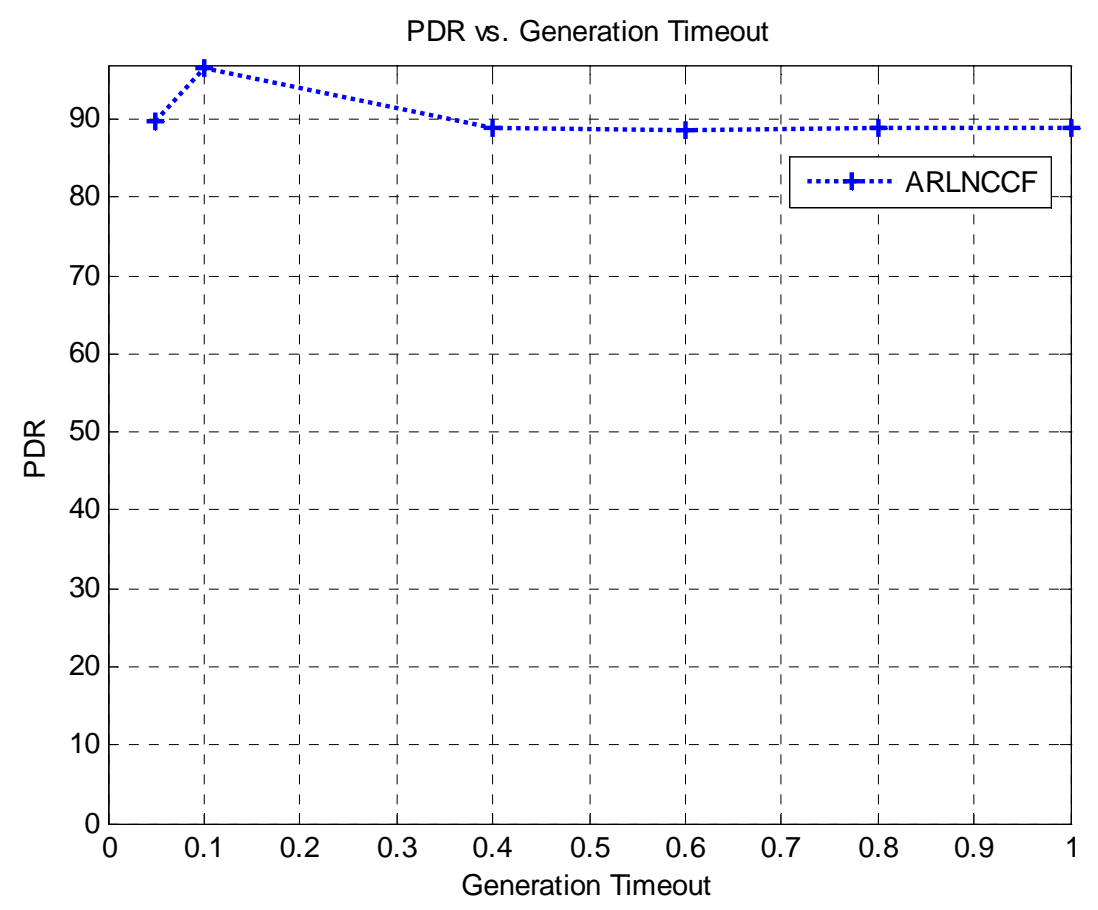

Figure 5.6: PDR vs. Generation Timeout

$\mathrm{N}_{\mathrm{T}}$ packet transmissions make sense once the generation is almost complete and a node is able to decode almost all packets in a generation. However, for very low values of the generation timeout, the neighbouring nodes just receive $\mathrm{N}_{\mathrm{T}}$ packets which do not have enough information for all the packets in that generation and nodes have to wait for more innovative packets. The situation is almost similar to the case discussed above where the generation size is large and at timeout the generation is not complete. Here the generation size is 4 but the timeout value is so small that the generation is not complete at timeout. As such, after $\mathrm{N}_{\mathrm{T}}$ transmissions, the nodes keep on waiting for more innovative packets to fully decode the generation. If any innovative packet is missed or dropped, we will be left with few packets that we are not able to decode even with early decoding. Similarly, the received innovative packet for some nodes does not guarantee that the same packet is 
innovative for other nodes as well. If some generations receive all innovative packets by chance, nodes are able to decode the generation fully. However some of the packets are decoded with a delay after receiving innovative packets later on. This results in higher latency as shown in Figure 5.7. At a timeout value of 0.1 second, the PDR improves and latency is reduced. It is however observed that we have a higher number of MAC transmissions in order to achieve higher PDR and lower latency.

There needs to be a balance between generation size as well as timeout value. Once we further increase the generation timeout value, the PDR declines to around $90 \%$ and then remains constant. Similarly, the latency starts to increase slightly as the timeout value increases. The reason for this behavior is that the timeout value is set such that at this value, even if the generation is not complete due to either delays or drop of packets, the nodes need to re-encode and transmit $\mathrm{N}_{\mathrm{T}}$ packets. For higher timeout values, if the generation is not complete by any chance before the timeout value, the nodes keep on waiting for the timeout to expire before they can re-encode and transmits the packets for that generation. This causes higher latency.

Figure 5.8 shows MAC transmissions as a function of Generation Timeout. For higher timeout values, the nodes retransmit only when the generation is complete. The reason for this is that most of the generations get completed before the timeout expires. The generation is complete once it has received all innovative packets and the number of rows equals the number of columns in the decoding matrix. This causes fewer MAC transmissions but results in slightly lower PDR and slightly higher latency. In a nutshell, these parameters strike a compromise between one performance metric and another. 


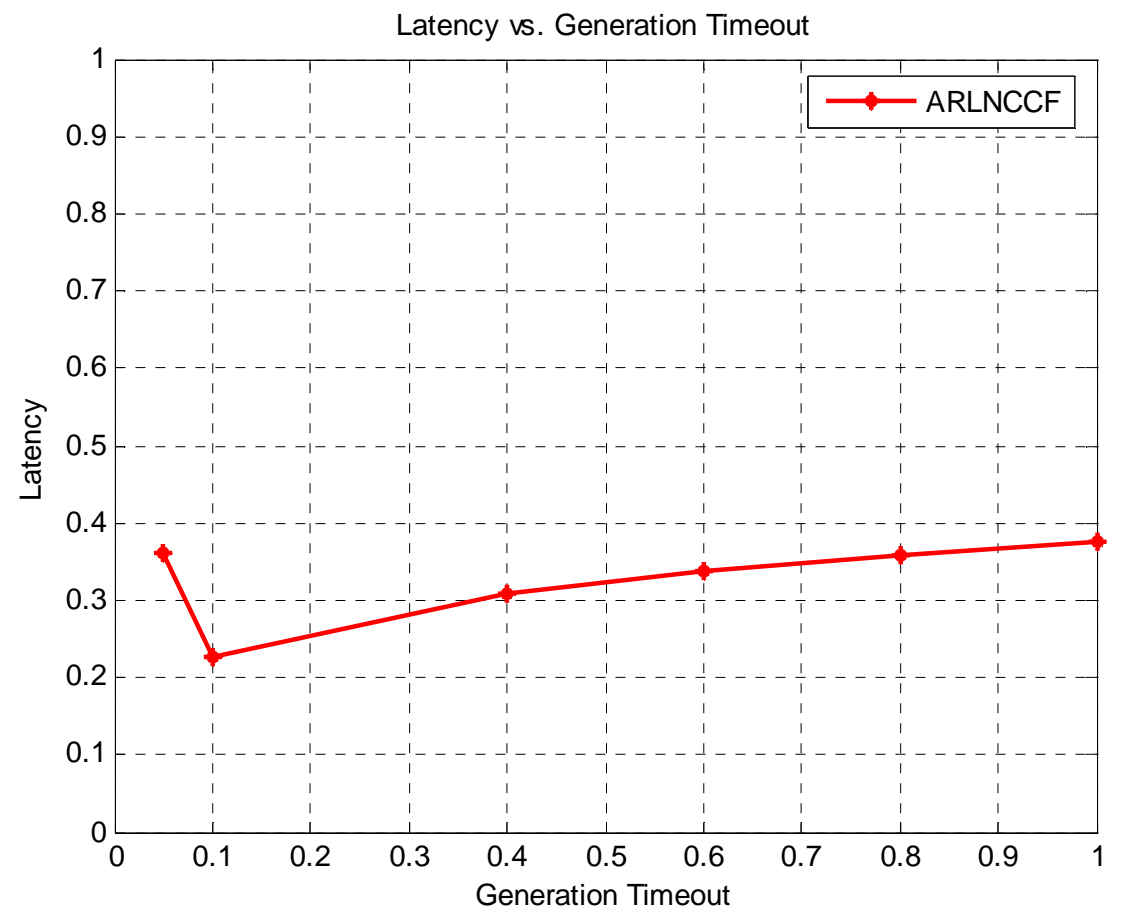

Figure 5.7: Latency vs. Generation Timeout

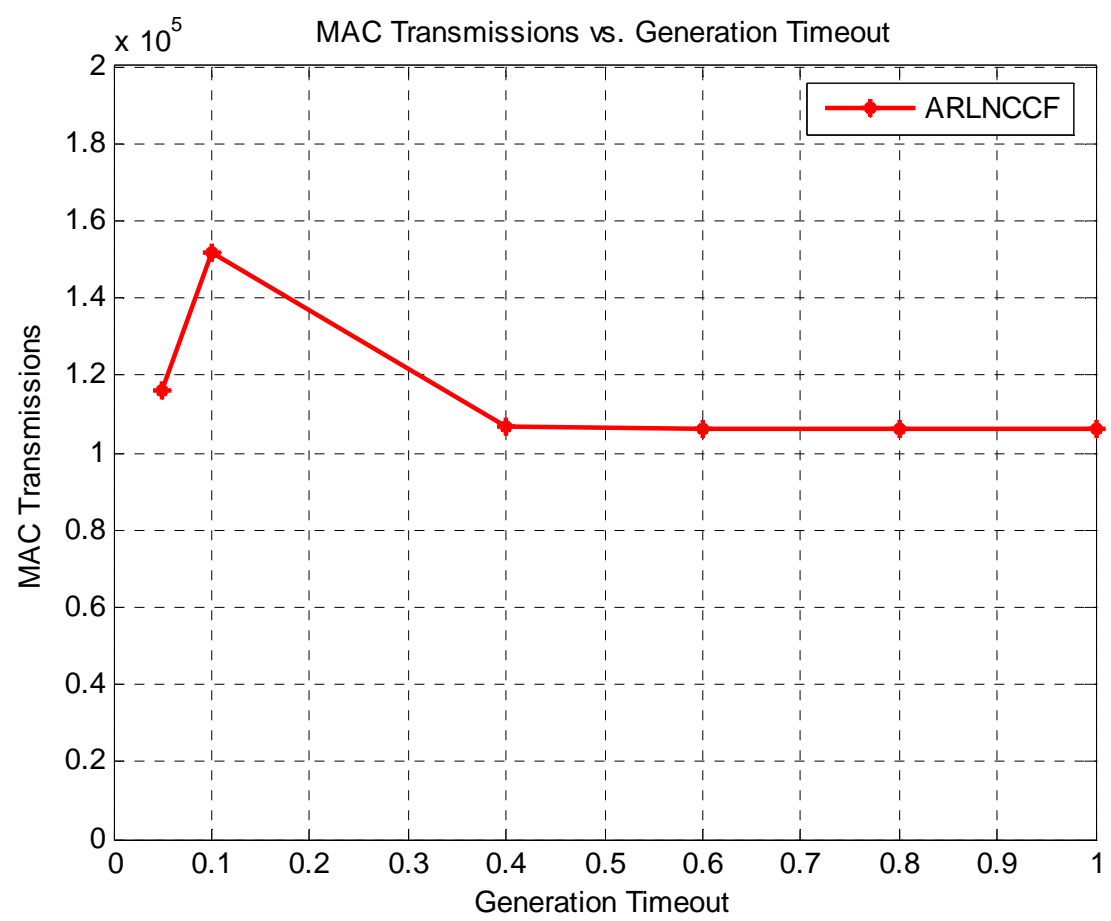

Figure 5.8: MAC Transmissions vs. Generation Timeout 


\subsubsection{Early Decoding}

\section{1-Source Scenarios - Varying data rate}

\section{Scenario}

(01-Source (Static), Nodes 50-Generation size 6, GD Threshold 2, Timeout 0.1)

As shown in Figure 5.9, there is almost no difference in PDR with and without early decoding. The major impact is on the latency as shown in Figure 5.10. At very low data rates, we see that it takes much longer time to complete the generations and as a result the latency is quite high for cases that do not support early decoding. For very low data rates, at a generation timeout of 0.1 second, very few packets are generated (fewer than the generation size). After $\mathrm{N}_{\mathrm{T}}$ transmissions, the neighbouring nodes need to wait for more innovative packets to fully decode the generation (without early decoding).

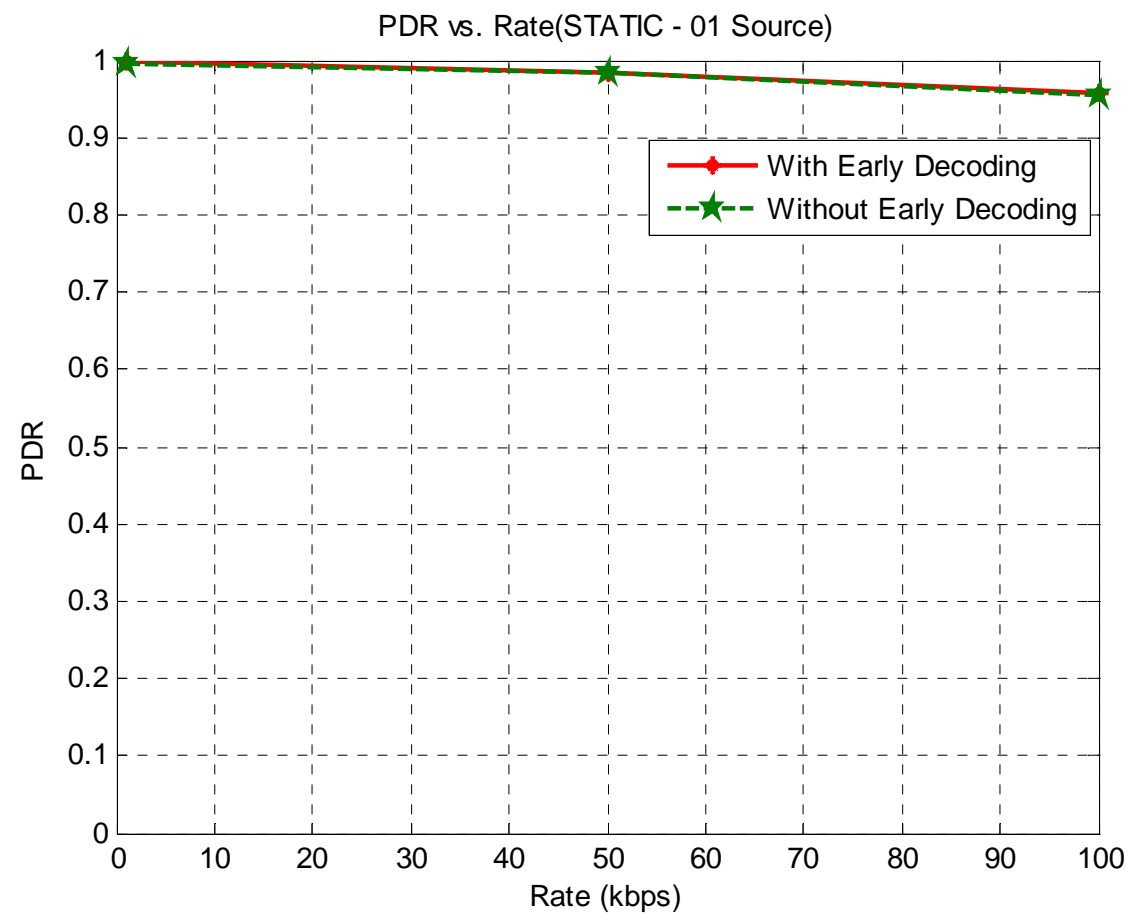

Figure 5.9: PDR vs. Rate (kbps) - (Static - 01 Source) 
With early decoding, we always get the chance to decode the sub-matrix. However in the absence of early decoding, the nodes receive very few packets after the timeout for very low data rates and keep on waiting for more innovative packets. Since the data rate is very low, the innovative packets also arrive at a very slow rate. Once all the innovative packets arrive and a generation is complete, the node decodes the generation successfully.

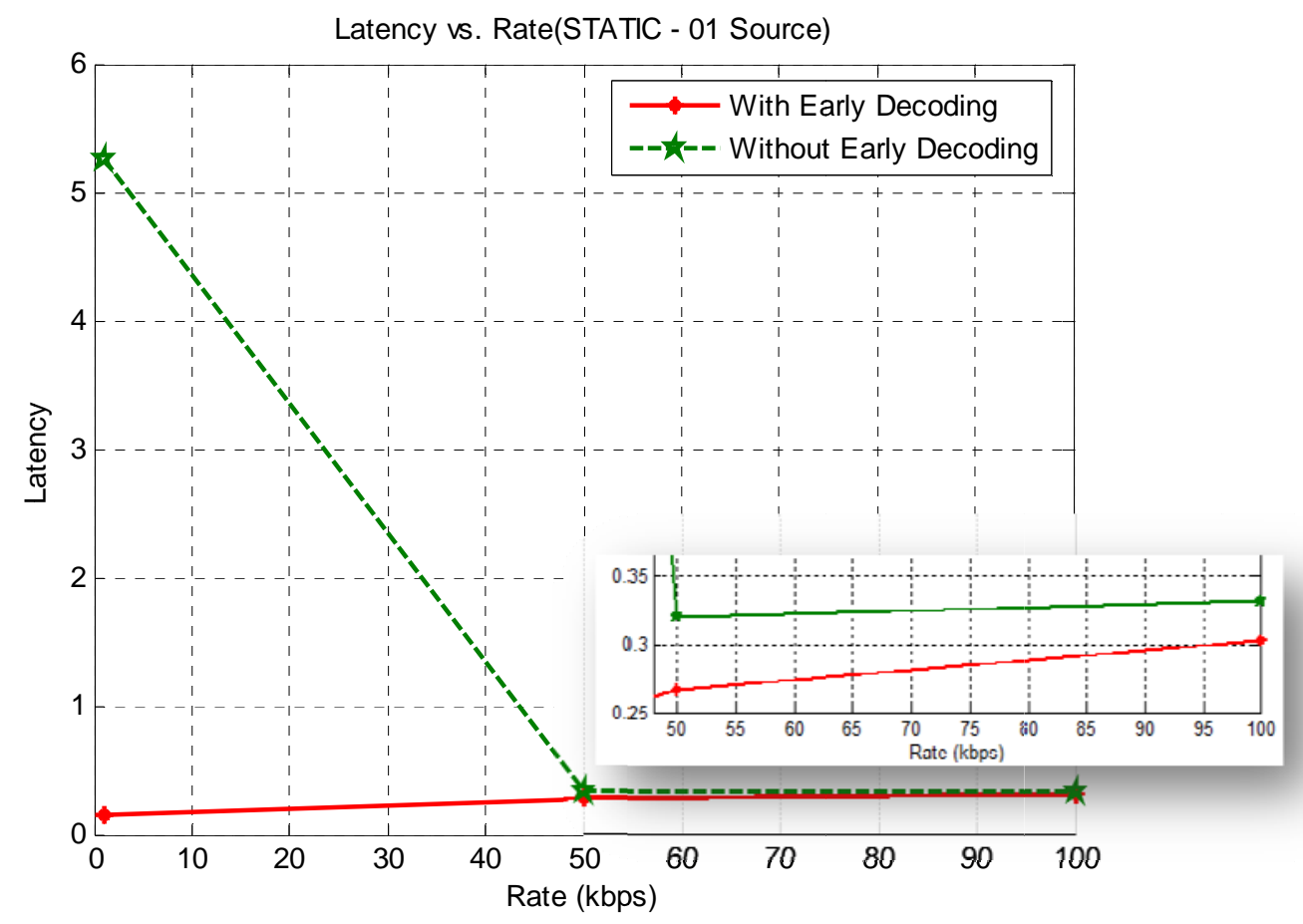

Figure 5.10: Latency vs. Rate (kbps) - (Static - 01 Source)

Overall, this leads to a PDR which is almost the same as with early decoding but results in very large decoding delays. As the data rate increases to beyond $50 \mathrm{kbps}$, higher data rates cause the generations to also complete quickly and the difference between with and without early decoding becomes less significant. The difference still exists but reduces to $0.05-0.15$ second as shown in Figure 5.10. 


\section{1-Source Scenarios - Varying Number of Nodes}

Scenario

(01-Source (Static), Rate 50 - Generation size 6, GD Threshold 2, Timeout 0.1 second)

When we investigate the performance by varying the number of nodes, PDR is almost the same for both cases as shown in Figure 5.11.

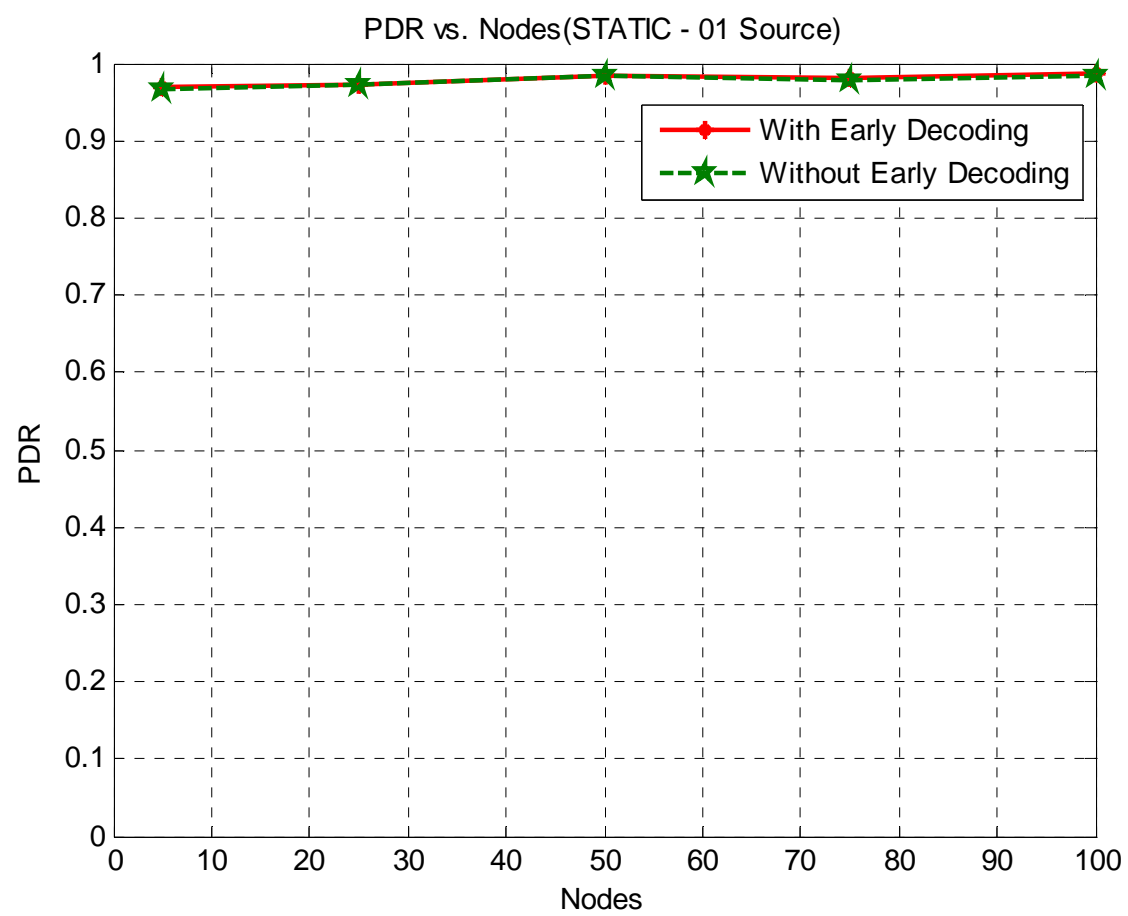

Figure 5.11: PDR vs. Nodes (Static - 01 Source)

However, the major impact is seen in the latency of the packets as shown in

Figure 5.12. There is an additional delay of $0.1-0.15$ second between with and without early decoding. With early decoding, the packets are consistently received much earlier than without early decoding case. The reason is already explained above when we discussed the cases with varying rate. It is noticed that varying the node densities has no impact on with and without early decoding cases. The difference in latency here is observed due to our set data rate of $50 \mathrm{kbps}$ and not due to varying the node densities. 


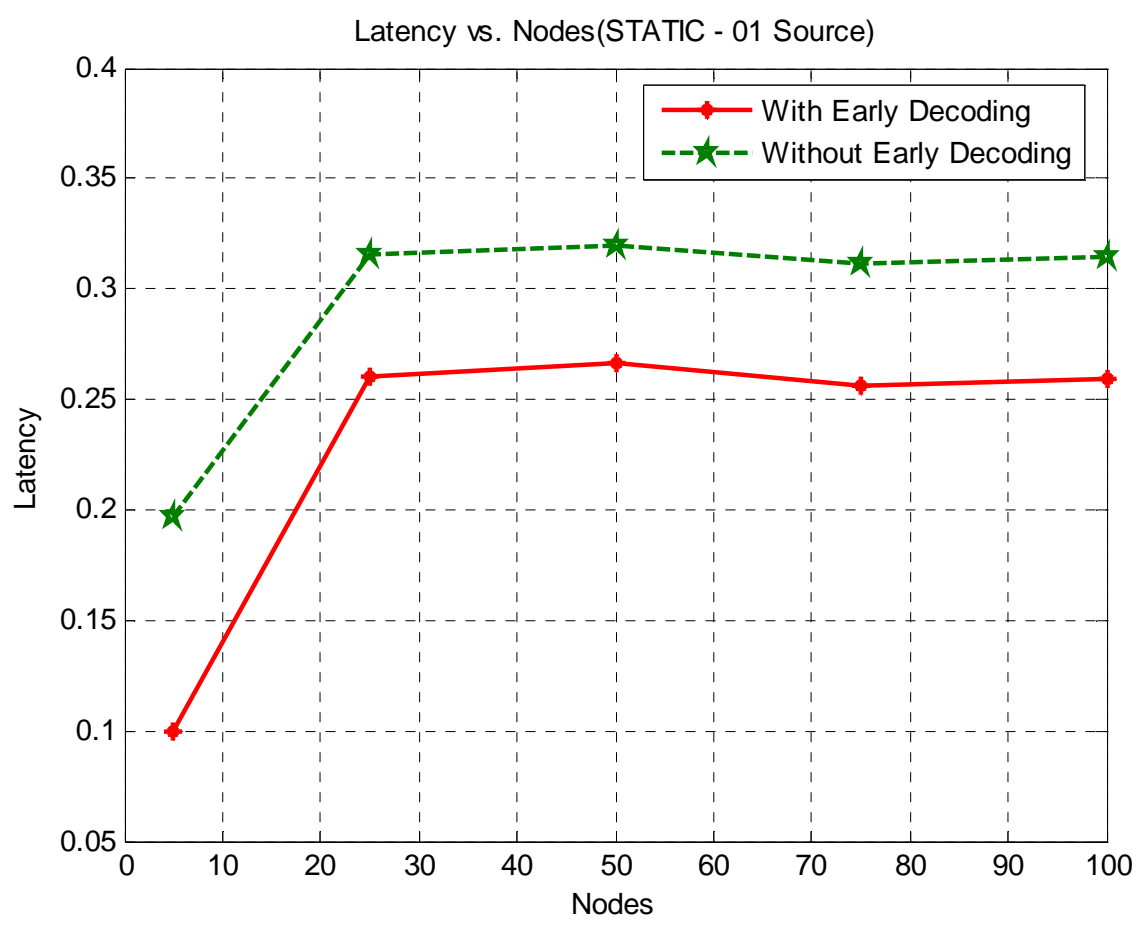

Figure 5.12: Latency vs. Nodes (Static - 01 Source)

\section{4-Source Scenarios - Varying Rate}

\section{Scenario}

\section{(04-Source (Static), Nodes 50 - Generation size 4, GD Threshold 1, Timeout 0.1)}

The results for scenarios with 4 sources are almost similar to those of single source scenarios with the difference that after $25 \mathrm{kbps}$ rate, the latency is almost the same for both cases of with and without early decoding as shown in Figure 5.14. Before the 25 kbps data rate, the case without early decoding has much higher latency due to low data rates. It takes more time to complete the generation fully and then decode. Early decoding shows a steady performance and shows no impact due to different data rates. The reason for this behavior is already explained when discussing the single source scenarios. 


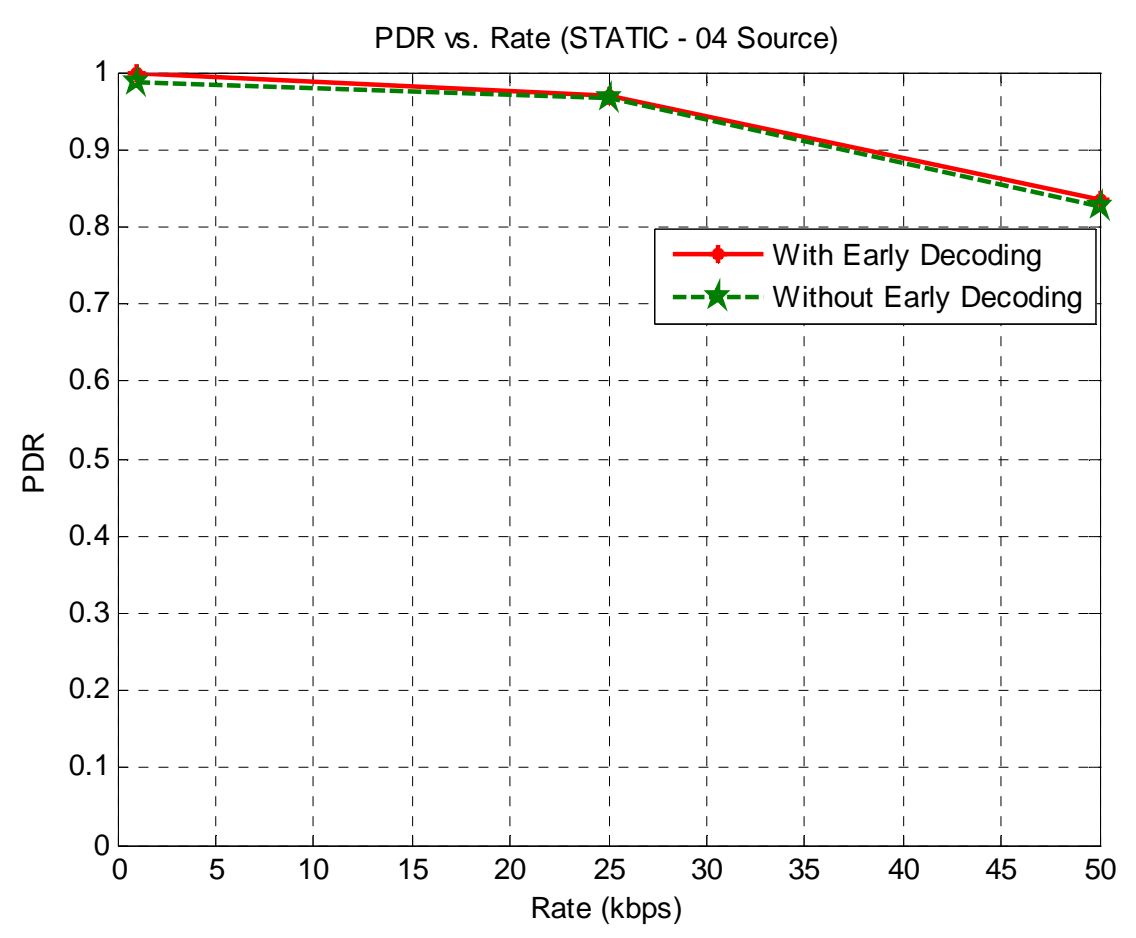

Figure 5.13: PDR vs. Rate (kbps) - (Static - 04 Source)

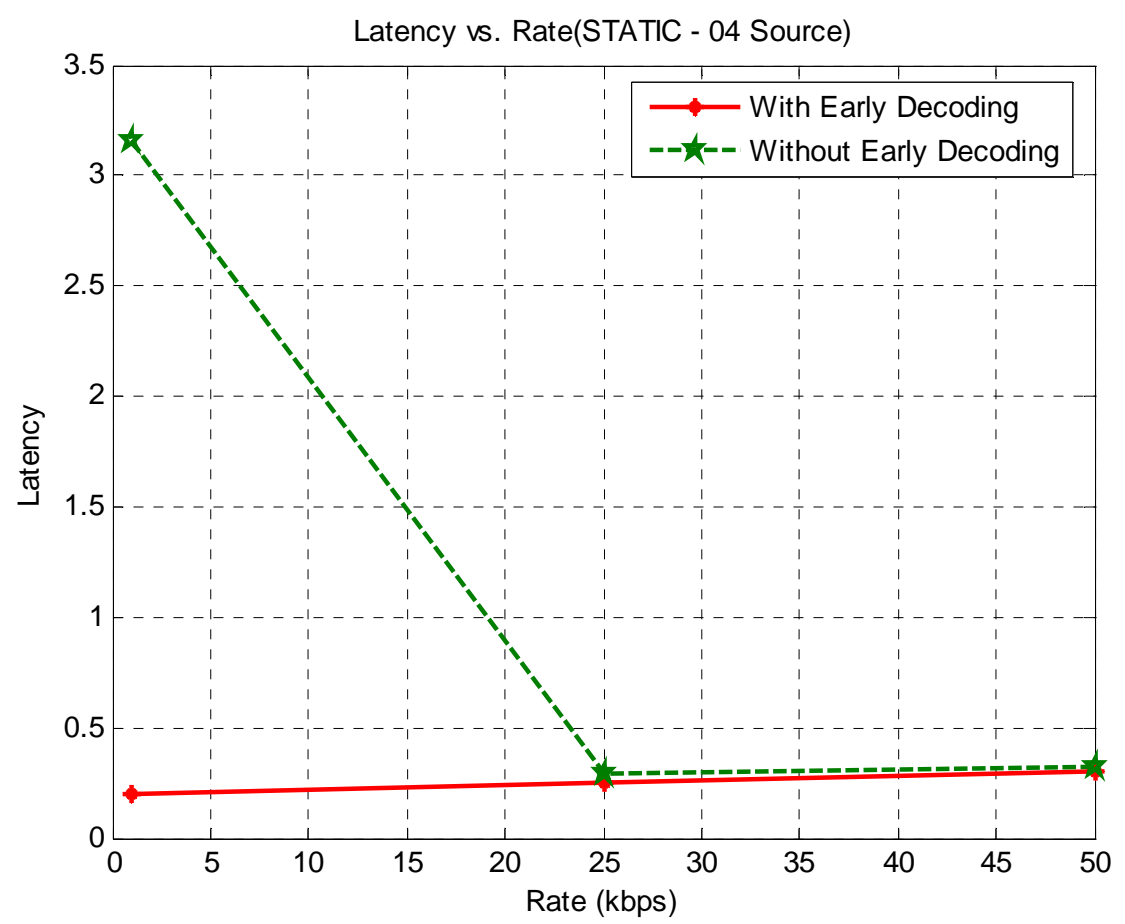

Figure 5.14: Latency vs. Rate (kbps) - (Static - 04 Source) 


\section{4-Sources Scenarios - Varying Number of Nodes}

\section{Scenario}

(04-Source (Static), Rate 25 - Generation size 4, GD Threshold 1, Timeout 0.1 second)

Keeping the data rate at $25 \mathrm{kbps}$ and varying the number of nodes, we observe that there is a consistent additional delay between with and without early decoding cases as shown in Figure 5.16. We are always able to decode the packets early and achieve lower latency for different node densities.

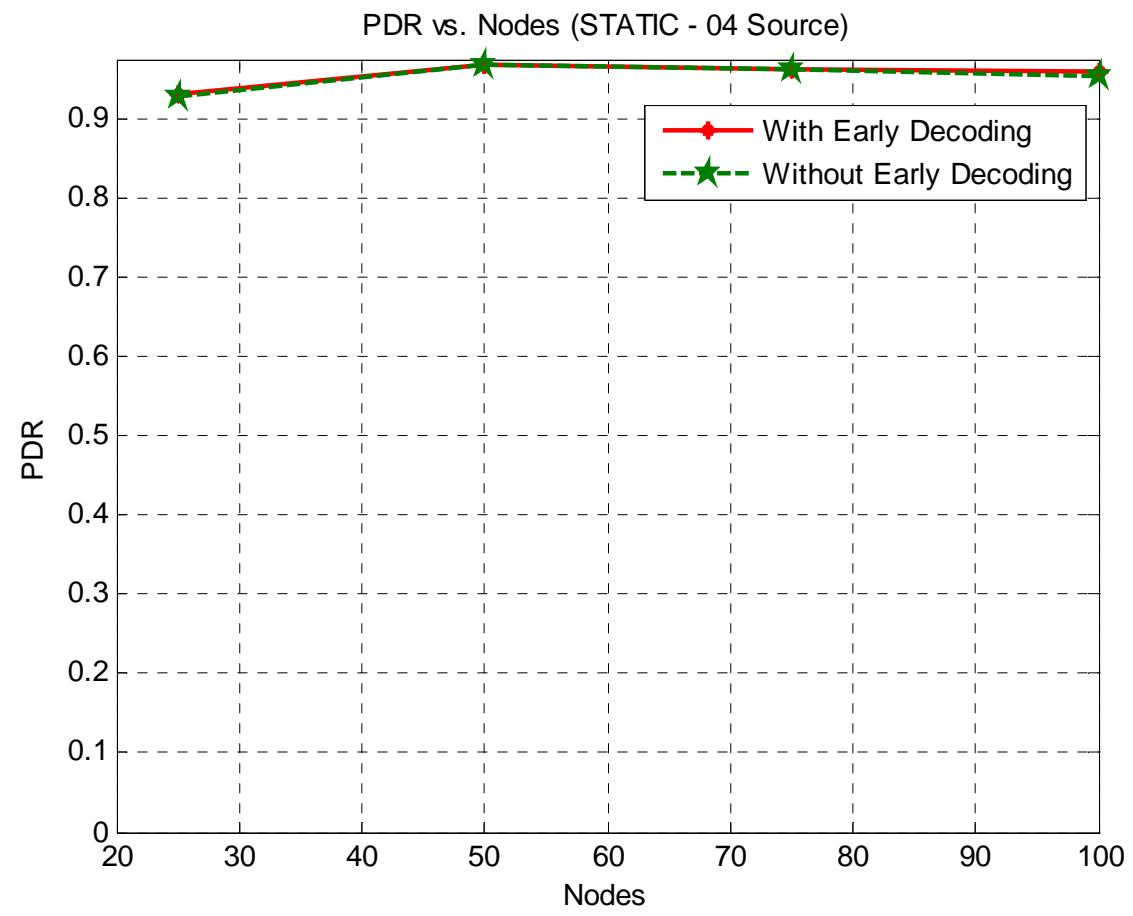

Figure 5.15: PDR vs. Nodes (Static - 04 Sources) 


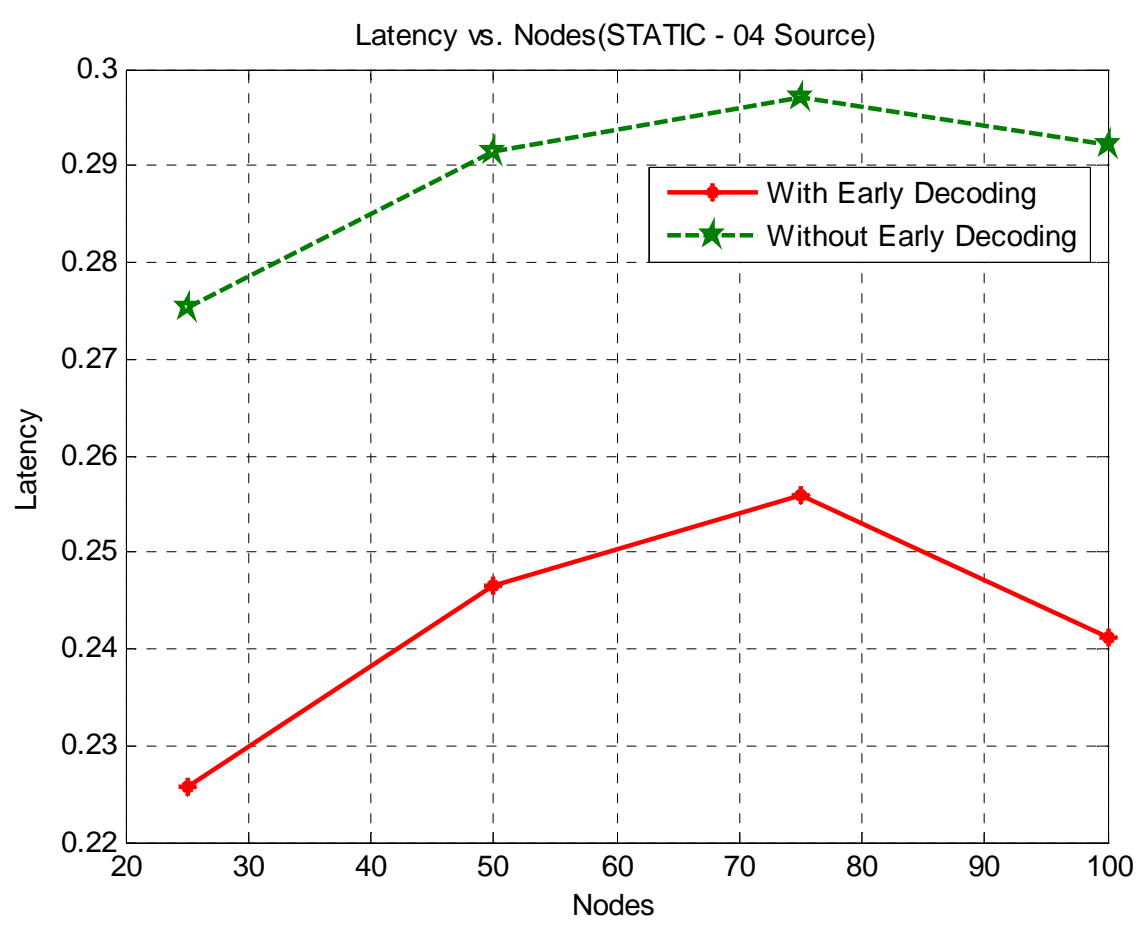

Figure 5.16: Latency vs. Nodes (Static - 04 Sources)

\subsection{Simulation Scenarios}

\subsubsection{Wi-Fi Scenarios}

Our aim in this thesis is to investigate the performance of cross-session generations and to test the adaptability of the protocol. In order to test the adaptability of our protocol to different node densities and data rates, we created different scenarios (static and mobile) with 5, 25, 50, 75 and 100 nodes in a $500 \mathrm{~m} \times 500 \mathrm{~m}$ area for singlesource scenarios, and with $25,50,75$ and 100 nodes in a $500 \mathrm{~m} \times 500 \mathrm{~m}$ for 4 -source scenarios. Figure 5.17 shows some of these scenarios. 

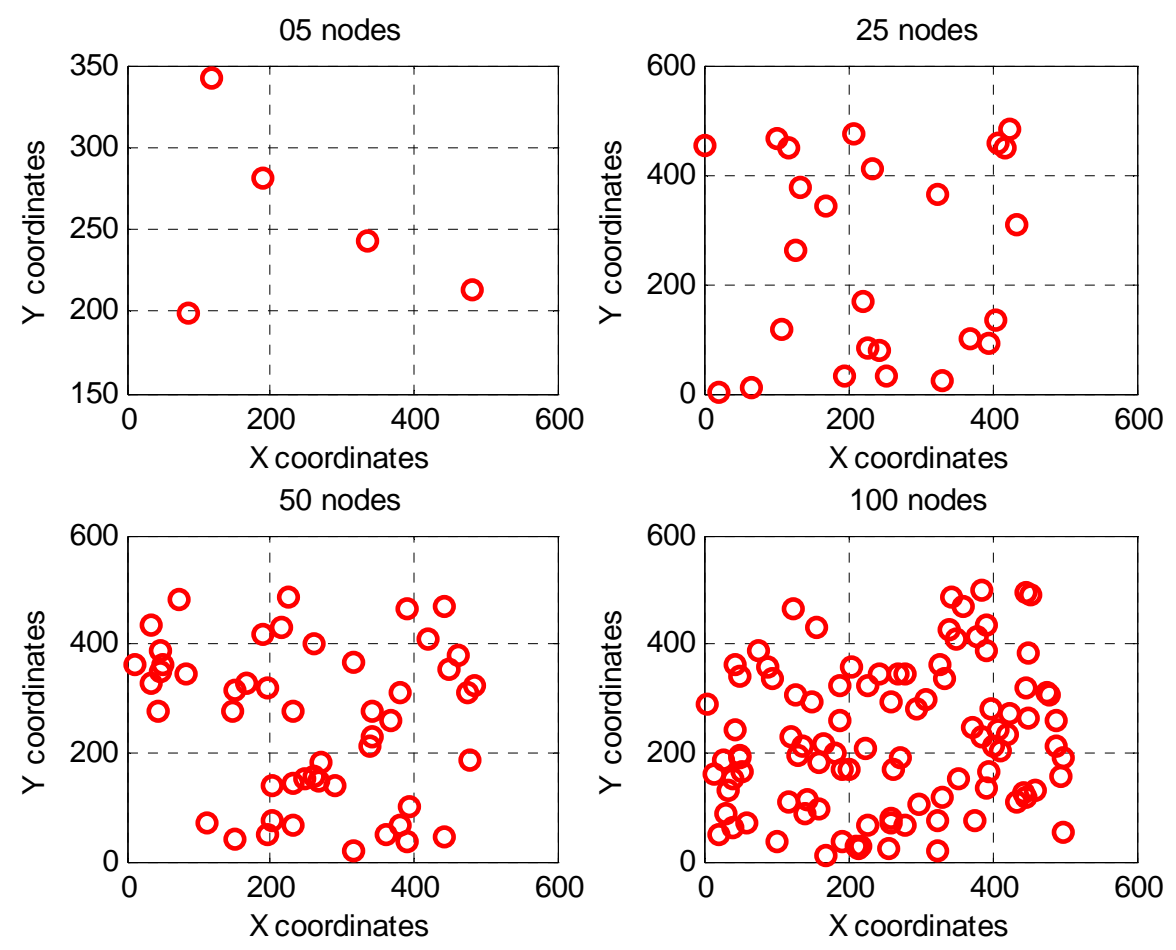

Figure 5.17: Different Scenarios

It can be seen from Figure 5.17 that by increasing the number of nodes in the network in the same area, we are in fact increasing the node density. Fewer retransmissions are required per node in case of a dense network as there are more neighbours available, contributing to the retransmissions. Later, we show through simulations that our protocol is able to appropriately control the number of retransmissions for different scenarios and exhibits steady behavior.

Also, we tested our protocol for different data rates. We selected $1 \mathrm{kbps}, 50 \mathrm{kbps}$ and $100 \mathrm{kbps}$ data rate for single-source scenarios and $1 \mathrm{kbps}, 25 \mathrm{kbps}$ and $50 \mathrm{kbps}$ data rate per source for 4-sources scenarios. Some of the common parameters for each scenario are summarized in Table 5.3. 


\begin{tabular}{|c|c|c|}
\hline \multicolumn{2}{|l|}{ Traffic } & Constant Bit Rate (CBR) \\
\hline \multicolumn{2}{|c|}{ Packet size } & 256 bytes \\
\hline \multicolumn{2}{|l|}{ Area } & $500 \mathrm{~m} \times 500 \mathrm{~m}$ \\
\hline \multicolumn{2}{|c|}{ Propagation model } & 2-ray ground \\
\hline \multicolumn{2}{|c|}{ InterFace Queue (IFQ) } & 50 \\
\hline \multirow{6}{*}{ Mobility } & Model & Random waypoint mobility model \\
\hline & Minimum speed & $2 \mathrm{~m} / \mathrm{s}$ \\
\hline & Maximum speed & $10 \mathrm{~m} / \mathrm{s}$ \\
\hline & Pause & $0 \mathrm{sec}$ \\
\hline & Speed type & Uniform \\
\hline & setdest version & Version 2 \\
\hline
\end{tabular}

Table 5.3: Common Parameters

Based on the results in Section 5.4, we carefully selected the generation size of 6 for single-source scenarios. We did not select a higher value than 6 as it has impact on the complexity of the protocol. Each coded packet carries a source address and sequence number pair to uniquely identify each original packet. A bigger generation size implies each coded packet has to carry more source address and sequence number pairs to uniquely identify each original packet it is carrying, thus increasing the size of the coded packet. We selected a generation size of 4 for multi-source scenarios with timeout value set at 0.1 second. These values are selected based on the sensitivity of our protocol to these values. We selected the optimal values that give the best performance in terms of PDR and latency.

In case of mobility, we used the Random Way-point mobility model with 0 second pause time and minimum and maximum speed of $2 \mathrm{~m} / \mathrm{s}$ and $10 \mathrm{~m} / \mathrm{s}$ respectively. We used the NS2 built-in function SETDEST to generate these scenarios. 


\begin{tabular}{|c|c|c|}
\hline $\begin{array}{l}\text { Generation Size } \\
\text { Number of nodes } \\
\text { Data rate } \\
\text { Gen timeout } \\
\text { GD }\end{array}$ & $\begin{array}{l}06 \\
5-100(\mathrm{Da} \\
1-100 \mathrm{kbps} \\
0.1 \text { second } \\
2\end{array}$ & $\begin{array}{l}\text { Single Source Scenarios } \\
\text { ate fixed at } 50 \mathrm{kbps}) \\
\text { des fixed at } 50 \text { ) }\end{array}$ \\
\hline $\begin{array}{l}\text { Generation Size } \\
\text { Number of nodes } \\
\text { Data rate } \\
\text { Gen timeout } \\
\text { GD }\end{array}$ & $\begin{array}{l}04 \\
25-100(\mathrm{D} \\
1-50 \mathrm{kbps}(\mathrm{I} \\
0.1 \text { second } \\
1\end{array}$ & $\begin{array}{l}\text { 04-Source Scenarios } \\
\text { rate fixed at } 25 \text { kbps per source) } \\
\text { des fixed at 50) }\end{array}$ \\
\hline $\begin{array}{l}\text { Generation Size } \\
\text { Number of nodes } \\
\text { Date Rate } \\
\text { Gen timeout } \\
\text { GD }\end{array}$ & $\begin{array}{l}04 \\
25-100(\mathrm{D} \\
1-50 \mathrm{kbps}( \\
0.1 \text { second } \\
1\end{array}$ & $\begin{array}{l}\text { ource Cross-session Scenarios } \\
\text { rate fixed at } 25 \text { kbps per source) } \\
\text { des fixed at } 50 \text { ) }\end{array}$ \\
\hline $\begin{array}{l}\text { Generation Size } \\
\text { Number of nodes } \\
\text { Gen timeout } \\
\text { GD }\end{array}$ & $\begin{array}{l}04 \\
100 \text { (rate: } 1 \\
0.2 \text { second } \\
1\end{array}$ & $\begin{array}{l}\text { ource Cross-session Scenarios } \\
\text { d } 4 \text { packets /source) }\end{array}$ \\
\hline \multicolumn{2}{|c|}{$\begin{array}{l}\text { Hello interval } \\
\text { (ARLNCCF, SMF and BCAST) }\end{array}$} & $\begin{array}{l}10 \mathrm{sec} \text { for all Static Scenarios } \\
2 \mathrm{sec} \text { for Mobile Scenarios for SMF } \\
\text { and ARLNCCF }\end{array}$ \\
\hline
\end{tabular}

Table 5.4: Simulation Parameters

To test the multi-source scenario performance with and without cross-session generations, we created 4-sources and 100-sources scenarios. We have modified the idea presented in [16] to generate 100 -sources scenario. GD threshold is set to 1 as each node is a source and setting it to higher value will cause the generation size to grow rapidly in 
a dense network of 100 nodes. The simulation is run for 20 second simulation time. Each node generates one packet only for case- 1 and 4 packets for case-2. On average 5 packets are generated per second for case 1 and 20 packets/sec for case 2 from different sources. Different parameters used in various scenarios are summarized in Table 5.4.

\subsubsection{Tactical Scenarios}

We also investigated the protocol performance in tactical scenario [46]. The parameters used for the tactical scenario are given in Table 5.5.

\begin{tabular}{|c|c|c|}
\hline \multicolumn{2}{|l|}{ Traffic } & CBR \\
\hline \multicolumn{2}{|l|}{ Packet size } & 256 bytes \\
\hline \multicolumn{2}{|l|}{ Area } & $40 \mathrm{~km} \times 40 \mathrm{~km}$ \\
\hline \multicolumn{2}{|l|}{ Propagation model } & RiceanShadowing \\
\hline \multicolumn{2}{|l|}{ InterFace Queue } & 50 \\
\hline \multicolumn{2}{|c|}{ Radio transmission range } & $20 \mathrm{~km}$ \\
\hline & \multicolumn{2}{|c|}{ Single Source Tactical Scenarios } \\
\hline Generation Size & \multicolumn{2}{|l|}{06} \\
\hline Number of nodes & \multicolumn{2}{|l|}{50} \\
\hline \multirow[t]{2}{*}{ Data rate } & \multicolumn{2}{|c|}{$1.2 \mathrm{kbps}, 2.4 \mathrm{kbps}, 4.8 \mathrm{kbps}$ (\# of Nodes fixed at 50 ) } \\
\hline & \multicolumn{2}{|c|}{ 04-Source Tactical Scenarios } \\
\hline Generation Size & \multicolumn{2}{|l|}{04} \\
\hline Number of nodes & \multicolumn{2}{|l|}{50} \\
\hline Data rate & \multicolumn{2}{|c|}{$1.2 \mathrm{kbps}, 2.4 \mathrm{kbps}, 4.8 \mathrm{kbps}$ (\# of Nodes fixed at 50 ) } \\
\hline
\end{tabular}

Table 5.5: Simulation Parameters

We used a Ricean Rescue model using the more realistic radio link model based on Ricean fading [47]. 50 nodes are placed in a $40 \mathrm{~km} \times 40 \mathrm{~km}$ area. Bandwidth is lowered 
to $128 \mathrm{kbps}$ and the radio transmission range is set to $20 \mathrm{~km}$. The carrier sense range is set to the transmission range. SMF and simple flooding are selected for comparison. We tested the protocols for both static and mobile scenarios.

\section{Mobility in tactical scenarios [46]}

Some nodes in the tactical scenario move according to the Random Waypoint mobility model and some according to Reference Point Group Mobility Model, but their velocity depends on the group a node belongs to. Of the 50 nodes, 3 nodes, representing command-and-control centers, are nearly stationary. Seven nodes move individually around the whole simulation area based on the Random Waypoint mobility model, with speed randomly selected between $30 \mathrm{~km} / \mathrm{h}$ and $70 \mathrm{~km} / \mathrm{h}$ and 0 pause time. The remaining 40 nodes are grouped into 4 sets of ten nodes each, moving as a group. Each group of 10 nodes moves according to the Reference Point Group Mobility Model, where the reference point moves with a speed randomly selected between 30 and $70 \mathrm{~km} / \mathrm{h}$ and 0 pause time.

Within each group, nodes can deviate from the reference point by $+/-1 \mathrm{~km}$ in each direction. In addition, each of the four groups is assigned to work in one quadrant of the simulation area, with the quadrants slightly overlapping. For example, group one works in the quadrant bounded by $(0,0)$ and $(22 \mathrm{~km}, 22 \mathrm{~km})$; group 2 works in a quadrant bounded by $(0,18 \mathrm{~km})$ and $(18 \mathrm{~km}, 40 \mathrm{~km})$, group three is within the quadrant bounded by $(18 \mathrm{~km}, 0)$ and $(40 \mathrm{~km}, 22 \mathrm{~km})$, and finally group 4 operates within the quadrant bounded by $(18 \mathrm{~km}, 18 \mathrm{~km})$ and $(40 \mathrm{~km}, 40 \mathrm{~km})$. 
In this chapter we have investigated the sensitivity of our protocol to generation timeout and generation size. We also compared the performance with and without early decoding by simulations. We showed that early decoding plays an important role in reducing latency. Similarly, the simulations parameters and scenarios are explained in detail. These scenarios are used to test the adaptive performance as well as the multisource coding feature of our protocol. The results for the above scenarios are discussed in the next two chapters. 


\section{Chapter 6}

\section{Adaptive Performance}

Our main aim in this chapter is to investigate the performance of our protocol by varying the data rate as well as node density. The adaptive performance is analyzed for static, mobile, as well as tactical scenarios. For each scenario, we investigated the performance of our protocol compared to SMF, probabilistic flooding, BCAST and simple flooding. The performance metric selected are PDR, latency and MAC transmissions. First, the results for static scenarios are discussed for 01-source and 04sources. Later on, results for mobile and tactical scenarios are given with explanation.

\subsection{Static Scenarios 01-Source}

Figure 6.1 shows that as the data rate increases, the PDR for simple flooding and BCAST drops significantly. ARLNCCF performs much better than simple flooding, probabilistic flooding and BCAST as the data rate increases. However the PDR performance drops slightly compared to SMF for higher rates. We have calculated $95 \%$ confidence interval for all PDR plots where the difference between ARLNCCF and SMF is less obvious. Further explanation about confidence intervals is provided in Section 6.7. We also note that due to the generation timeout concept in our protocol, which is set at 0.1 second, there is always an inherent delay which is obvious in Figure 6.2. For lower data rates, the delay is around 0.18 second which slightly increases to 0.25 second for our protocol. Probabilistic flooding and SMF induce less delay compared to other protocols. 
SMF shows the best performance in terms of PDR, low latency and fewer MAC transmissions.

When comparing MAC transmissions, we need to explain the trend in more detail. Figure 6.3 show that BCAST has fewer MAC transmissions than ARLNCCF and SMF. However, when we compare the PDR for BCAST in Figure 6.1, it is much poorer than SMF and ARLNCCF. The reason for the low number of MAC transmissions for BCAST is due to packet drops in the InterFace Queue (IFQ) of the source itself. Due to channel unavailability, the protocol drops the source packets at the queue and does not attempt to deliver them. As a result there are fewer MAC transmissions but very low PDR as well. Packet drops for the different protocols are shown in Figure 6.4.

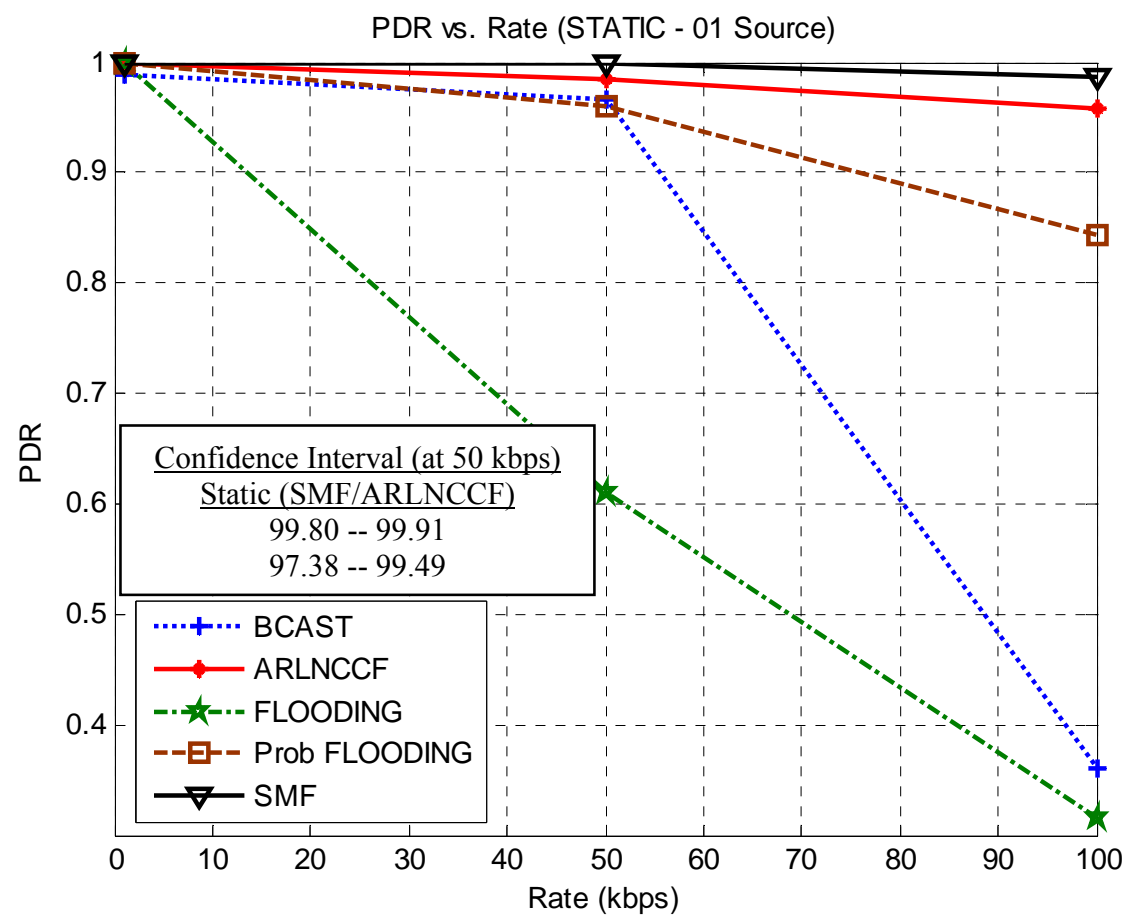

Figure 6.1: PDR vs. Rate (kbps) - (Static - 01 Source) 


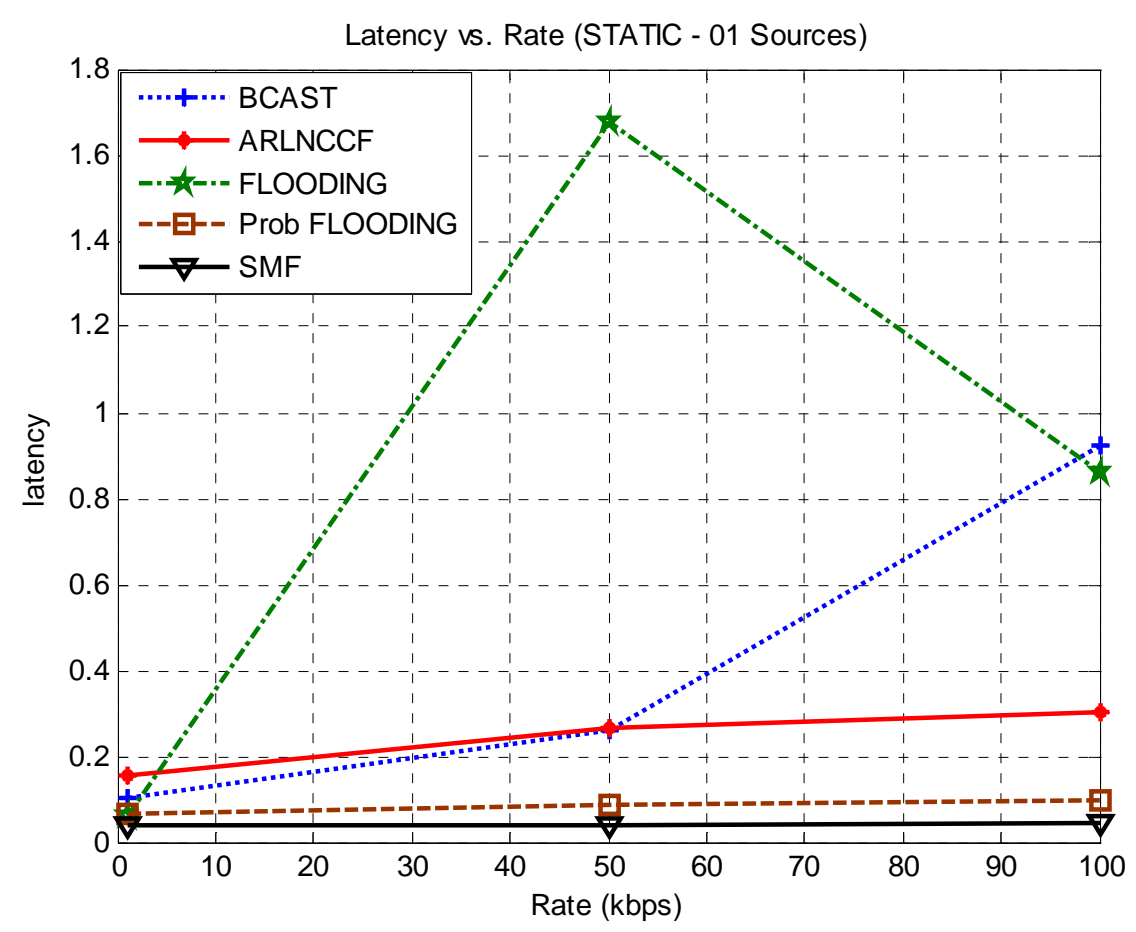

Figure 6.2: Latency vs. Rate (kbps) - (Static - 01 Source)

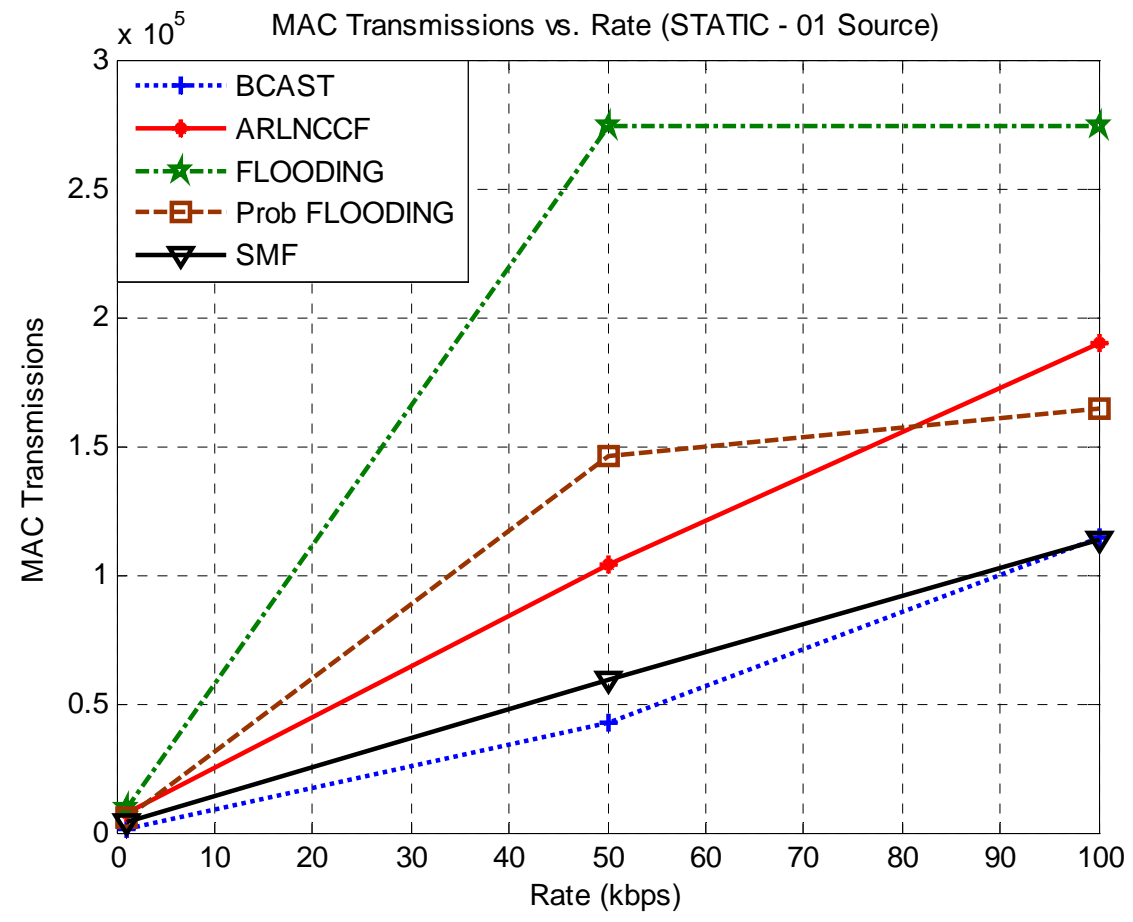

Figure 6.3: MAC Transmissions vs. Rate (kbps) - (Static - 01 Source) 


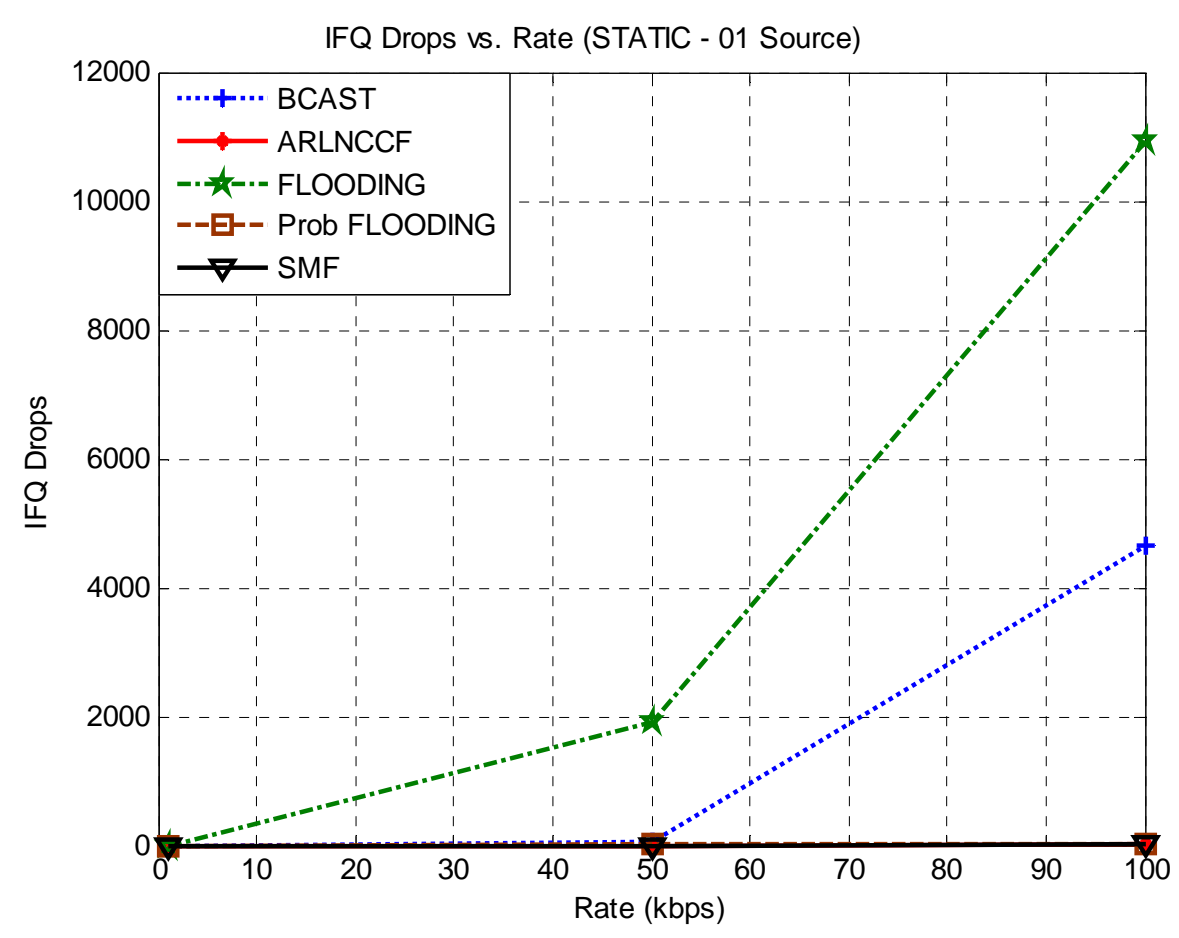

Figure 6.4: IFQ Drops vs. Rate (kbps) - (Static - 01 Source)

Overall, comparison for the single source static scenario shows that SMF and ARLNCCF achieve much higher PDR compared to other protocols with very few MAC drops. Probabilistic flooding is better than BCAST and simple flooding but the optimal probability value for retransmission needs to be determined for each scenario based on different data rate and number of nodes, which is not a practical approach. The optimal probability values vary from $0.5-0.1$ for data rates of $1 \mathrm{kbps}$ to $100 \mathrm{kbps}$ respectively. Similarly as we increase the node densities, a lower probability of retransmission is required to achieve maximum performance.

In Figures 6.5 to 6.8 , the number of nodes is varied from 5 to 100 in the same geographical area. Generation timeout is set to 0.1 second and generation size is 6 for ARLNCCF for single-source scenarios. It is observed that ARLNCCF shows steady performance for all node densities by controlling the number of retransmissions. The 
PDR performance is best for SMF and then ARLNCCF. The latency is around 0.23 seconds for ARLNCCF, which also remains steady for different node densities as shown in Figure 6.6. Due to the generation timeout value in our protocol, the latency is higher compared to SMF and probabilistic flooding. Referring to Figure 6.7 and 6.8 , a trend similar to the one when varying the data rate discussed above is seen for the MAC transmissions and IFQ drops. BCAST and simple flooding have the highest IFQ drop rate. Our protocol shows almost zero IFQ drops, similar to SMF and probabilistic flooding, as shown in Figure 6.8.

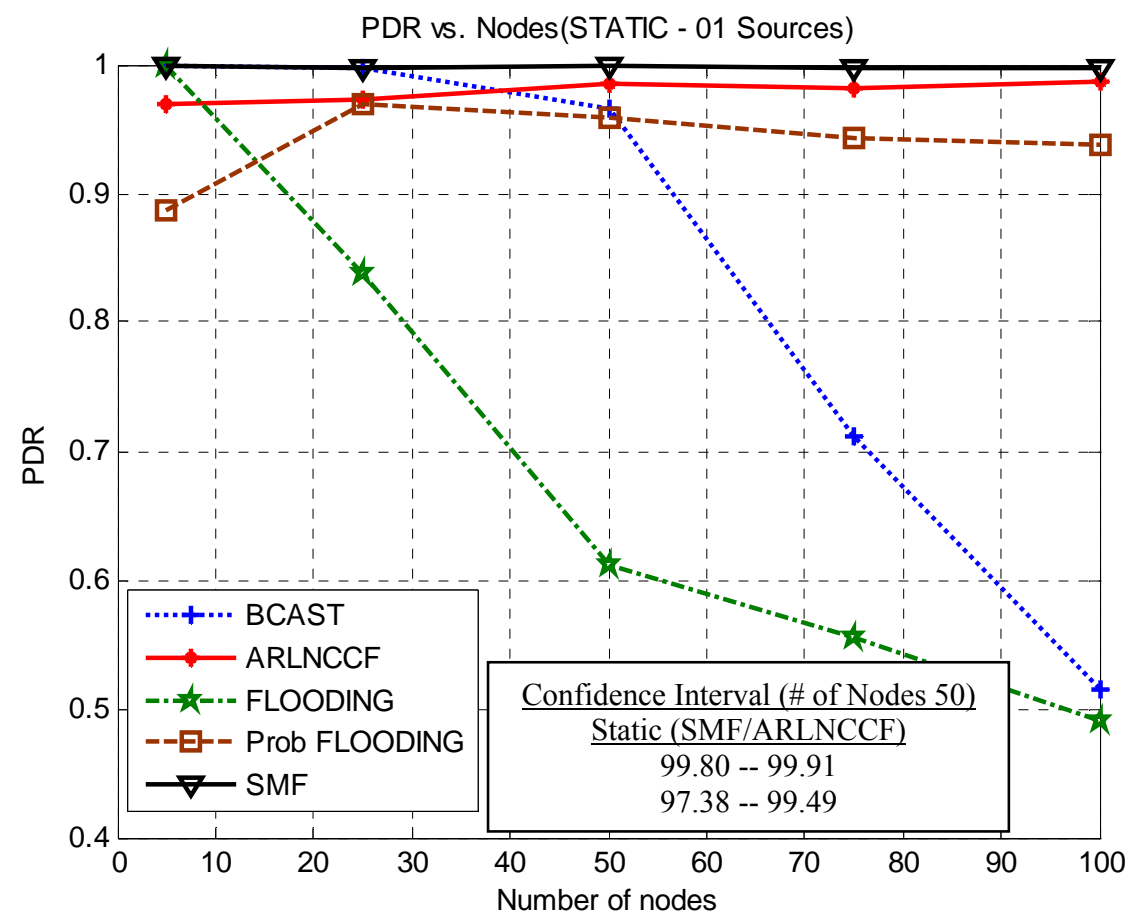

Figure 6.5: PDR vs. Nodes (Static - 01 Source) 


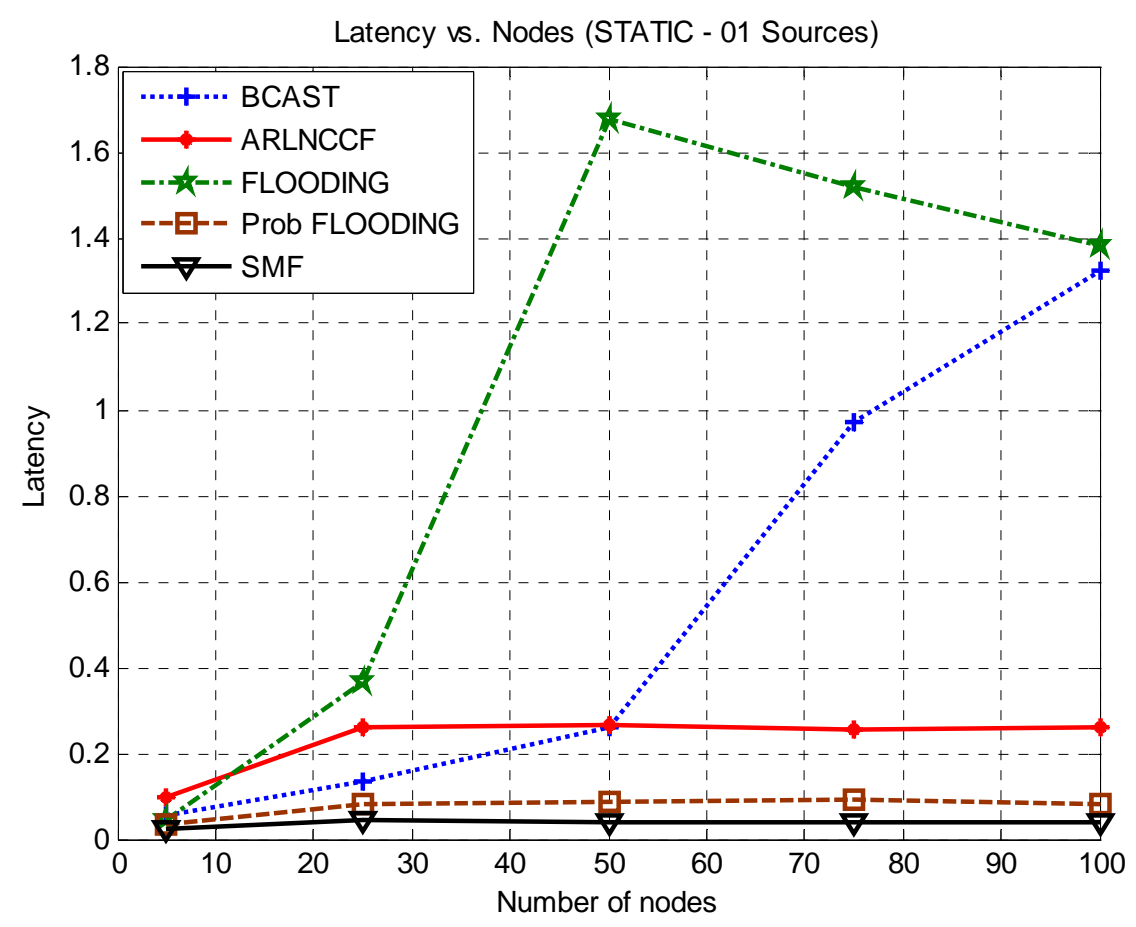

Figure 6.6: Latency vs. Nodes (Static - 01 Source)

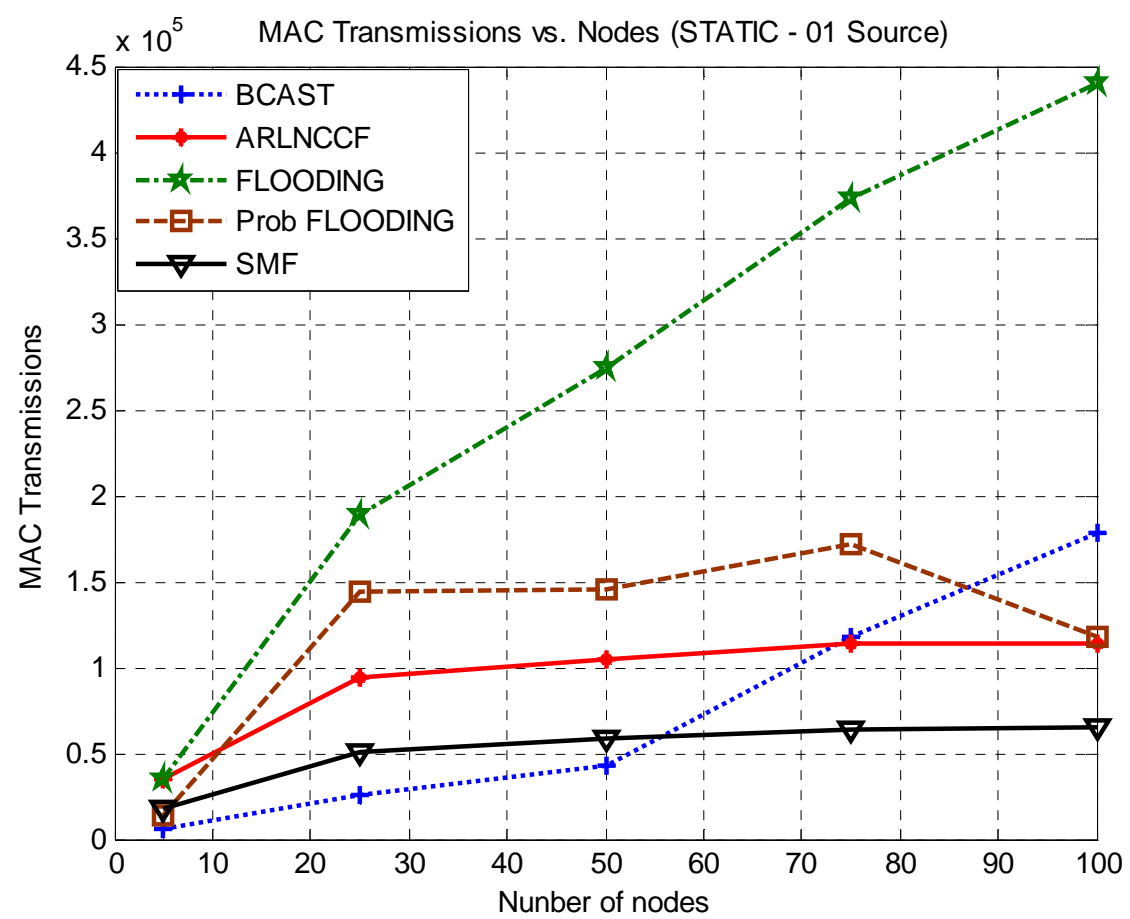

Figure 6.7: MAC Transmissions vs. Nodes (Static - 01 Source) 


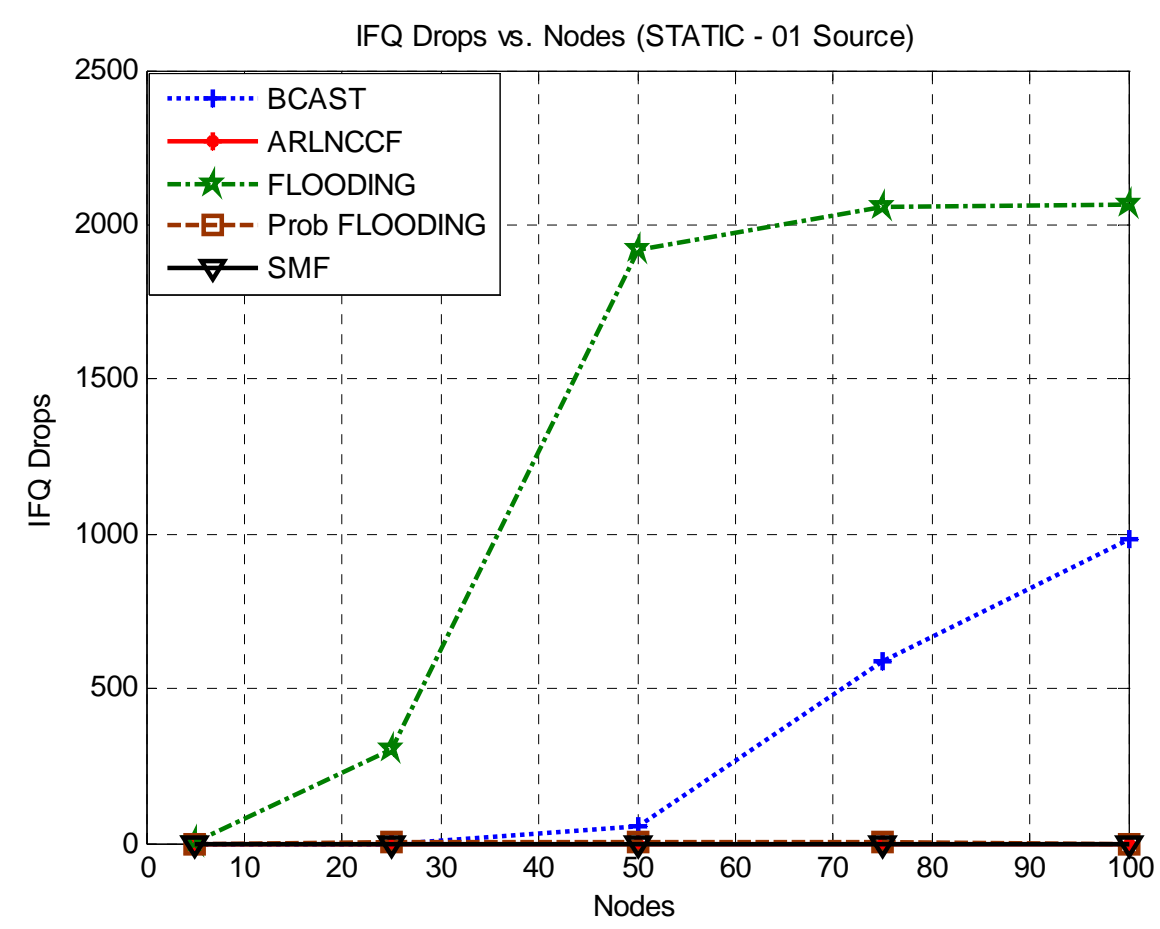

Figure 6.8: IFQ Drops vs. Nodes (Static - 01 Source)

In summary, varying the node density from 5 to 100 , ARLNCCF and SMF show steady performance and there is no effect on the performance of the protocols as the node density increases. For probabilistic flooding, we pre-determined the optimal value of probability of retransmission for different node densities. Still we find its performance in terms of PDR is lower than ARLNCCF and SMF. ARLNCCF and SMF are able to control the number of retransmissions by neighbour knowledge (Although both use different methods to determine the optimal number of required retransmissions), as a result there is no impact observed by varying node densities. Our protocol has an additional delay due to generation timeout but node density has no impact on this delay. 


\subsection{Static Scenarios 04-Sources}

For 4-source static scenarios, generation timeout is set to 0.1 second for a generation size of 4 for ARLNCCF. The PDR and latency performance is consistent with the results obtained for the single source scenarios. As shown in Figure 6.9, at 50kbps per source, the PDR drops to around $83 \%$ for ARLNCCF and around $76 \%$ for SMF. The main reason for this drop is that each source is generating data at the rate of $50 \mathrm{kbps}$ and the accumulative data rate of $200 \mathrm{kbps}$ for 4 sources causes many MAC transmissions, as seen in Figure 6.11. The wireless channel is always occupied and many packets do not get the chance to be transmitted and are dropped at the InterFace Queue (IFQ) of the source node as shown in Figure 6.12. SMF has the minimum latency, which increases to around 0.2 seconds as the data rate increases as shown in Figure 6.10. Our protocol has a latency of around 0.24 seconds, which remains consistent as the data rate increases.

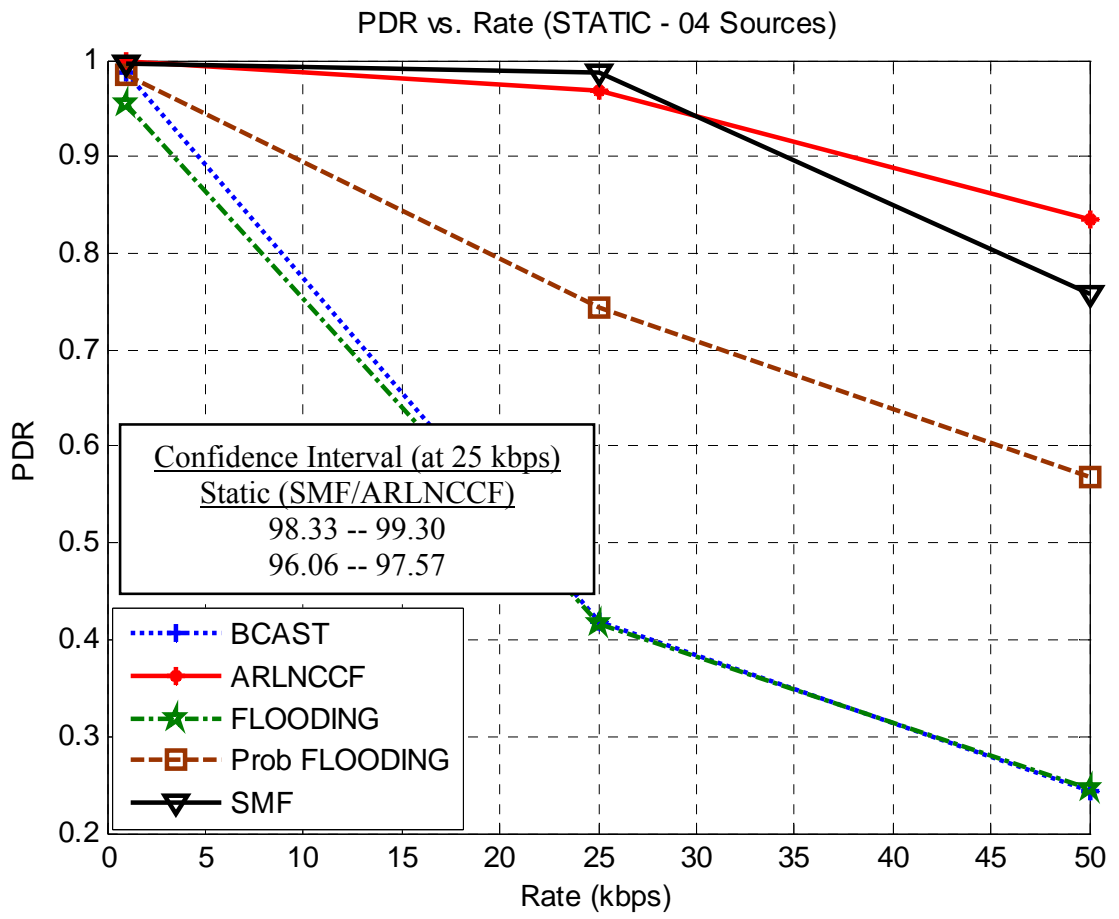

Figure 6.9: PDR vs. Rate (kbps) - (Static - 04 Sources) 


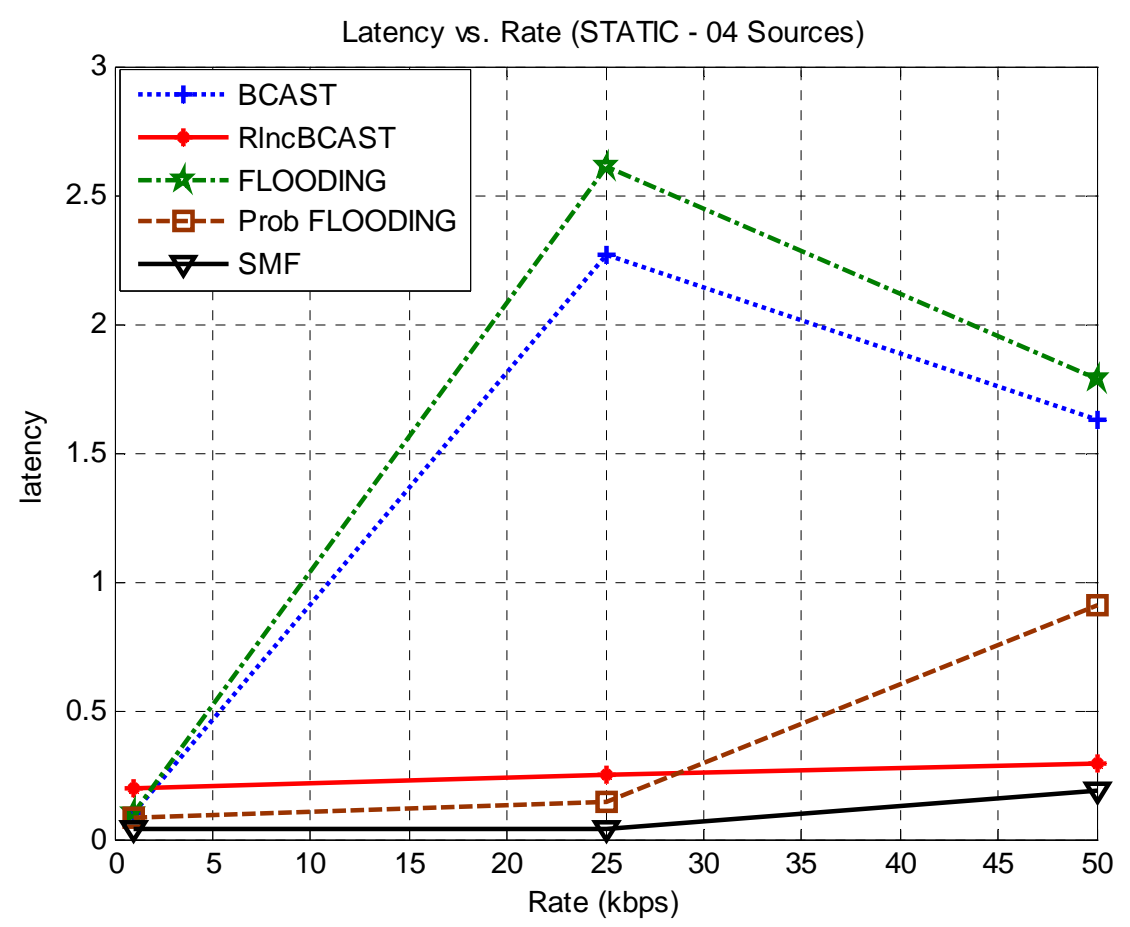

Figure 6.10: Latency vs. Rate (kbps) - (Static - 04 Sources)

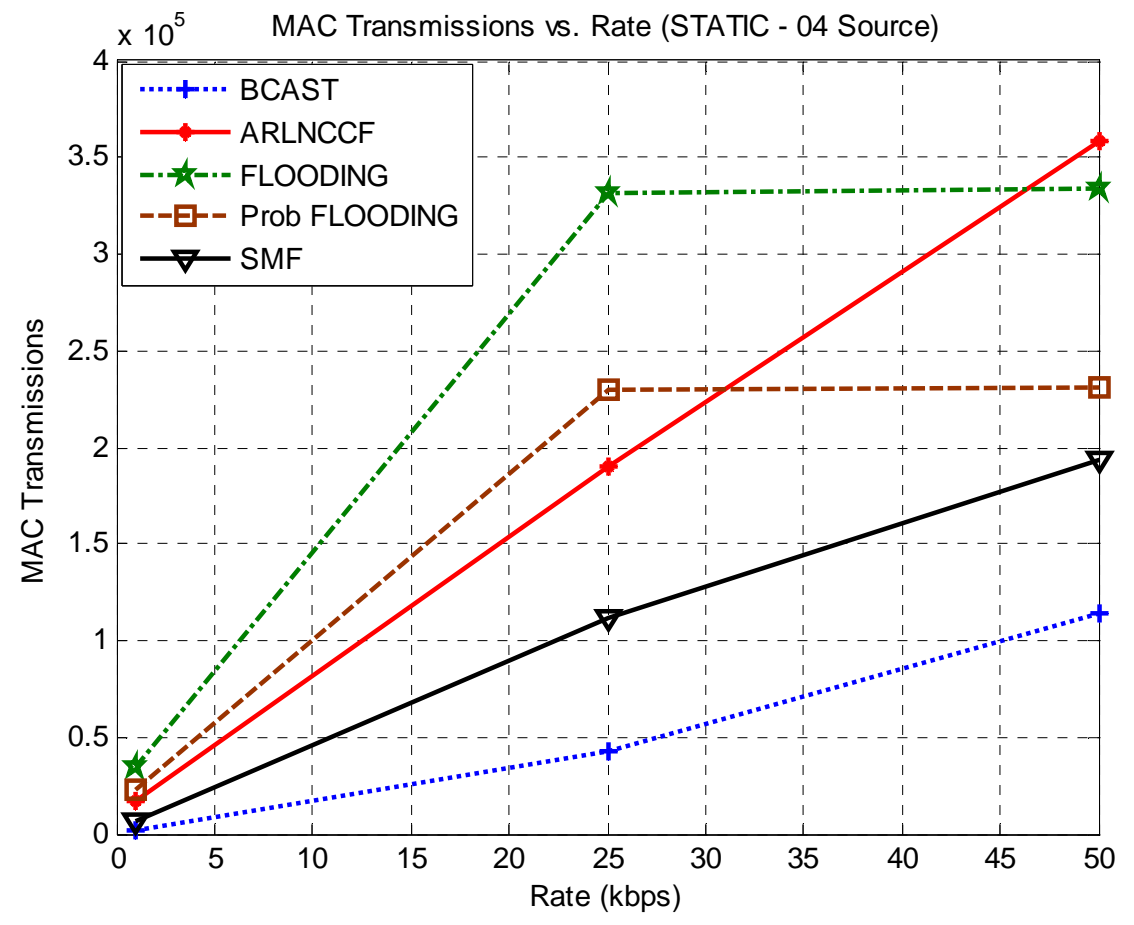

Figure 6.11: MAC Transmissions vs. Rate (kbps) - (Static - 04 Sources) 


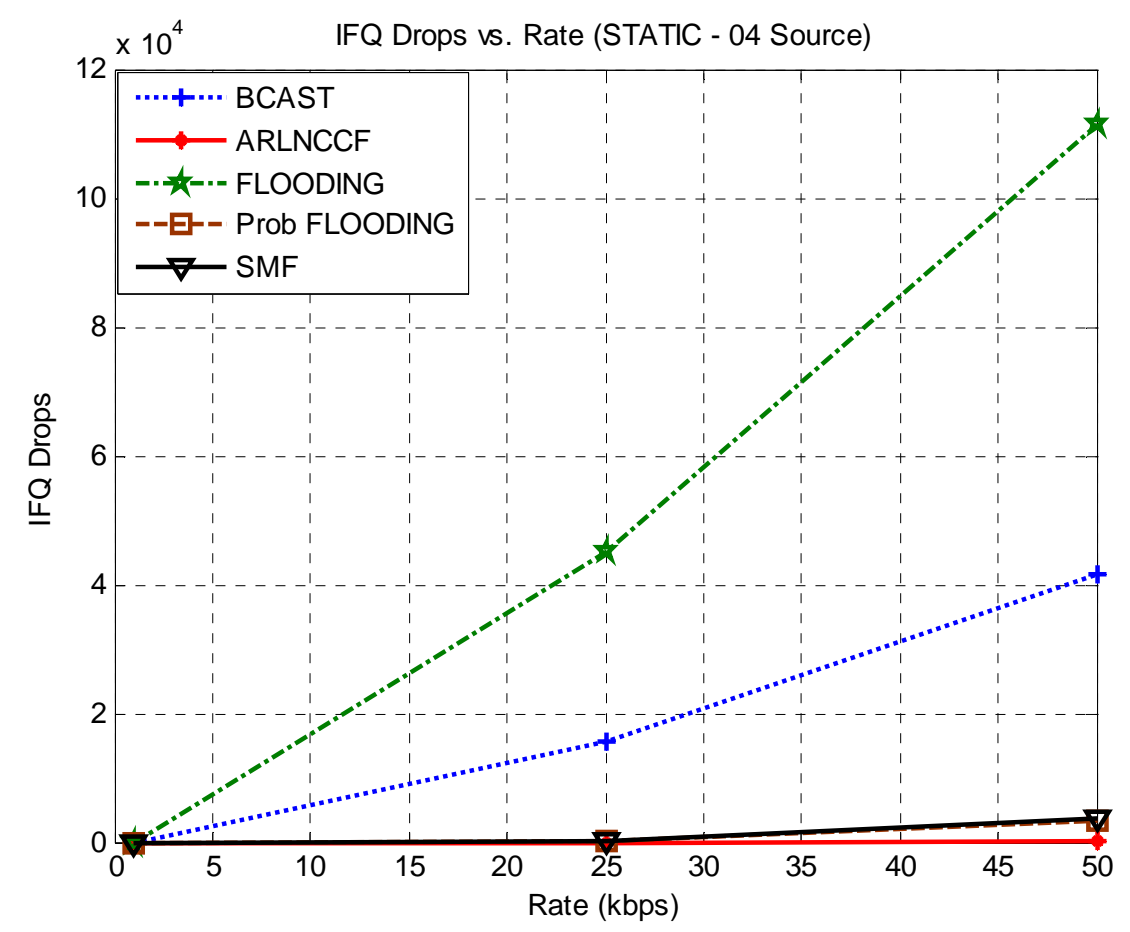

Figure 6.12: IFQ Drops vs. Rate (kbps) - (Static - 04 Sources)

Figure 6.11 shows that BCAST has the lowest number of MAC transmissions but the reason for this is that BCAST does not attempt to deliver most of the packets due to IFQ drops as shown in Figure 6.12. Our protocol has more MAC transmissions for 4 source scenarios for higher data rates with Minimum IFQ drops.

SMF shows the best performance with higher PDR and fewer MAC transmissions with minimum IFQ drops. Our protocol has the best PDR at a data rate of $50 \mathrm{kbps}$ per source but at the cost of a higher number of MAC transmissions. 
The next series of results from Figure 6.13 to 6.16 are for 4-source static scenarios when varying the number of nodes and hence the network density. The rate is fixed at $25 \mathrm{kbps}$ per source. The generation timeout is 0.1 second with a generation size set to 4 . The number of nodes is increased from 25 to 100 in the same geographical area. SMF and ARLNCCF have the best PDR but SMF achieves a slightly higher PDR with fewer MAC transmissions. Latency for ARLNCCF increases from 0.18 second for 25 nodes to 0.21 second for 100 node scenarios.

The overall performance of ARLNCCF for static scenarios is that it shows steady performance for both 01-source and 04-sources. The protocol adapts itself well to different data rates as well as different node densities. However, our protocol achieves this performance at the cost of more number of MAC transmissions compared to SMF, especially for 04 -source scenarios with a data rate of $50 \mathrm{kbps}$ per source.

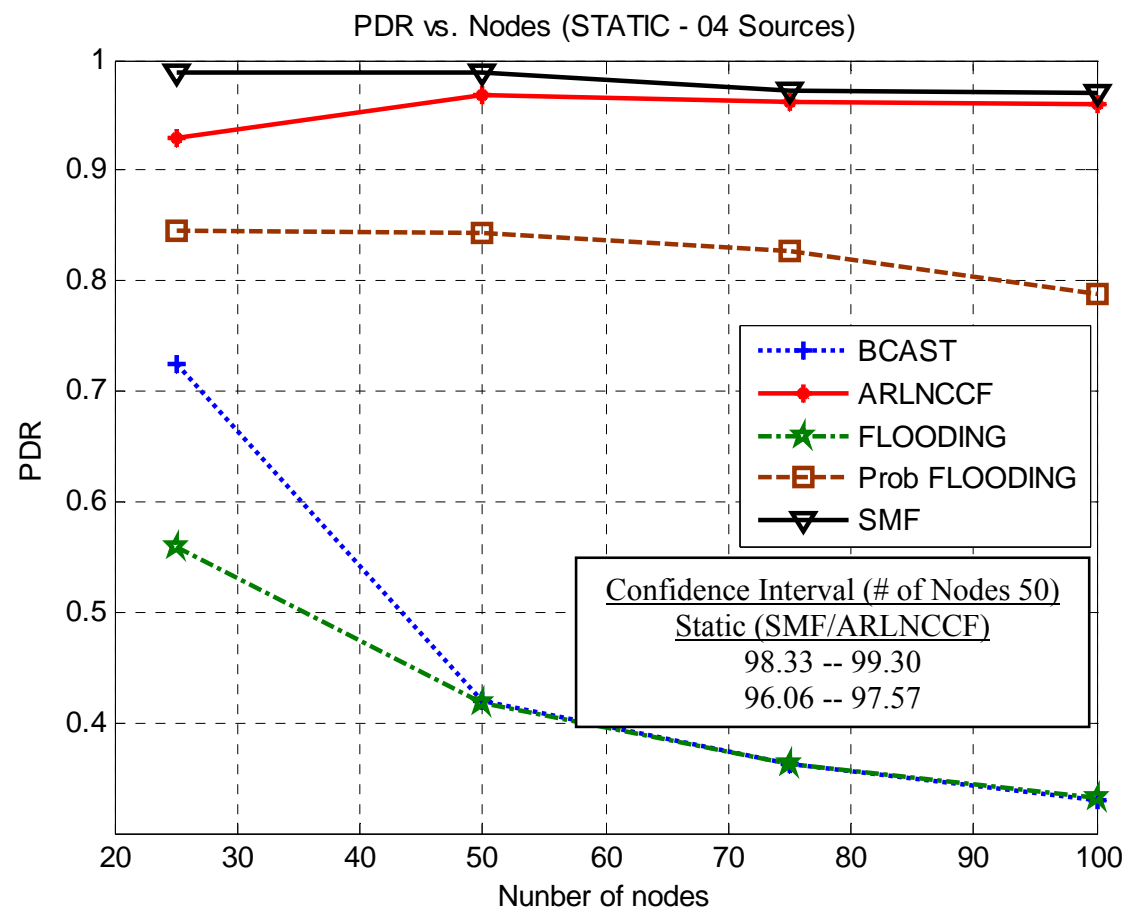

Figure 6.13: PDR vs. Nodes (Static - 04 Sources) 


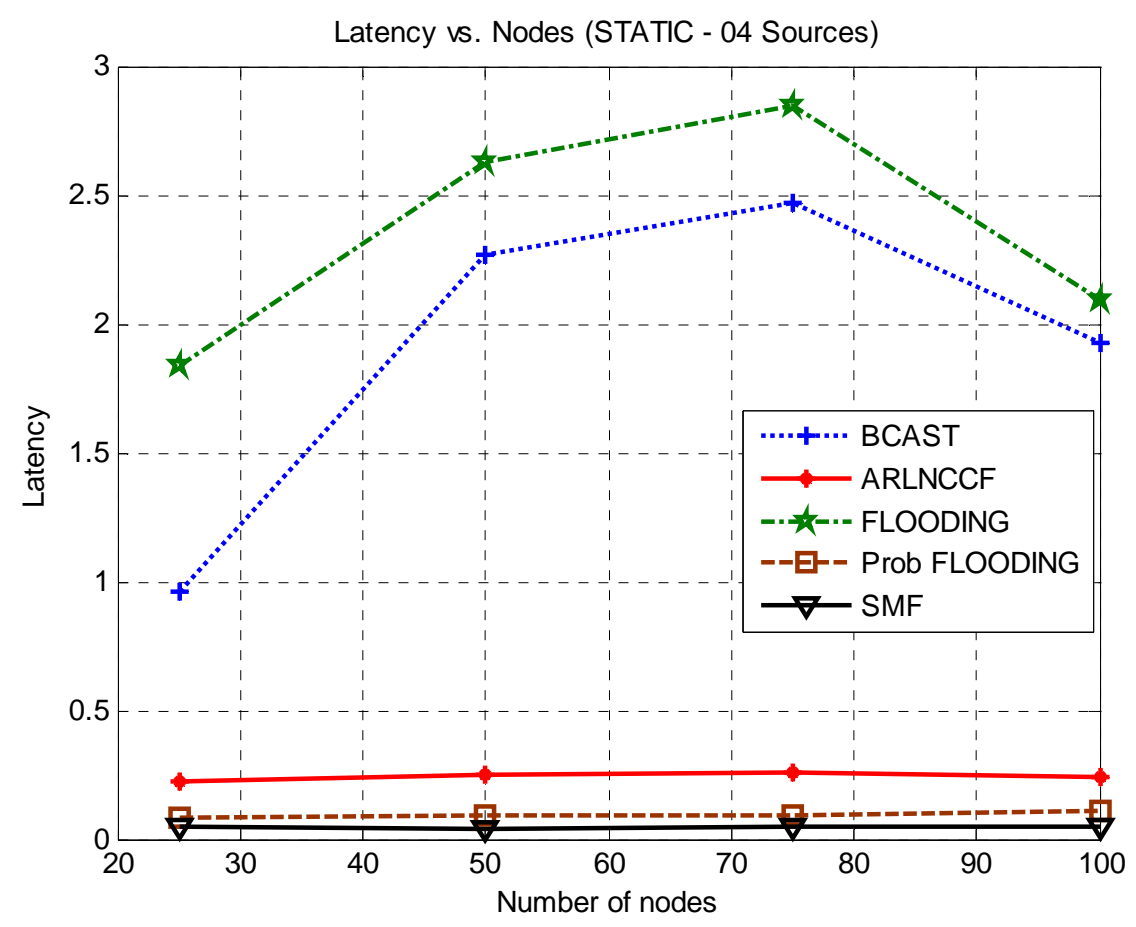

Figure 6.14: Latency vs. Nodes (Static - 04 Sources)

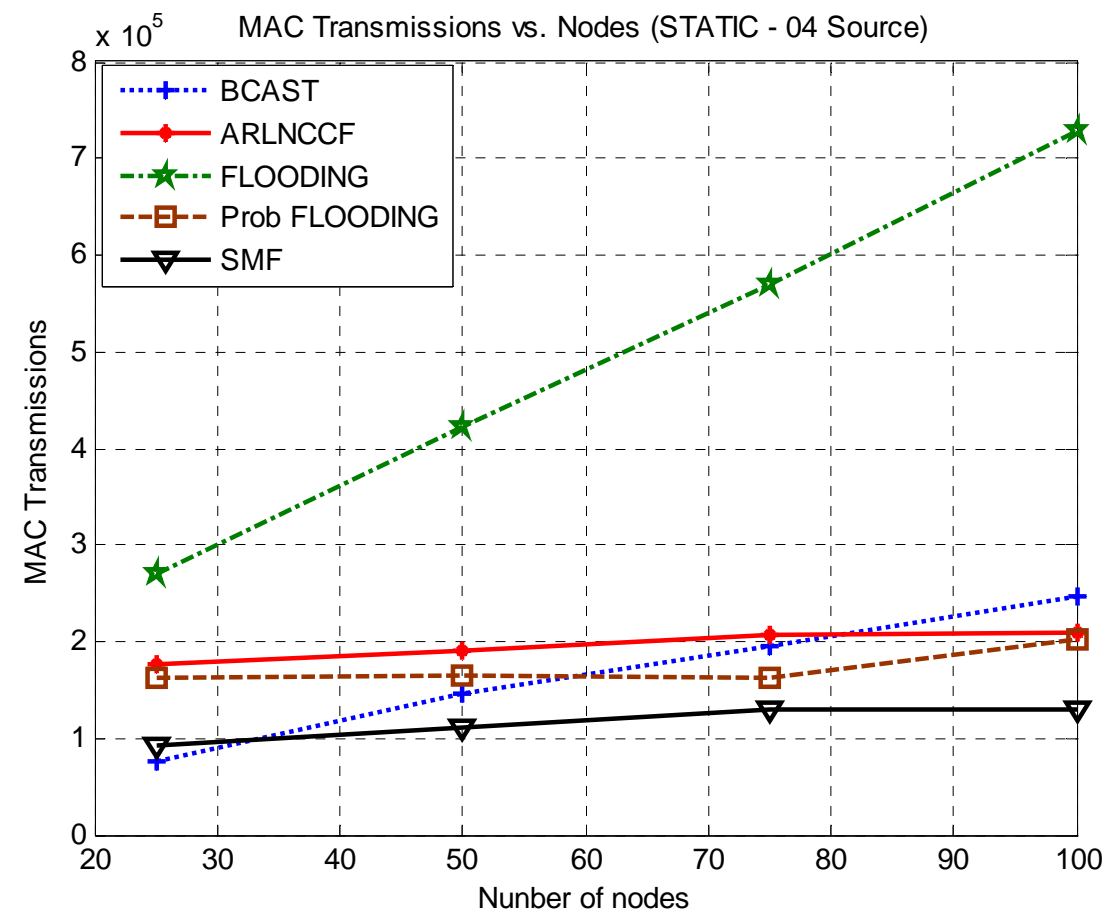

Figure 6.15: MAC Transmissions vs. Nodes (Static - 04 Sources) 


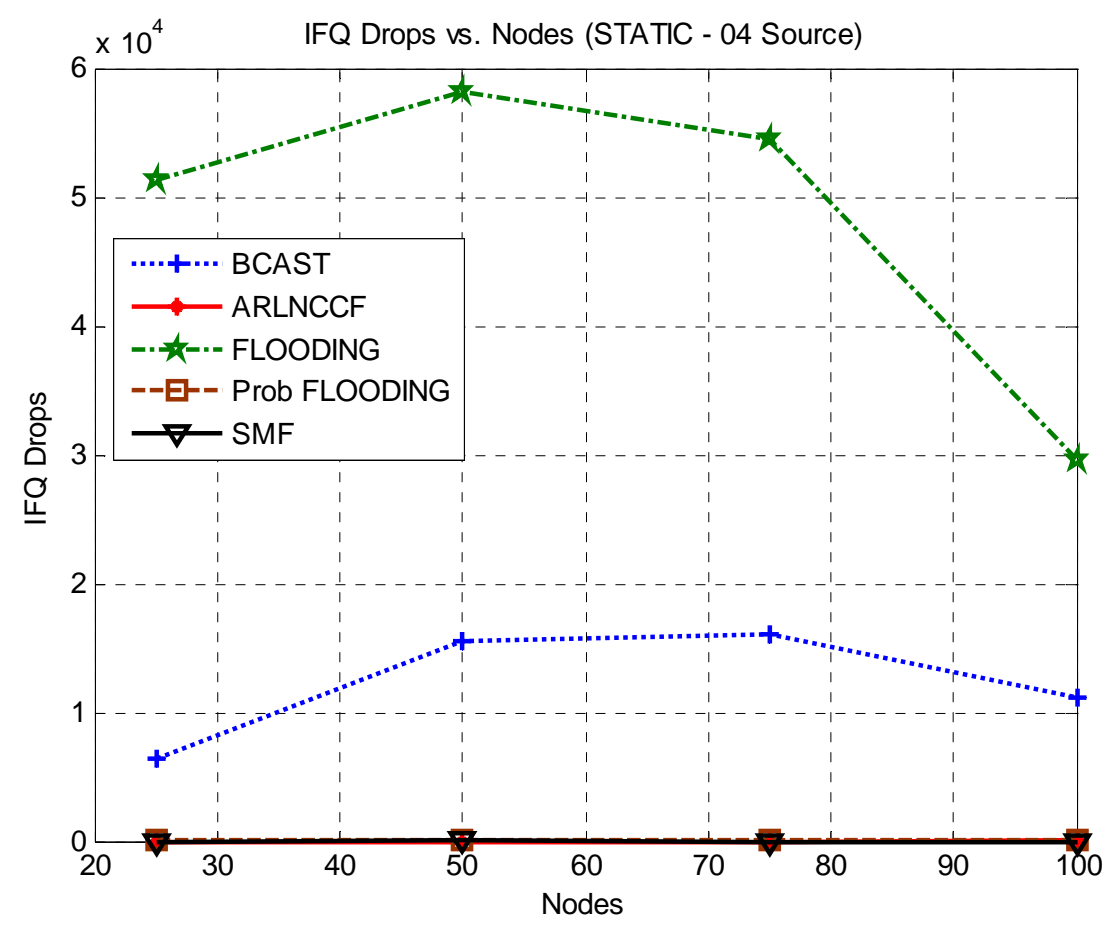

Figure 6.16: IFQ Drops vs. Nodes (Static - 04 Sources)

\subsection{Mobile Scenarios 01-Source}

The next series of results from Figure 6.17 to 6.24 show the results for 01 -source mobile scenarios and results are presented for different data rates and node densities. Generation timeout is set to 0.1 seconds with a generation size of 6 for single-source scenarios for ARLNCCF. SMF and ARLNCCF have the best PDR performance. As shown in Figure 6.17, ARLNCCF performs slightly better than SMF for lower data rates. As the data rate increases, PDR for ARLNCCF starts to decline slightly to around $95 \%$. Similarly, Figure 6.18 shows that latency varies from around 0.16 second to 0.20 second as the data rate increases. 


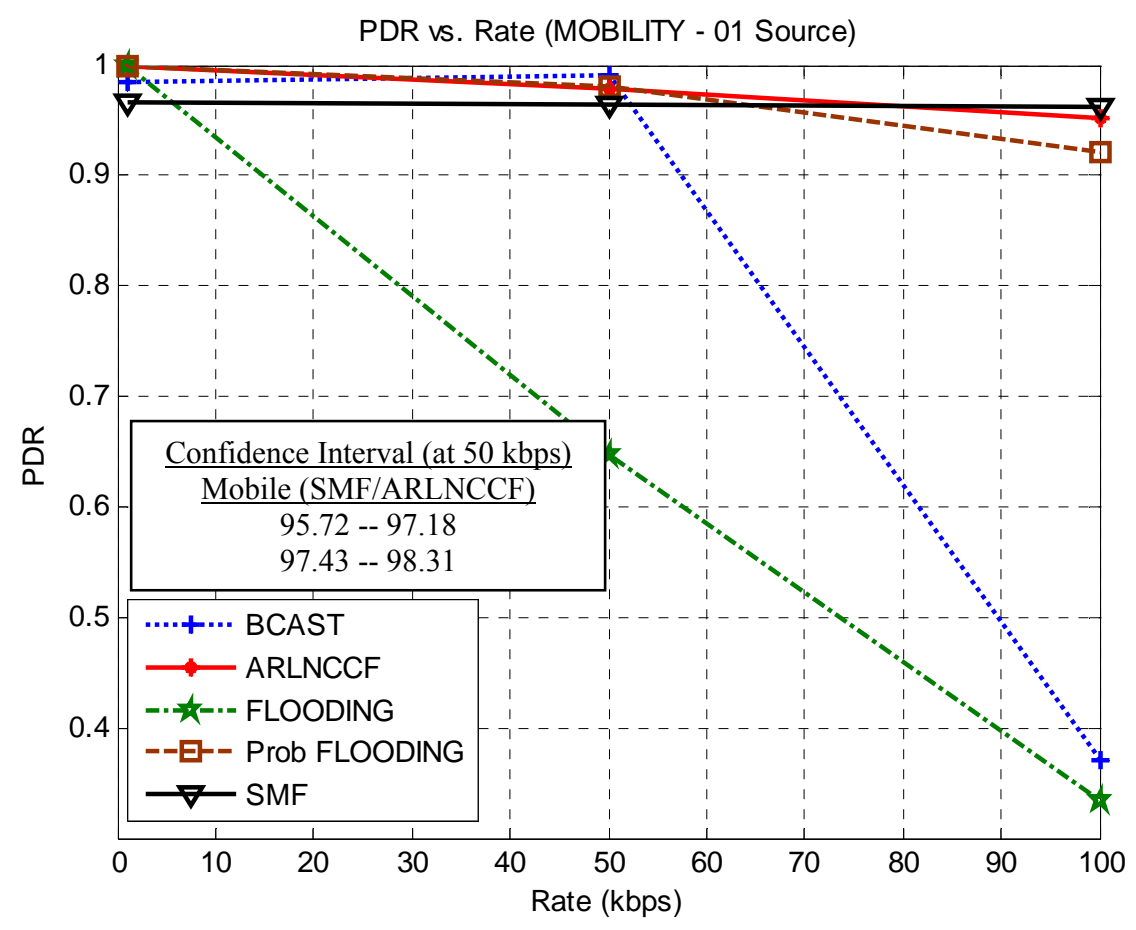

Figure 6.17: PDR vs. Rate (kbps) - (Mobility - 01 Source)



Figure 6.18: Latency vs. Rate (kbps) - (Mobility - 01 Source) 


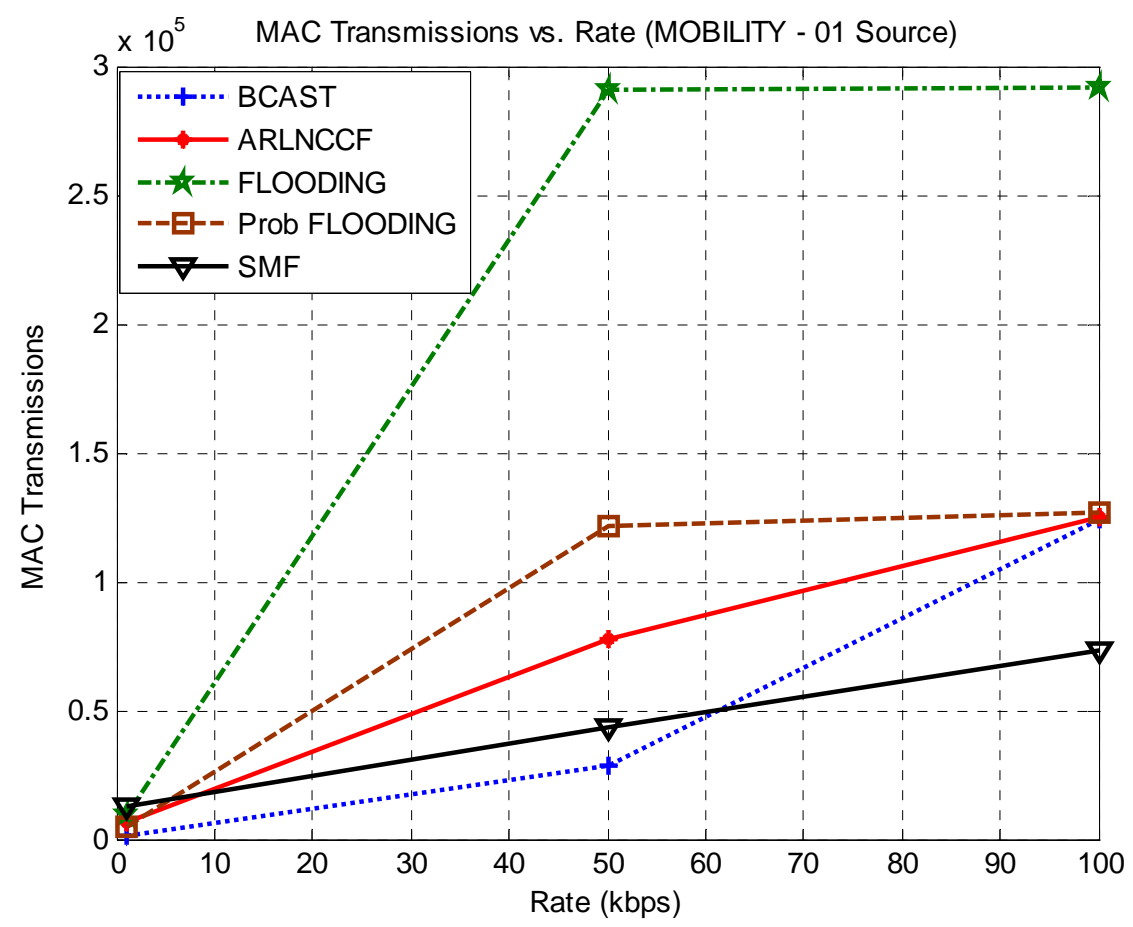

Figure 6.19: MAC Transmissions vs. Rate (kbps) - (Mobility - 01 Source)

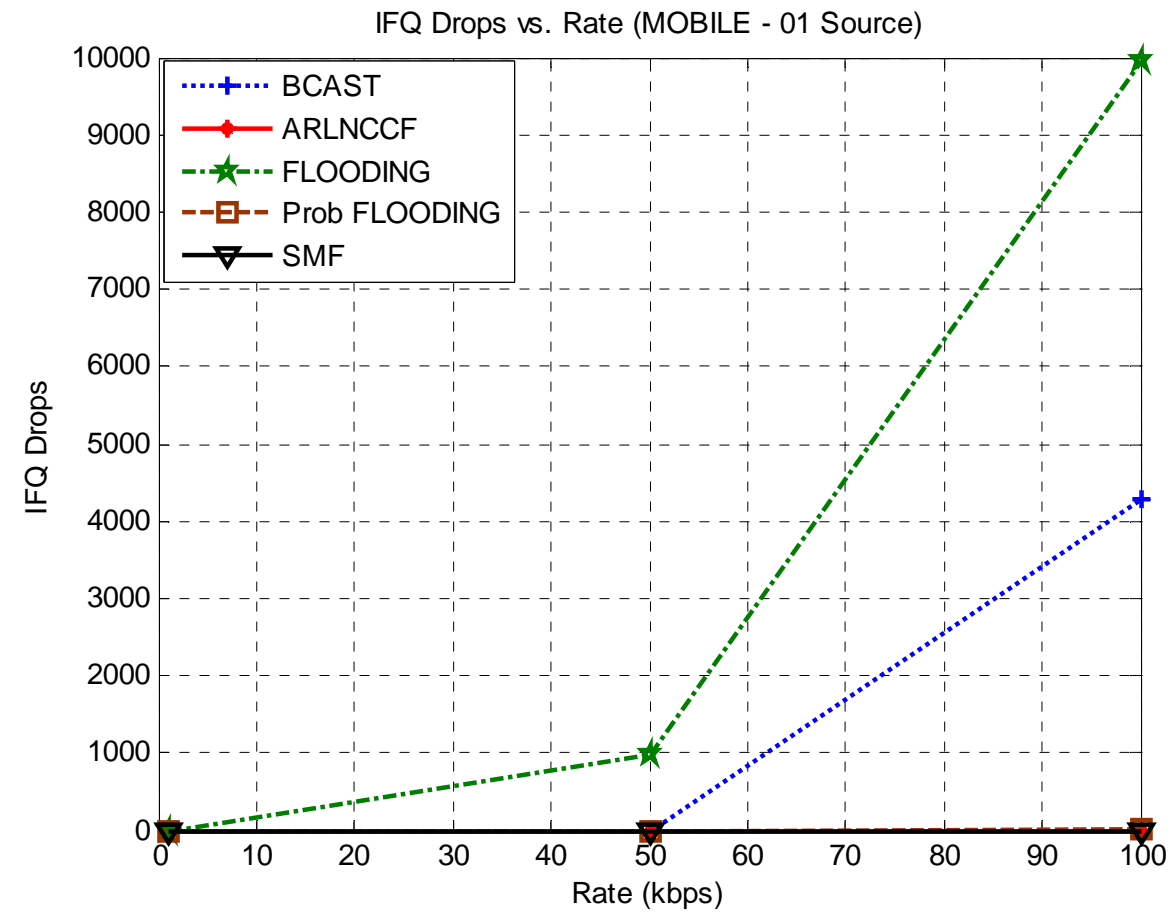

Figure 6.20: IFQ Drops vs. Rate (kbps) - (Mobility - 01 Source) 
Comparing Figure 6.19 to Figure 6.3, we observe that for mobiles scenarios, overall, all protocols require fewer MAC transmissions to achieve the PDR as given in Figure 6.1 and Figure 6.17 respectively. SMF and ARLNCCF have almost zero IFQ drops compared to BCAST and flooding.

The next series of results from Figure 6.21 to Figure 6.24 show the results obtained by varying the node densities. For single source mobile scenarios, we observe that ARLNCCF, SMF and probabilistic flooding show steady performance. However, for probabilistic flooding we need to find the optimal value of probability of retransmissions for each data point. So there is no adaptivity as far as probabilistic flooding is concerned. For number of nodes set to 5 , there is high probability that the network is not fully connected, therefore we get lower PDR for all protocols as shown in Figure 6.21. However, we observe that as the node density increases, the PDR improves.

Latency performance in Figure 6.22 show a similar trend to the results observed before. The same inherent delay is observed due to generation timeout which is set at 0.1 second for ARLNCCF. SMF has the minimum delay. Flooding and BCAST have the worst performance in terms of both PDR and latency as the number of nodes increases.

MAC transmissions for ARLNCCF are higher, as before, compared to SMF. The protocol with the highest number of MAC transmission is simple flooding as shown in Figure 6.23. 


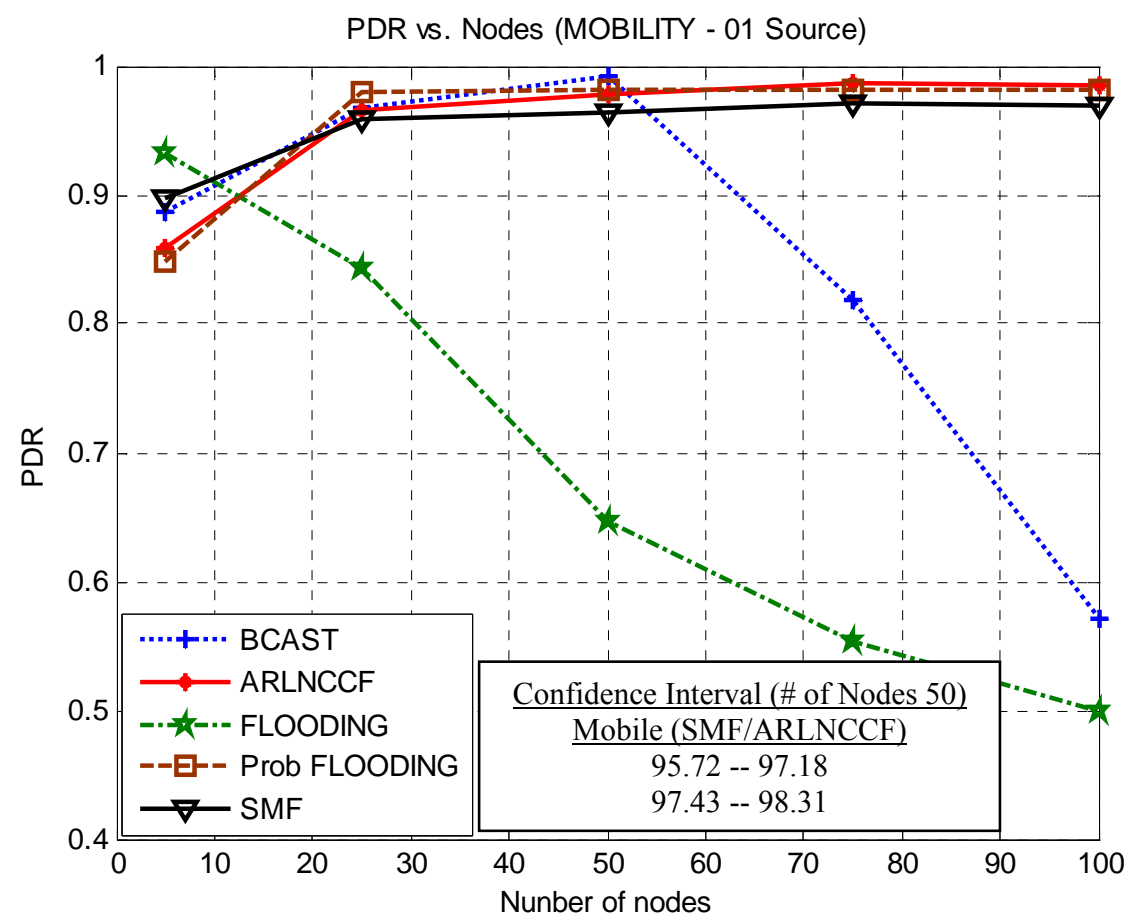

Figure 6.21: PDR vs. Nodes - (Mobility - 01 Source)

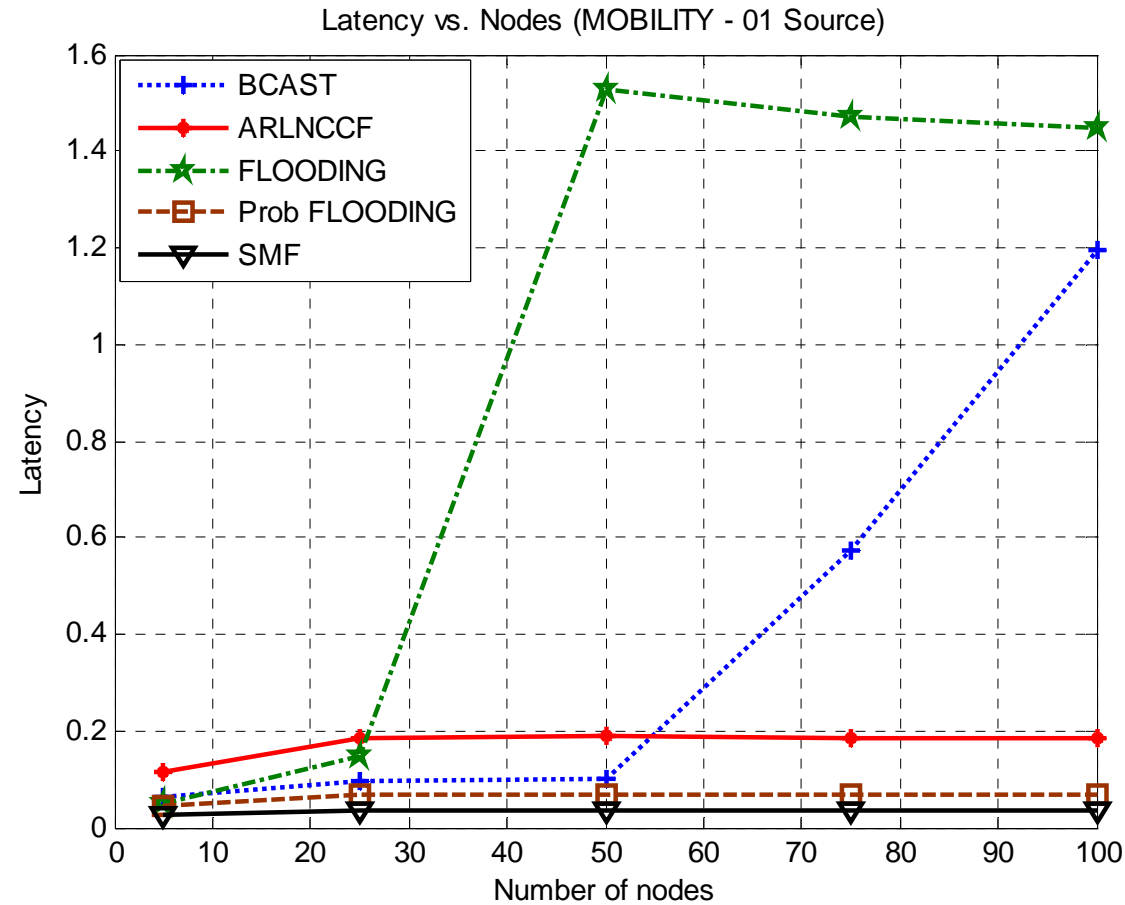

Figure 6.22: Latency vs. Nodes - (Mobility - 01 Source) 


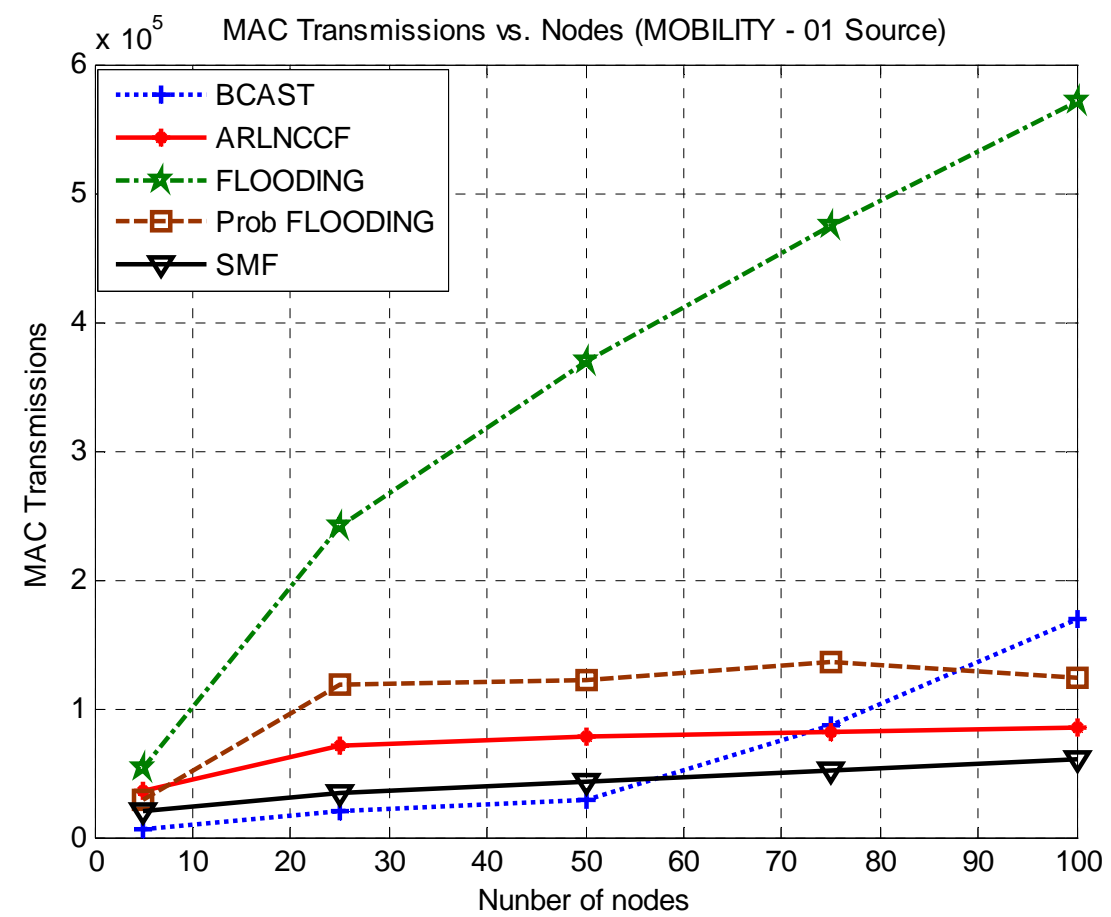

Figure 6.23: MAC Transmissions vs. Nodes - (Mobility - 01 Source)

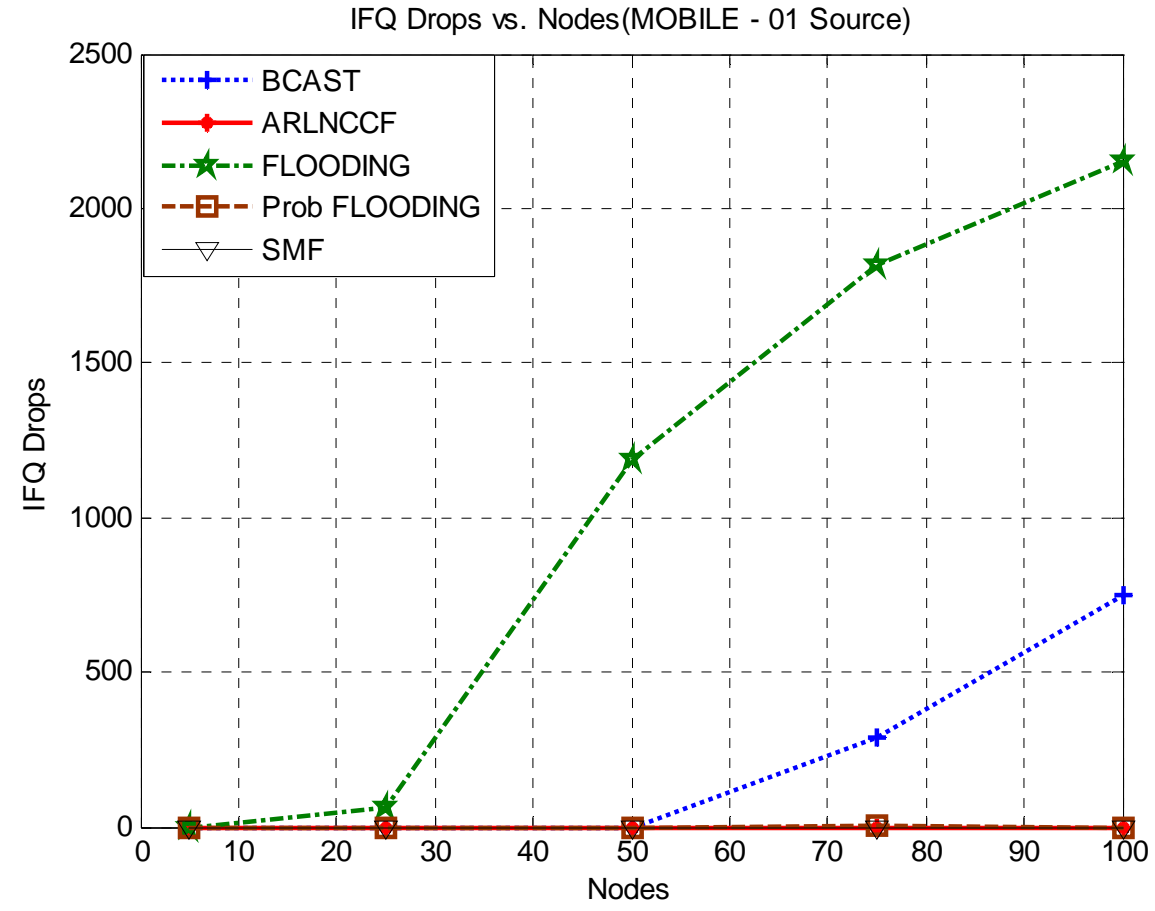

Figure 6.24: IFQ Drops vs. Nodes - (Mobility - 01 Source) 


\subsection{Mobile Scenarios 04-Sources}

Figures 6.25 to 6.32 show the performance for 04 -source scenarios with different data rates and node densities. The PDR for SMF and ARLNCCF are almost the same for higher data rates but ARLNCCF achieves comparable PDR at the cost of a higher number of MAC transmissions. Flooding and BCAST show the worst performance. BCAST, as before, has a large number of IFQ drops which results in fewer MAC transmissions. Similar performance is observed for different node densities.

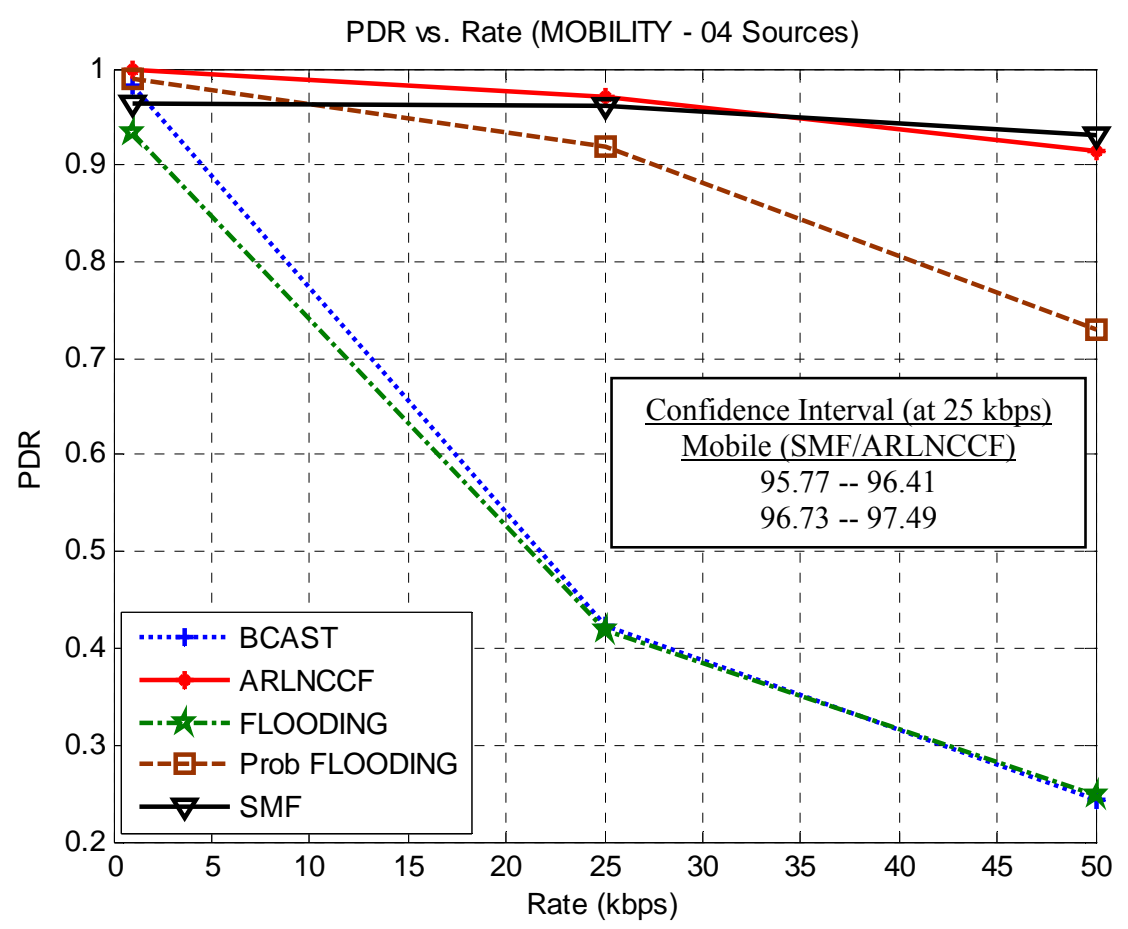

Figure 6.25: PDR vs. Rate (kbps) - (Mobility - 04 Sources) 


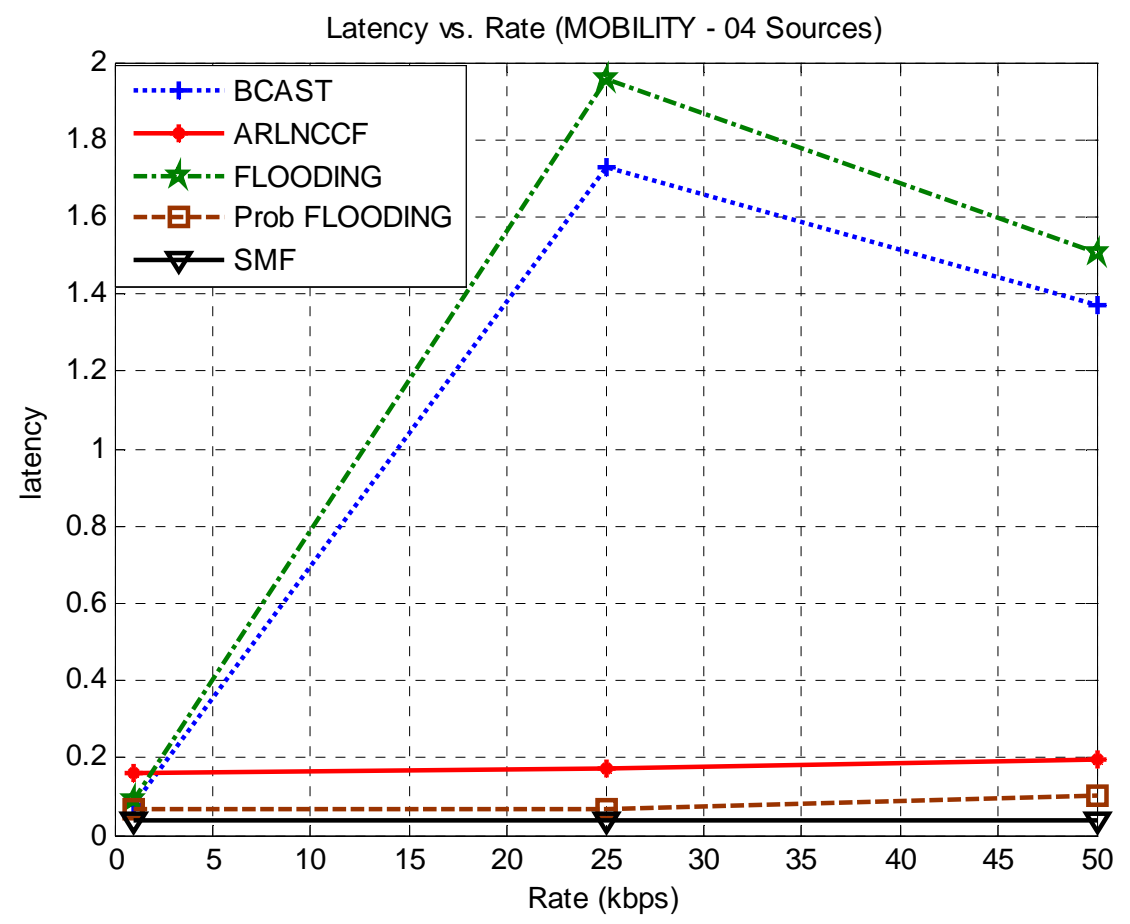

Figure 6.26: Latency vs. Rate (kbps) - (Mobility - 04 Sources)

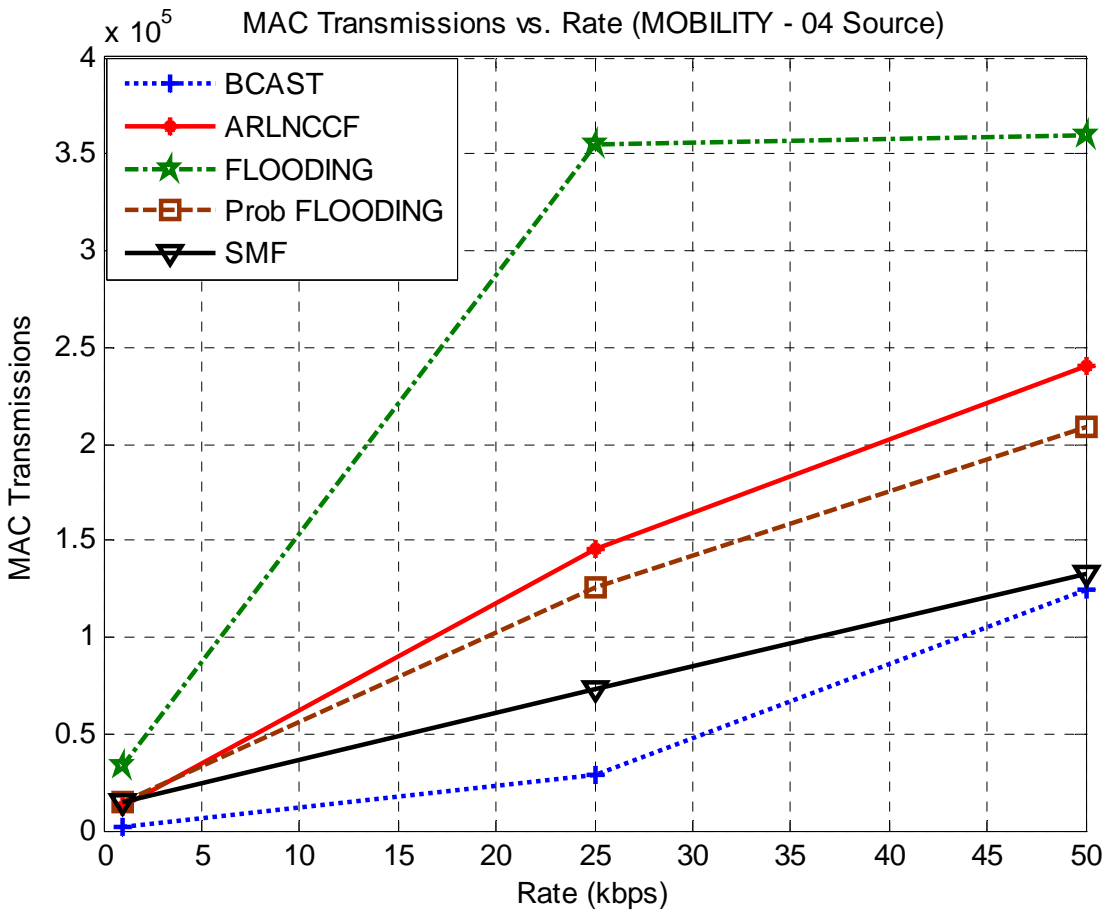

Figure 6.27: MAC Transmissions vs. Rate (kbps) - (Mobility - 04 Sources) 


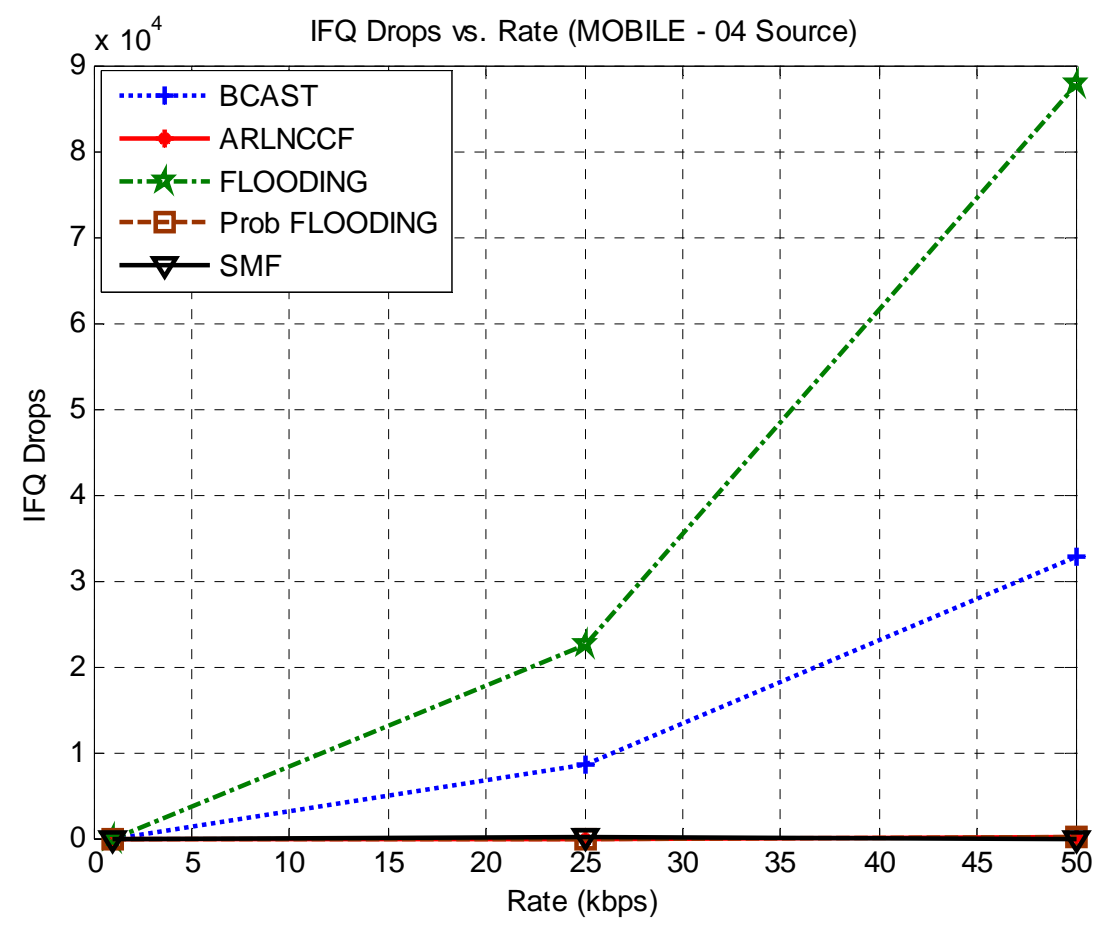

Figure 6.28: IFQ Drops vs. Rate (kbps) - (Mobility - 04 Sources)

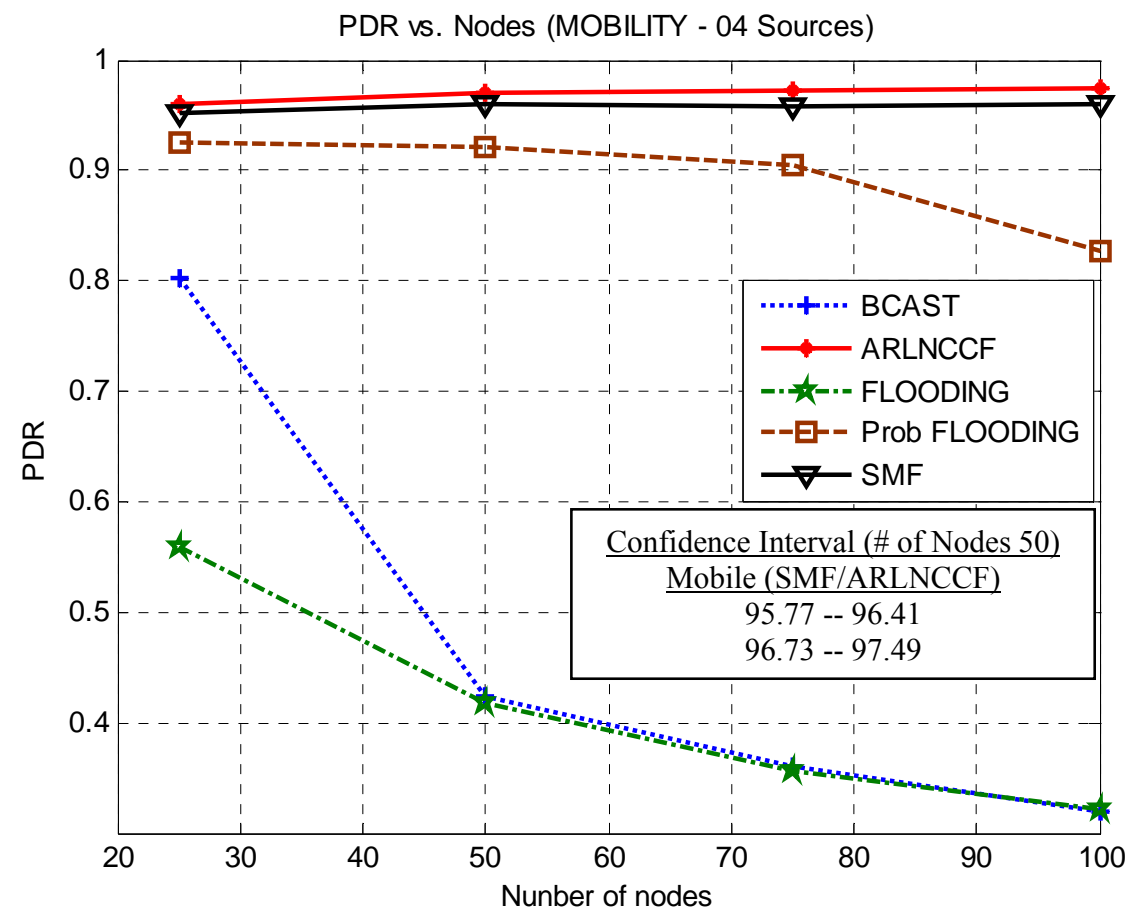

Figure 6.29: PDR vs. Nodes - (Mobility - 04 Sources) 


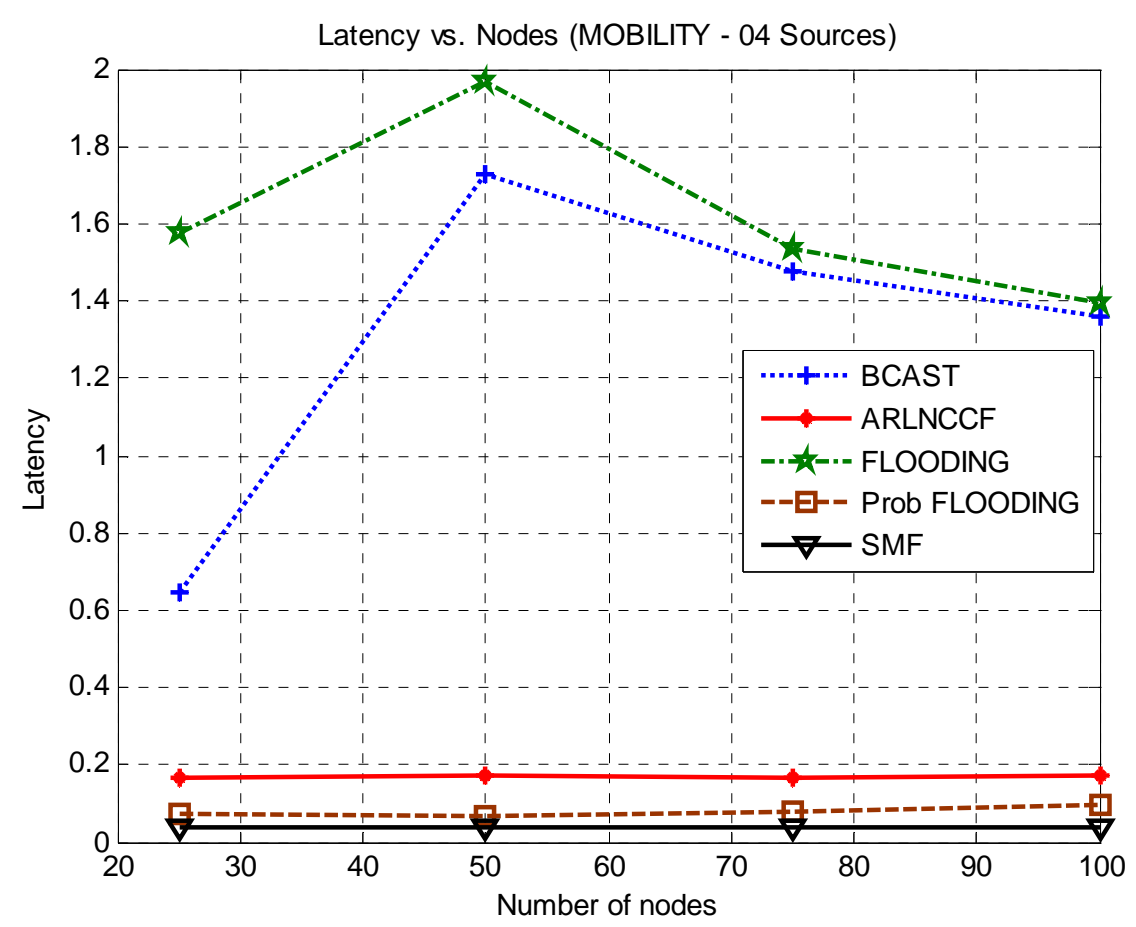

Figure 6.30: Latency vs. Nodes - (Mobility - 04 Sources)

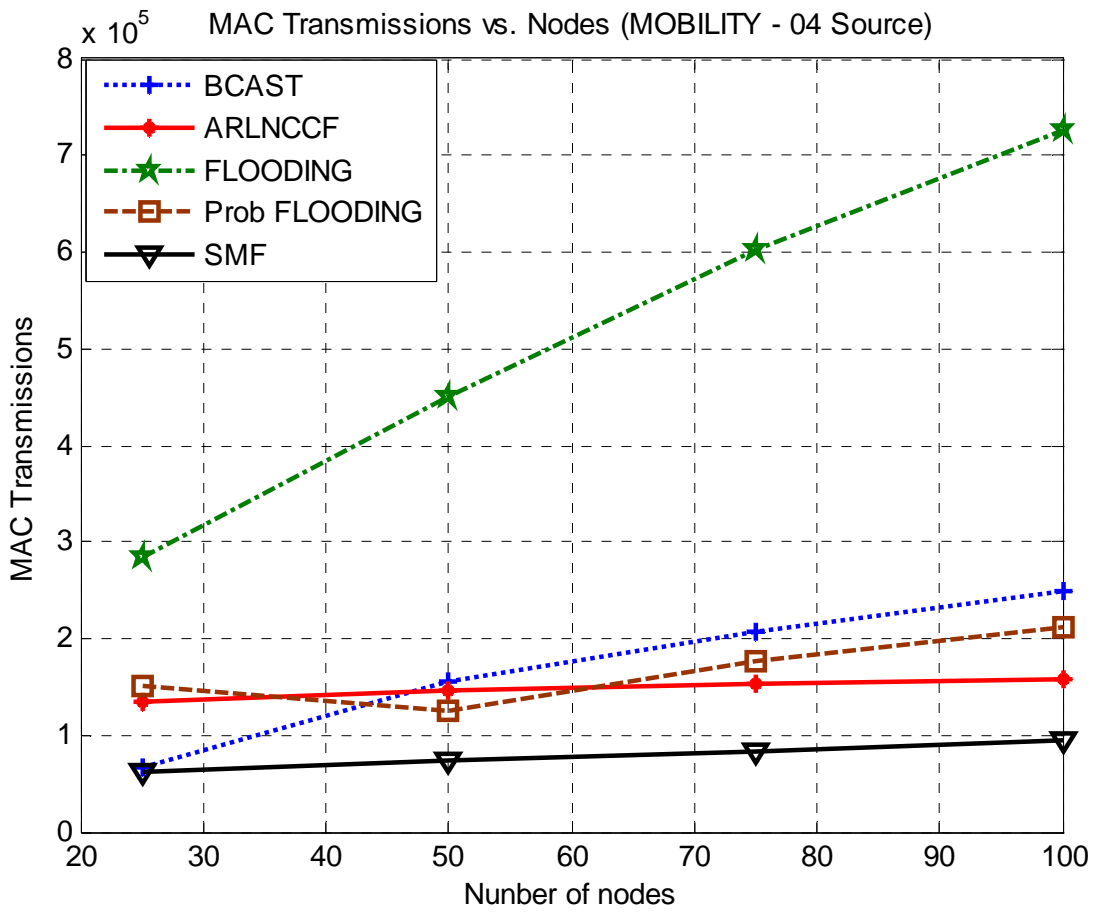

Figure 6.31: MAC Transmissions vs. Nodes - (Mobility - 04 Sources) 


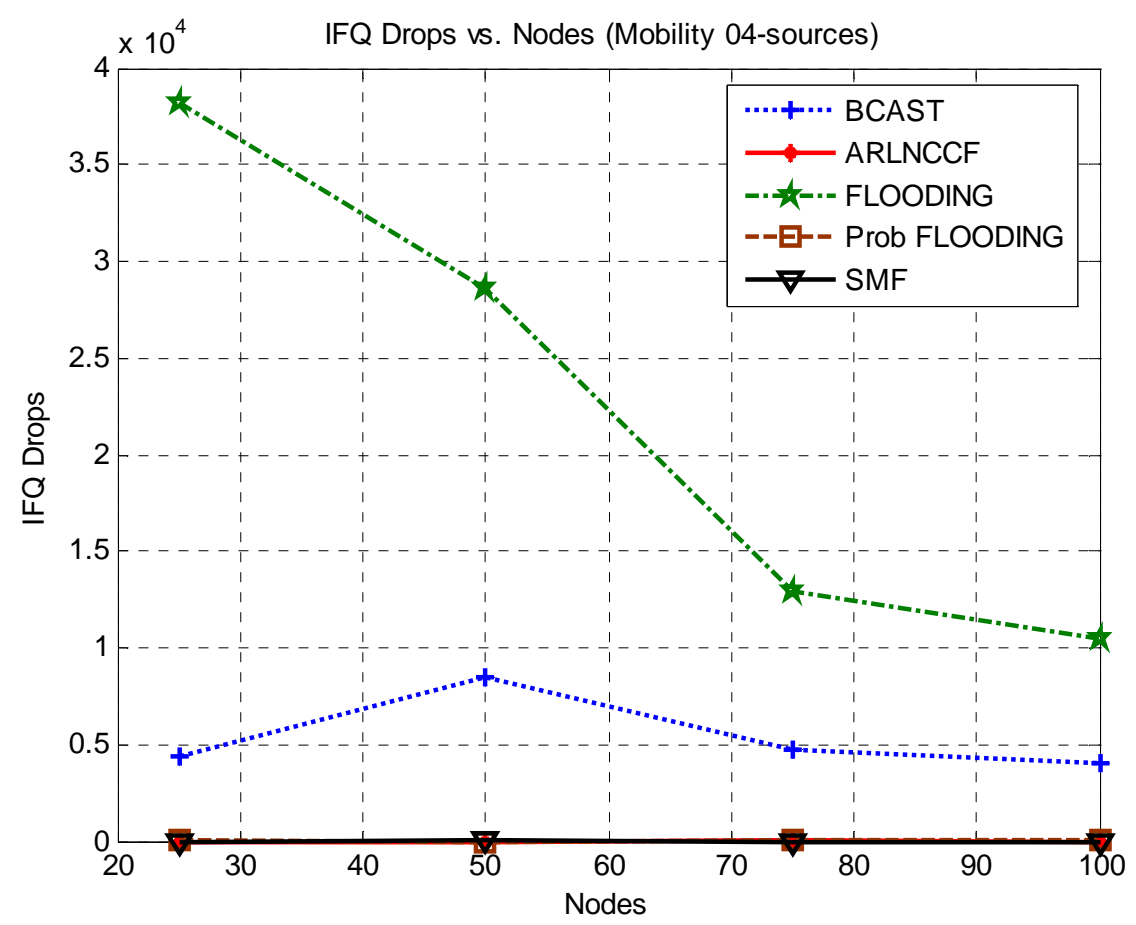

Figure 6.32: IFQ Drops vs. Nodes - (Mobility - 04 Sources)

\subsection{Tactical Scenarios 01-Source}

From the results for static and mobile scenarios, we selected the best candidates, SMF and ARLNCCF to be evaluated for tactical scenarios. Flooding is selected as a baseline protocol for comparison. For the single source case, the PDR for ARLNCCF is slightly better than SMF as shown in Figure 6.33. As the data rate increases from 1.2 kbps to $4.8 \mathrm{kbps}$, the PDR for ARLNCCF shows a slightly decreasing trend. Figure 6.34 shows that the latency for ARLNCCF is higher than SMF due to the generation timeout. The number of MAC transmissions required to achieve this PDR increases with data rate and at $4.8 \mathrm{kbps}$, SMF requires fewer MAC transmissions compared to ARLNCCF as shown in Figure 6.35. 


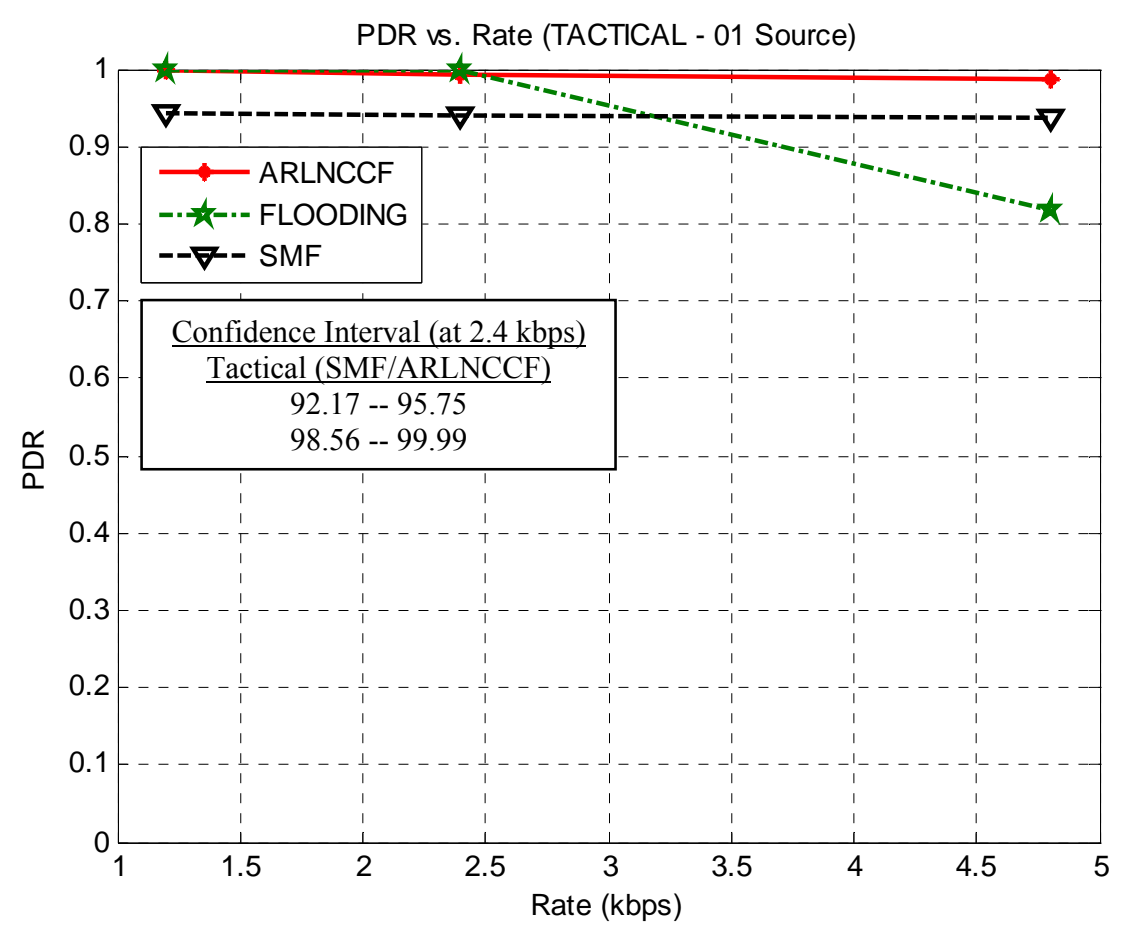

Figure 6.33: PDR vs. Rate (kbps) - (Tactical - 01 Source)

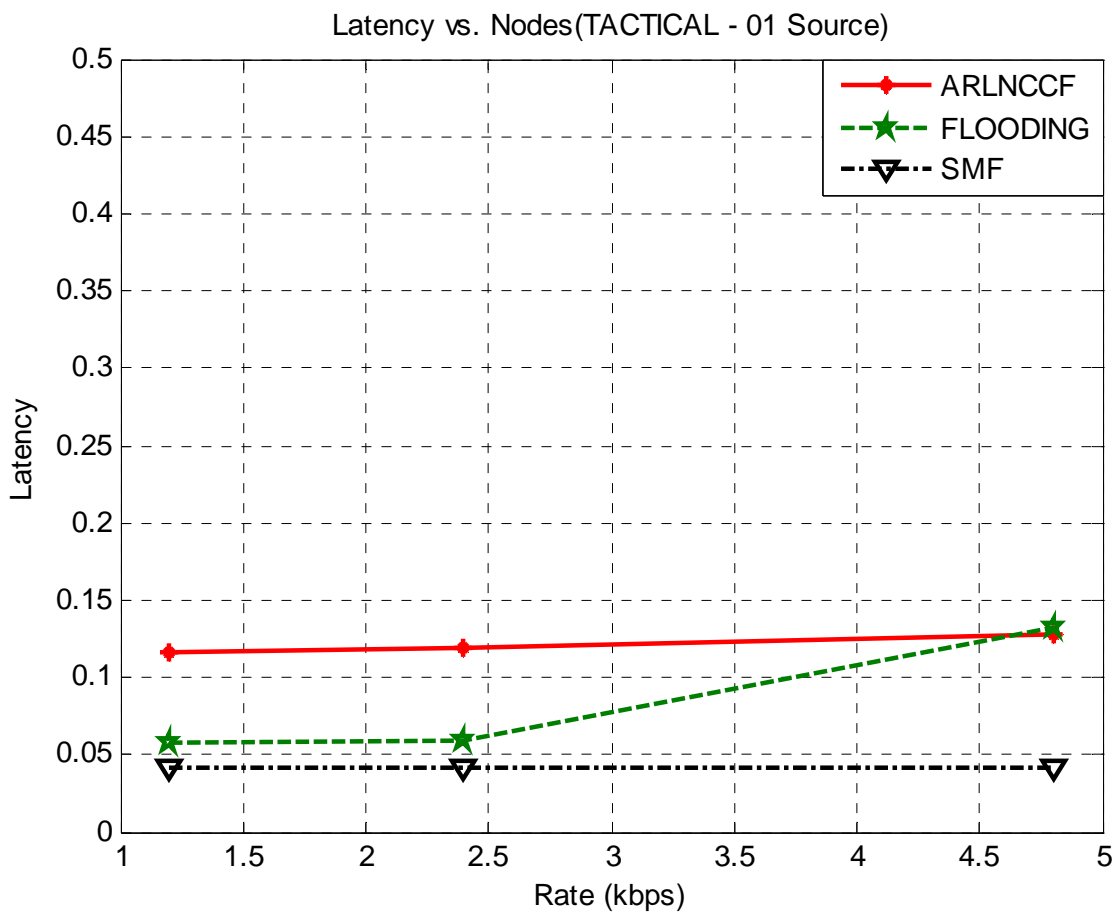

Figure 6.34: Latency vs. Rate (kbps) - (Tactical - 01 Source) 


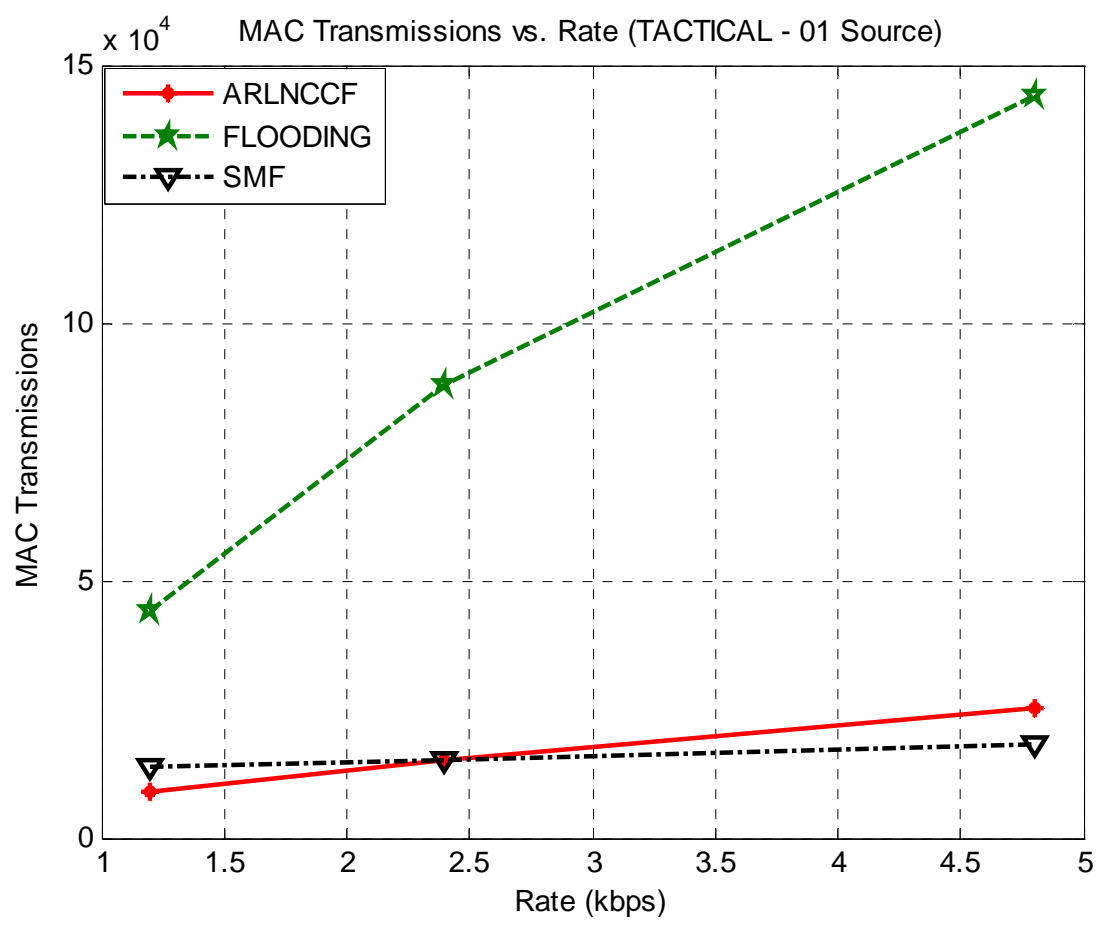

Figure 6.35: MAC Transmissions vs. Rate (kbps) - (Tactical - 01 Source)

\subsection{Tactical Scenarios 04-Sources}

The results for 04-source scenarios show that PDR for ARLNCCF is better than SMF and flooding as shown in Figure 6.36. However, as the data rate increases from 1.2 kbps to $4.8 \mathrm{kbps}$, the PDR for ARLNCCF decreases to around $85 \%$. SMF achieves a PDR of around $83-84 \%$ which remains almost constant as the data rate increases. The obvious difference is in the number of MAC transmissions as shown in Figure 6.38. As the data rate increases, there is an increasing gap in the number of MAC transmissions required to achieve the PDR (Figure 6.36) between ARLNCCF and SMF. SMF shows a slightly poorer PDR than ARLNCCF but achieves this PDR with lower latency and fewer MAC transmissions as shown in Figure 6.37 and 6.38 respectively. 


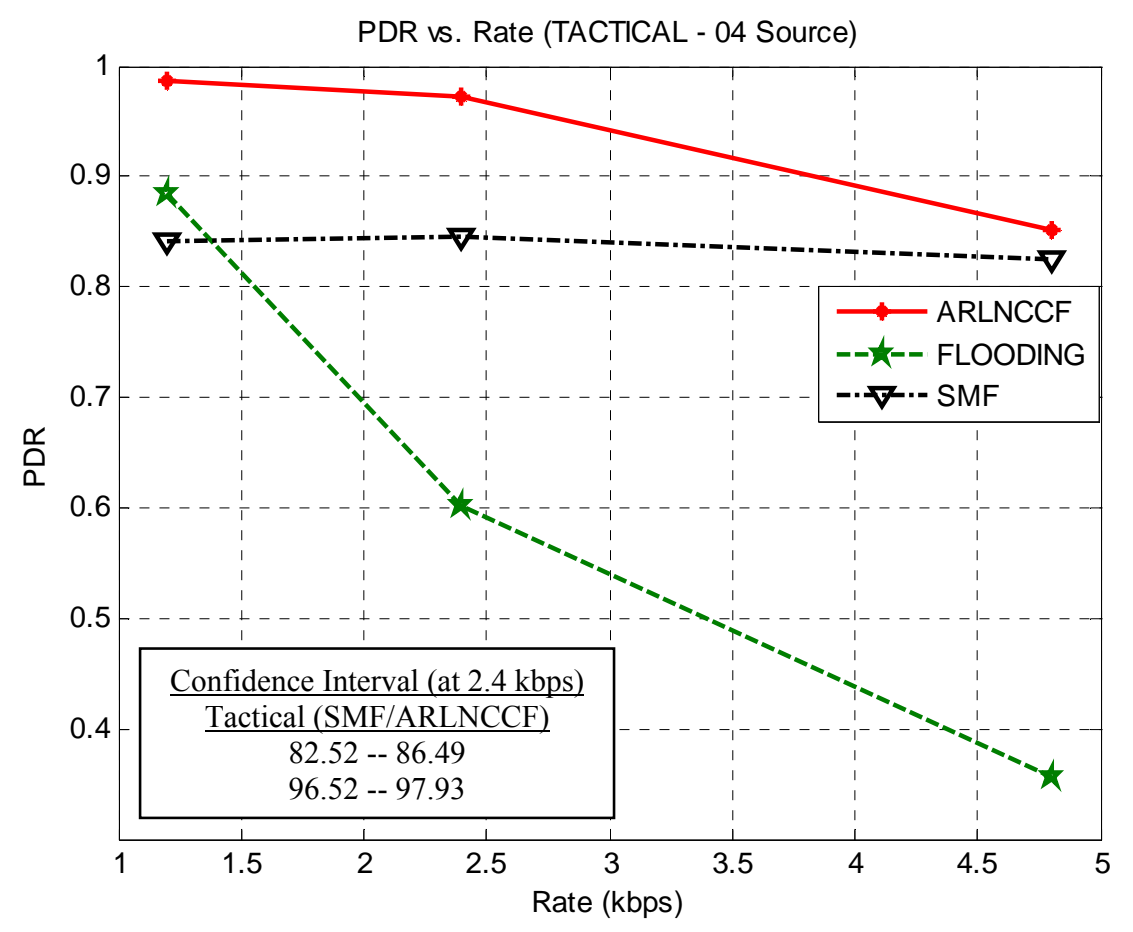

Figure 6.36: PDR vs. Rate (kbps) - (Tactical - 04 Sources)

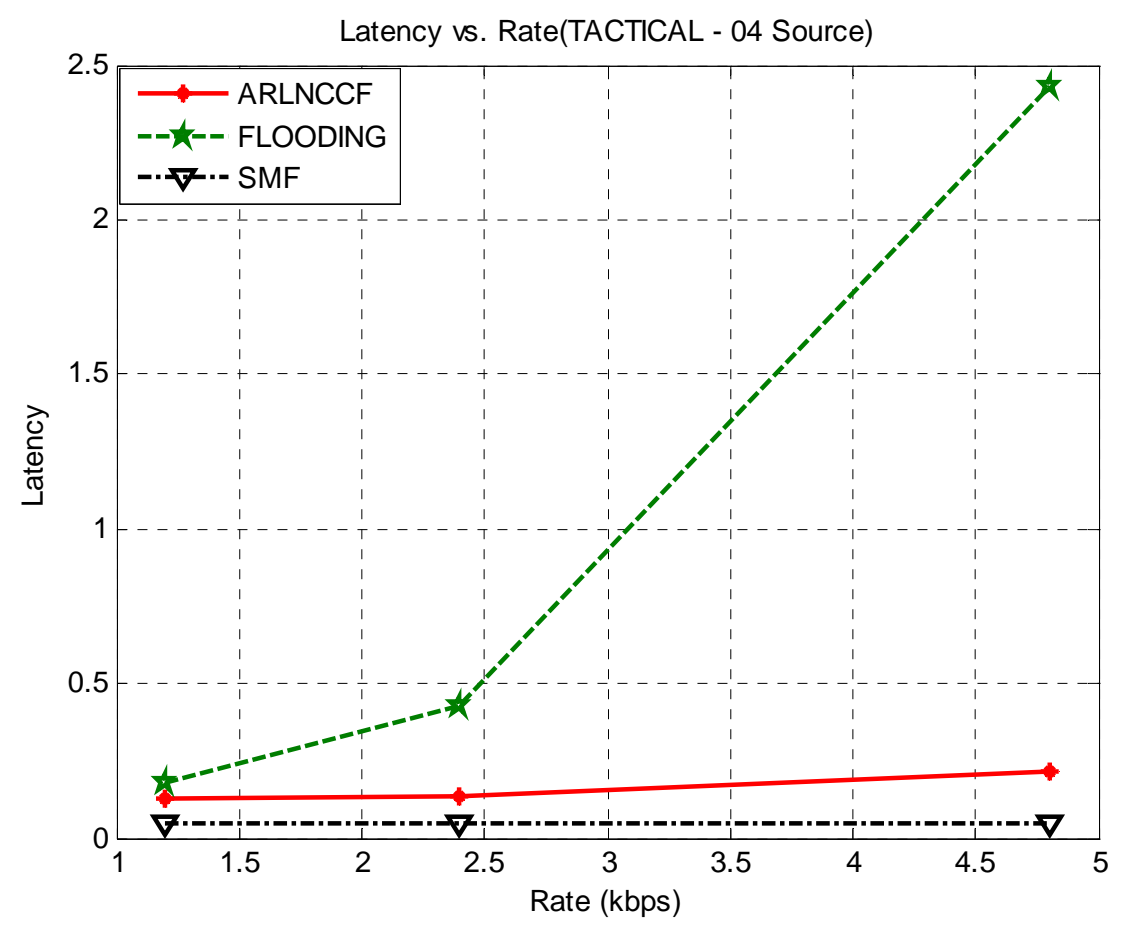

Figure 6.37: Latency vs. Rate (kbps) - (Tactical - 04 Sources) 


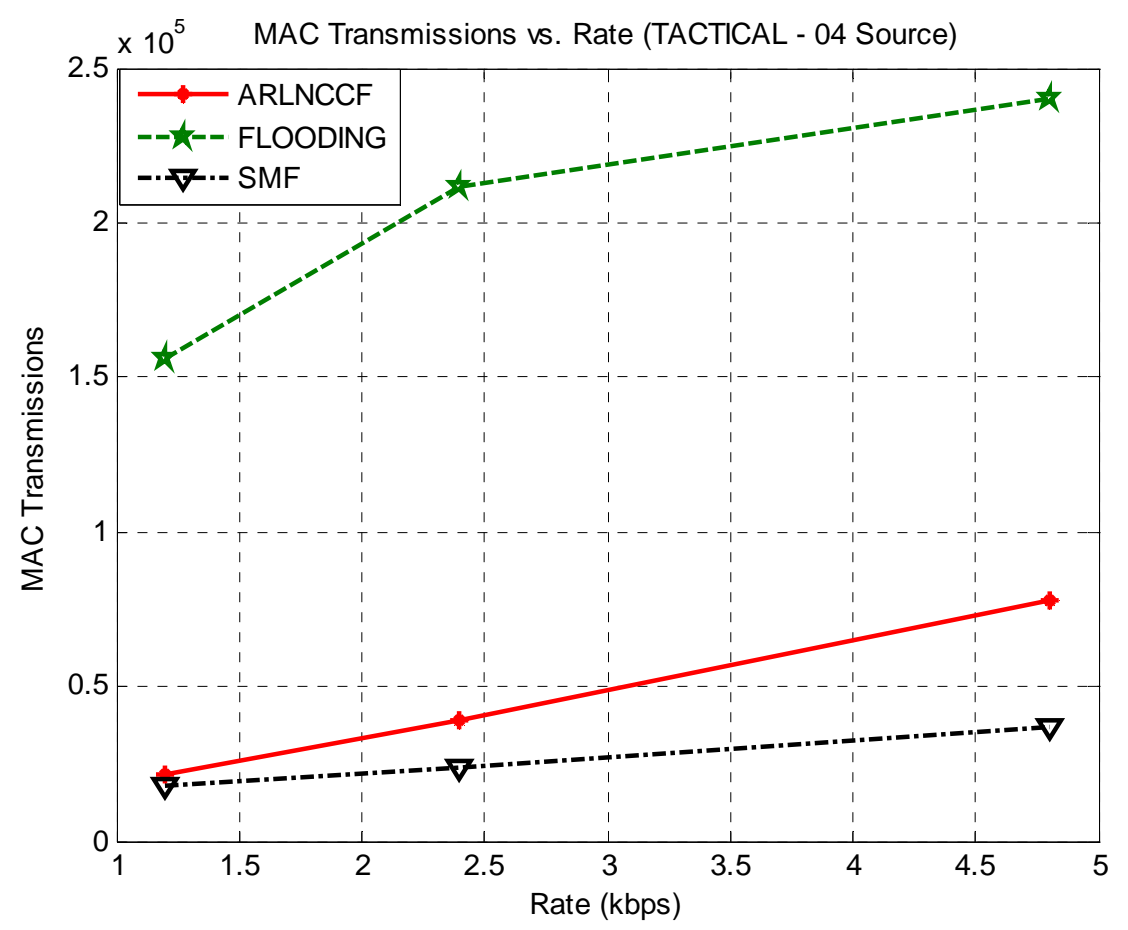

Figure 6.38: MAC Transmissions vs. Rate (kbps) - (Tactical - 04 Sources)

\subsection{Summary}

Overall, ARLNCCF shows a steady performance for different nodes densities and rates for both 01-source and 04-source scenarios. We tested the performance for quite aggressive data rates of up to $100 \mathrm{kbps}$ for 01 -source scenarios and up to $50 \mathrm{kbps}$ for 04 source scenarios. Similarly, the number of nodes is increased from 5 to 100 nodes in the same geographical area. ARLNCCF shows a steady performance in terms of both PDR and latency but there is always some inherent delay due to the need to buffer packets to generate encoding opportunities, which is bounded by the generation timeout. Our protocol, when compared to flooding and BCAST, performs much better. As far as comparison with probabilistic flooding is concerned, since probabilistic flooding is not adaptive to changing scenarios, we need to determine the optimal value of probability of retransmission for each data point considered. Even considering the optimal probability 
value for each scenario, our protocol outperforms probabilistic flooding often. In addition, it adapts well to the changing scenarios.

Now focusing attention to more efficient broadcasting protocols like SMF, we do not see as much benefit of ARLNCCF when compared to SMF. ARLNCCF can be considered as an efficient broadcast protocol, but it cannot be claimed as the best. A direct comparison between SMF and ARLNCCF for Wi-Fi and tactical scenarios is summarized in Table 6.1.

\begin{tabular}{|l|l|}
\hline \multicolumn{1}{|c|}{ Wi-Fi Scenarios } & \multicolumn{1}{|c|}{ Tactical Scenarios } \\
\hline $\begin{array}{l}\text { SMF shows slightly better PDR than } \\
\text { ARLNCCF for static scenarios and } \\
\text { slightly poorer PDR than ARLNCCF for } \\
\text { mobile scenarios, especially for lower data } \\
\text { rates. }\end{array}$ & $\begin{array}{l}\text { ARLenarios. } \\
\text { than SMF in a lossy environment for all }\end{array}$ \\
\hline $\begin{array}{l}\text { Latency for SMF is always lower than } \\
\text { ARLNCCF for all scenarios. }\end{array}$ & $\begin{array}{l}\text { Latency for SMF is always lower than } \\
\text { ARLNCCF for all scenarios. }\end{array}$ \\
\hline $\begin{array}{l}\text { The number of MAC transmissions for } \\
\text { SMF is always lower than ARLNCCF for } \\
\text { all scenarios. }\end{array}$ & $\begin{array}{l}\text { The number of MAC transmissions for } \\
\text { SMF }\end{array}$ \\
\hline
\end{tabular}

Table 6.1: Comparing SMF and ARLNCCF for Wi-Fi and Tactical Scenarios 
We calculated the 95\% confidence intervals between ARLNCCF and SMF for all PDR plots. The 95\% confidence intervals are calculated at $50 \mathrm{kbps}$ (01-source scenarios) and at $25 \mathrm{kbps}$ (04-source scenarios) for varying data rate cases and at number of nodes set to 50 for varying nodes cases for all Wi-Fi scenarios. For tactical scenarios it is calculated at the $2.4 \mathrm{kbps}$ data rate. We verified statistically that the difference in PDR between SMF and ARLNCCF is meaningful at 95\% confidence level. For tactical scenarios this difference is more significant than in Wi-Fi scenarios.

The main points that make SMF better than our protocol in some cases is that the same performance can be achieved at lower latency and fewer MAC transmissions. There are limitations as far as using network coding is concerned. If we compare how our protocol with SMF, we can identify some ways of improving our protocol. SMF uses the concept of relay set selection that causes fewer MAC transmissions. As far as ARLNCCF is concerned, each node contributes towards the transmissions, ensuring that even the least-connected node has a chance to receive enough packets to successfully decode a generation. When multiple nodes are involved in the re-encoding process, the chances that some of the nodes will receive non-innovative packets also increase. If there are more non-innovative transmissions, this causes a higher number of MAC transmissions that are wasted.

If we can apply the concept of relay set selection, it is expected that, once the relay will transmit all the encoded packets in its area and no other node in that area is involved in any transmission, all the transmissions should be innovative for the nodes in that relay set. This causes fewer MAC transmissions as only the relay node is involved in the retransmission and the chances of receiving non-innovative packets also reduces to a 
very large extent. We expect that if the relay set mechanism is applied to ARLNCCF, it will improve the performance of ARLNCCF in terms of fewer MAC transmissions, and may allow it to efficiently support even higher data rates. 


\section{Chapter 7}

\section{Cross-Session Performance}

In this chapter, we investigate the cross-session performance of our protocol. The main aim is to see how much benefit we are getting in allowing inter-mixing of packets from different sources in the same generation. We devised 100-source scenarios as well as 04-source scenarios to investigate the cross-session performance, as described in Chapter 5.

\subsection{0-source Scenarios}

In case of 100-source scenarios, 100 nodes are placed in a $500 \mathrm{~m} * 500 \mathrm{~m}$ area. Using the concept of GD threshold $(\mathrm{GD}=1)$, we are able to control the generation size growth. We observed that the generation size increased from 4 to 8 on average for some generations we monitored closely. Without GD threshold, the generation size becomes

quite large in a dense network of 100 nodes where each node is a source. We observed generation size growth up to 14 or more on average for some monitored generations if we do not use the GD threshold concept. These scenarios are further classified into two types.

1. Each source generates only a single packet during the course of simulation. In the absence of cross-session generations, after the timeout period, since the node generates only one packet, network coding plays no role as there is only 
a single encoded packet. The protocol in this case deteriorates to a simple forwarding approach without coding.

2. Each source generates 4 packets one after another so that at least the generations are full before a timeout occurs, even in the case when we do not allow for cross-session coding.

Table 7.1 shows the PDR performance for both mobile and static scenarios. It is observed that for each case the PDR is better with cross-session generations. The results are further verified via $95 \%$ confidence intervals for each data point. We verified through statistical analysis that the difference is statistically significant and not just due to randomness of simulations.

\begin{tabular}{|c|c|c|}
\hline \multirow{2}{*}{ Scenario } & \multicolumn{2}{|c|}{ PDR (\%) } \\
\hline & No Cross-session & Cross-session \\
\hline $\begin{array}{c}\text { Mobile } \\
(1 \text { packet per source })\end{array}$ & 996.38 & 99.53 \\
\hline Mobile & $87.97 .0145]$ & {$[99.3257-99.7363]$} \\
$(4$ packets per source $)$ & {$[86.2704-89.6716]$} & {$[95.6979-97.2315]$} \\
\hline Static & 95.60 & 99.05 \\
$(1$ packet per source $)$ & {$[94.8930-96.3237]$} & {$[98.4170-99.6970]$} \\
\hline $\begin{array}{c}\text { Static } \\
(4 \text { packets per source })\end{array}$ & $880.1976-84.9664]$ & {$[90.9638-95.0742]$} \\
\hline
\end{tabular}

Table 7.1: PDR for 1 Packet and 4 Packets per Source

Table 7.2 shows the latency performance for both mobile and static scenarios. There is a significant impact on latency when we allow generations to mix packets from different sources (cross-session). For mobile scenarios with 1 packet per source, without cross-session coding, the latency is 0.49 second. Whereas, allowing cross-session coding, the latency is reduced to 0.15 second. In case we allow each source to generate 4 packets, 
without cross-session coding, the latency is 0.32 second compared to 0.185 second for cross-session coding. Similar observations are made for static scenarios. Again, for each value, the $95 \%$ confidence interval is provided to verify our results statistically.

\begin{tabular}{|c|c|c|}
\hline \multirow{2}{*}{ Scenario } & \multicolumn{2}{|c|}{ Latency $(\mathrm{ms})$} \\
\cline { 2 - 3 } & No Cross-session & Cross-session \\
\hline Mobile & 0.49 & 0.155 \\
(1 packet per source) & {$[0.4842-0.5058]$} & {$[0.1459-0.1641]$} \\
\hline Mobile & 0.32 & 0.185 \\
(4 packets per source) & {$[0.3163-0.3317]$} & {$[0.1737-0.1963]$} \\
\hline Static & 0.624 & 0.22 \\
$(1$ packet per source $)$ & {$[0.6058-0.6422]$} & {$[0.2154-0.2371]$} \\
\hline Static & 0.457 & 0.30 \\
$(4$ packets per source $)$ & {$[0.4363-0.4792]$} & {$[0.2785-0.3235]$} \\
\hline
\end{tabular}

Table 7.2: Latency for 1 Packet and 4 Packets per Source

\subsection{4-Source Scenarios}

Figures 7.1 to 7.4 show the cross-session performance of ARLNCCF for 4-source scenarios. Each figure compares the cases with and without cross-session coding for static and mobile scenarios. Figure 7.1 and Figure 7.2 compare the PDR and latency as a function of the data rate and Figure 7.3 and Figure 7.4 compare the PDR and latency as a function of the number of nodes. The results show that, for all cases, the cross-session coding cases have always better PDR as well as lower latency compared to no crosssession coding. As seen in Figure 7.2, as the data rate increases, the latency for no crosssession coding increases significantly for both static and mobile scenarios. The 95\% confidence interval is calculated for a scenario with a date rate of $25 \mathrm{kbps}$ for scenarios where we vary the date rate and for the scenario with the number of nodes set to 50 where we varied number of nodes. 


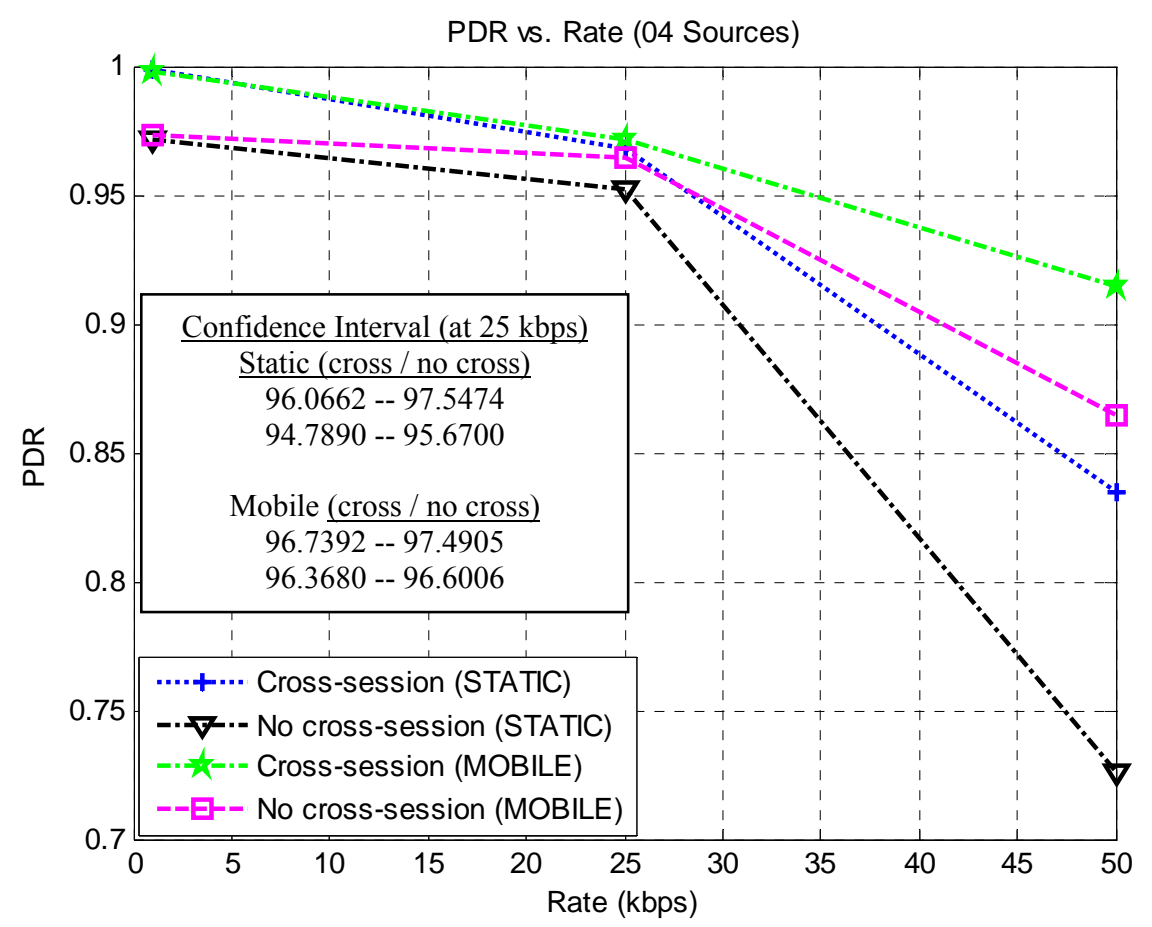

Figure 7.1: PDR vs. Rate (kbps) - (04 Sources)

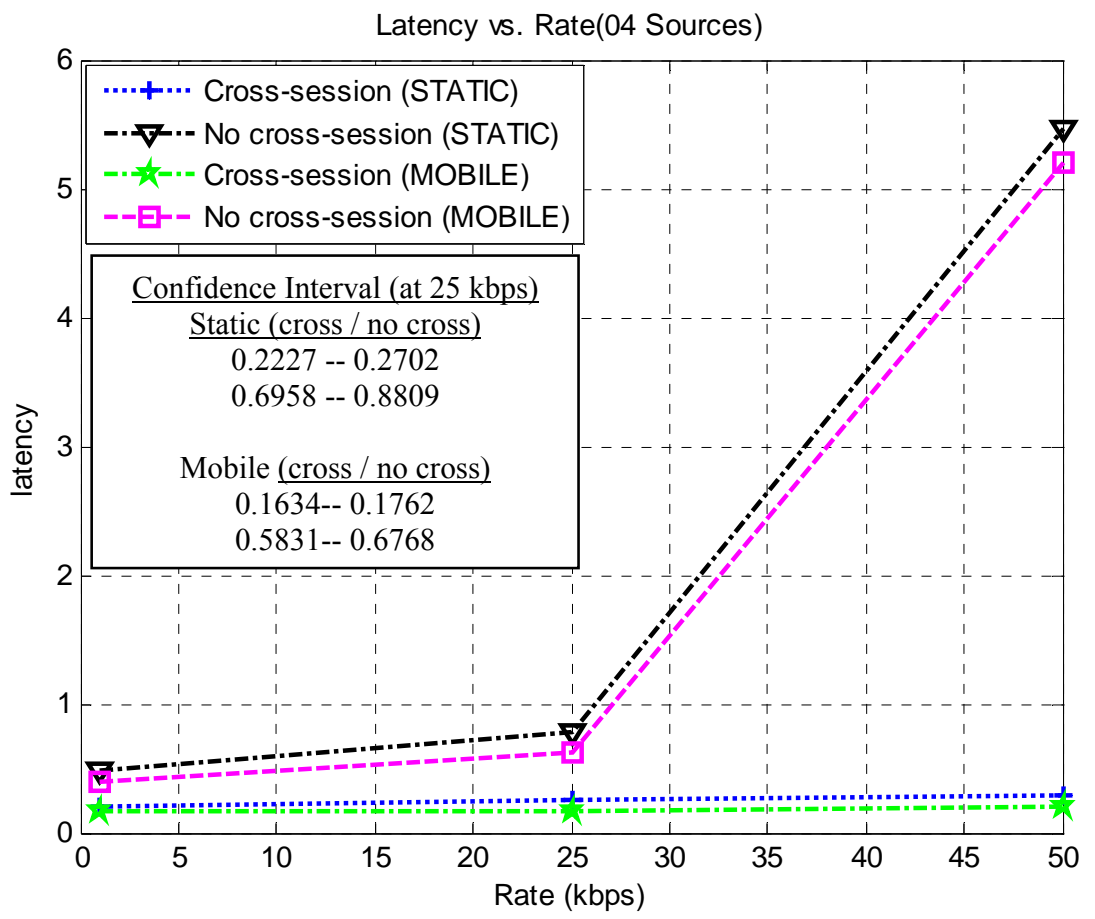

Figure 7.2: Latency vs. Rate (kbps) - (04 Sources) 


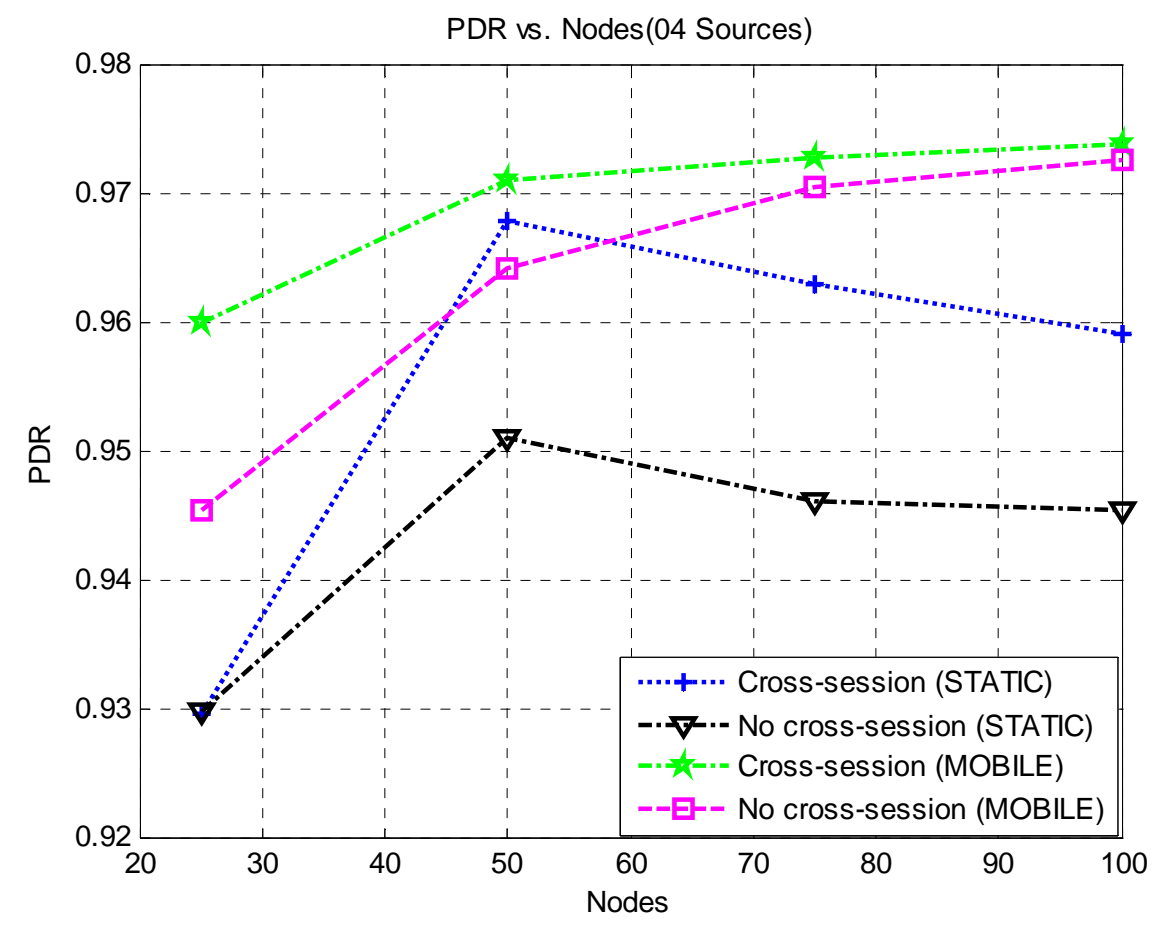

Figure 7.3: PDR vs. Nodes - (04 Sources)

The confidence interval for Figure 7.3 is given in Table 7.3 for the scenarios with 50 nodes.

\begin{tabular}{|c|}
\hline Confidence Interval (at Nodes 50) \\
$\frac{\text { Static }(\text { cross / no cross) }}{96.0662-97.5474}$ \\
$94.6461-95.5877$ \\
Mobile (cross / no cross) \\
$96.7392-97.4905$ \\
$96.2402-96.6179$ \\
\hline
\end{tabular}

Table 7.3: Confidence Interval-PDR (04 Sources - 50 Nodes) 


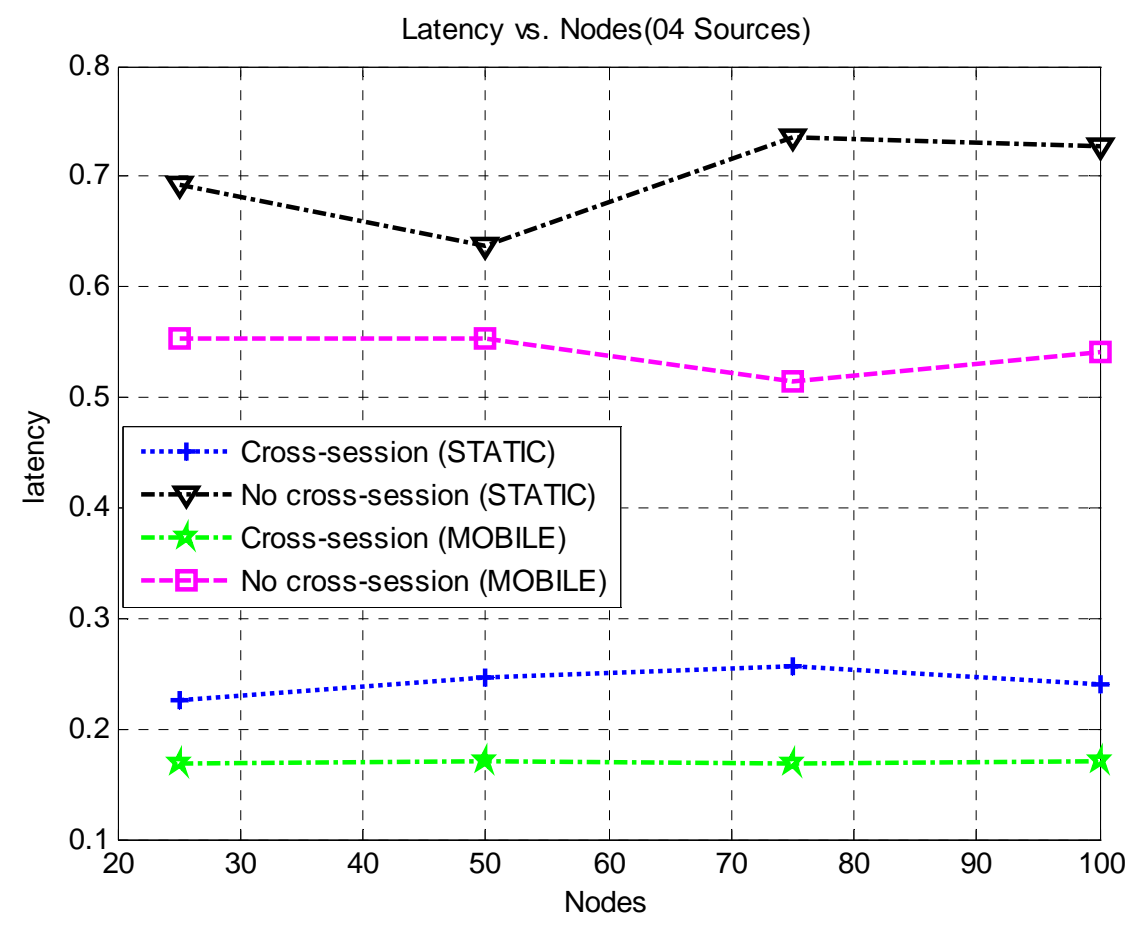

Figure 7.4: Latency vs. Nodes (04 Sources)

The confidence interval for Figure 7.4 is given in Table 7.4 below at number of nodes set to 50 .

\begin{tabular}{|c|}
\hline Confidence Interval (at Nodes 50) \\
\hline Static (cross / no cross) \\
$0.2227-0.2702$ \\
$0.5792-0.6952$ \\
Mobile (cross / no cross) \\
$0.1634-0.1762$ \\
$0.4749-0.6333$ \\
\hline
\end{tabular}

Table 7.4: Confidence Interval-Latency (04 Sources - 50 Nodes) 
In a nutshell, the simulation results show that allowing cross-session coding has a significant impact on PDR as well as latency, which is also statistically verified through $95 \%$ confidence intervals. The concept of allowing cross-session generations in our protocol plays an important role in multi-source scenarios. The results are more profound for mobile scenarios. 


\section{Chapter 8}

\section{Conclusions and Future Work}

\subsection{Conclusions}

In this thesis, we developed a wireless broadcast protocol based on Random Linear Network Coding (RLNC). The following conclusions are drawn as a result of this research work.

- Although there are many broadcast protocols developed so far, very few protocols deal with multi-source RLNC based broadcast. Similarly, none of the RLNC-based protocols are adaptive by design. They need parameters to control the number of required transmissions. Probability-based methods require the optimal values of probability of retransmission set beforehand to achieve better performance in different scenarios.

- Using neighbour knowledge and the generation size, our protocol can effectively calculate the number of rebroadcasts that are sufficient for all the nodes to decode the coded packets. We tested our protocol in static, mobile and tactical scenarios for both 01-source and 04-source cases. Similarly, we investigated the performance by varying the number of nodes as well as data rates in these scenarios. Our simulation results demonstrate the potential of our algorithm to support wireless broadcast in adhoc networks. We observe a steady performance of our protocol at different node densities and data rates in all scenarios, thus making our protocol adaptive. 
- During the simulations, we tested our protocol for very aggressive data rates (not used in the literature before for RLNC based broadcast). As an example [9] used $40 \mathrm{kbps}$ and [15] used $50 \mathrm{kbps}$ for single source scenarios. It is mentioned in [15] that their RLNC based probabilistic broadcast algorithm has high packet loss for higher data rates. We used up to $100 \mathrm{kbps}$ for a single source and up to $25 \mathrm{kbps}$ per source for 04source scenarios. It was observed that the PDR performance was not degraded. This shows that by controlling the number of rebroadcasts, we are in fact able to achieve better performance at higher data rates.

- When we compare our results with SMF, we observe that SMF achieves the same or better PDR by using fewer MAC transmissions than our protocol in certain cases. Similarly, our protocol has an inherent delay due to the generation timeout, as a result latency is higher than SMF, but it does not increase with increasing node densities or data rates and remains consistent.

- By using the concept of earlier decoding from previous research, we are able to decode some of the original packets before the generation is complete. This greatly reduces the decoding delays.

- Since our protocol is designed for multi-source environments and we allow packets from different sources to be combined in the same generation (cross-session) whenever possible, there is always a possibility that the generation size can grow to a very large extent, especially for high data rates and multi-hop networks. This is causing decoding complexity, decoding delays, and very large packet sizes. In order to avoid this situation, we introduced the concept of Generation Distance (GD). GD values can be set to control the source nodes entering their packets in the given 
generation based on the distance (hops) from the generation origin. However as future work we need to investigate further the impact of GD as well as devising more efficient ways to control generation size.

- We have shown that our concept of cross-session generations plays an important role in improving the PDR as well as reducing latency. We specifically designed 100source scenarios to test the cross-session concept. Similarly, we used the original 04source scenarios by varying the number of nodes and data rate. We demonstrated through these simulations that for every scenario, cross-session coding results in higher PDR and lower latency compared to no cross-session coding, where coding opportunities are limited to packets originating from the same source. The difference in performance is statistically significant at a $95 \%$ confidence level.

\subsection{Future Work}

During the work on this thesis, possibilities of future work have arisen. They are summarized as following:

1. By comparing the performance of ARLNCCF and SMF, a very promising idea is to combine the concept of a Relay Set used in SMF with ARLNCCF. The new protocol will require a new equation to calculate the number of retransmissions required based on the Relay Sets. It is expected that the new protocol will require fewer MAC transmissions than ARLNCCF and will improve the PDR performance as well. This idea is already discussed in some more detail in Section 6.11.

2. Although our protocol is adaptive to network density, some of the parameters are still fixed, such as generation size and generation timeout. As a future work, some scheme 
can be developed that will adapt the generation size and timeout values to changing scenarios. The timeout value is dependent upon the generation size and also the data rate. The generation size is dependent upon the number of sources and node density. In a dense network environment with more sources, choosing a large generation size is not recommended, as the generations will potentially grow beyond that value. However, for example for single source scenarios, we can afford bigger generation sizes.

3. In our packet format, we have used the IP address and sequence number pair to identify the packets for a particular source. More efficient mechanism can be devised to identify the packets for particular sources, which can reduce the packet size and as a result reduce the overhead. As an example, some hashing mechanism can be devised to identify the packets per source.

4. Using our protocol approach, analytical analysis can be done by selecting some simple topologies and investigating the performance of our protocol. This will provide a solid base for our approach.

5. In our current simulations, we have assumed unlimited buffer space available in the nodes. However, in real scenarios, the nodes have limited memory. In the future work, the memory consideration can also be included in the protocol design. Based on the available buffer space, a generation management scheme needs to be devised. A new approach can be developed which could work well for generation management keeping in mind the generation parameters. 


\section{References}

1. S. Ni, Y. Tseng, Y. Chen, and J. Sheu, "The Broadcast Storm Problem in a Mobile Ad Hoc Network” IEEE/ACM Mobile Computing and Networking, pages $151-162,1999$.

2. C. Fragouli, D. Katabi, A. Markopoulou, M. Médard, and H. Rahul, “Wireless Network Coding: Opportunities and Challenges", IEEE Military Communications Conference, pages 1-7, Oct. 2007.

3. R. Ahlswede, N. Cai, S. R. Li, and R. W. Yeung, "Network Information Flow," IEEE Transactions on Information Theory, pages 1204-1216, Jul. 2000.

4. R. Khalili, M. Ghaderi, J. Kurose and D. Towsley, "On the Performance of Random Linear Network Coding in Relay Networks", IEEE Military Communication, pages 1-7, Nov 2008.

5. D. S. Lun, M. Médard, and R. Koetter, "Efficient Operation of Wireless Packet Networks using Network Coding”, International Workshop on Convergent Technologies (IWCT), Invited paper, pages 1-5, Jun. 2005.

6. E. Ahmed, A. Eryilmaz, M. Médard, and A. E. Ozdaglar, "On the Scaling Law of Network Coding Gains in Wireless Networks”, IEEE Military Communications Conference, Oct. 2007.

7. M. Wang and B. Li, "Lava: A Reality Check of Network Coding in Peer-to-Peer Live Streaming”, IEEE International Conference on Computer Communications, pages 1082-1090, May 2007. 
8. C. Fragouli, J. Y. Boudec, and J. Widmer, "Network Coding: An Instant Primer”, ACM Special Interest Group on Data Communication, Computer Communication Review, pages 63-68, Vol. 36, No. 1, Jan. 2006.

9. J. S. Park, D. Lun, Y. Yi, M. Gerla, and M. Medard, "Codecast: A Network Coding Based Adhoc Multicast Protocol”, IEEE Wireless Communications, pages 76-81, Oct. 2006.

10. M . Ghaderi, D. Towsley, J. Kurose, "Reliability Gain of Network Coding in Lossy Wireless Networks", IEEE International Conference on Computer Communications, pages 1-23, Mar. 2008.

11. C. Gkantsidis and P. Rodriguez, "Network Coding for Large Scale Content Distribution”, IEEE International Conference on Computer Communications, pages 1-11, 2005.

12. N. Jayakumar, K. Gulati, S. Khatri, and A. Sprintson, "Network Coding for Routability Improvement in VLSI", IEEE/ACM International Conference on Computer-Aided Design, pages 820-823, Nov. 2006.

13. A. G. Dimakis, P. B. Godfrey, M. Wainwright, and K. Ramchandran, "Network Coding for Distributed Storage Systems", IEEE Conference on Computer Communications, pages 1-12, May 2007.

14. J. Widmer, J. Boudec, "Network Coding for Efficient Communication in Extreme Networks", ACM Special Interest Group on Data Communication, Pages $284-291,2005$. 
15. S. Tarapiah, C. Casetti, C. Chiasserini, "Network Coding in Ad Hoc Networks: A Realistic Simulation Study", IEEE International Conference on Computer Communications, pages 1-2, Apr. 2009.

16. C. Fragouli, J. Widmer, and J. Y. L. Boudec, "Efficient Broadcasting Using Network Coding", IEEE/ACM Transactions on Networking, pages 450-453, 2008.

17. T. Ho, M. Médard, R. Koetter, D. R. Karger, M. Effros, J. Shi, and B. Leong, “A Random Linear Network Coding Approach to Multicast”, IEEE Transactions on Information Theory, vol. 52, pages 4413-4430, Oct. 2006.

18. K. Mahmood, T. Kunz, and A. Matrawy, "Adaptive random linear network coding with controlled forwarding for wireless broadcast", Proceedings of the IFIP Wireless Days 2010, Venice, Italy, Oct. 2010

19. Y. Wu, "Network Coding for Wireless Networks", Microsoft Research Technical Report, MSR-TR-2007-90, pages 1-27, Jul. 2007.

20. S. Katti, H. Rahul, W. Hu, D. Katabi, M. Médard and J. Crowcroft, "XORs in the Air: Practical Wireless Network Coding”, IEEE/ACM Transactions on Networking, pages 497-510, Jun. 2008.

21. V. Bhargava and S. Wicker, "Reed-Solomon Codes and Their Applications", IEEE Press, ISBN 0-7803-5391-9, 1994.

22. L. Li, R. Ramjee, M. Buddhikot, and S. Miller, "Network-coding based Broadcast in Mobile Ad-hoc networks", IEEE Conference on Computer Communications, pages 1739-1747, Apr. 2007. 
23. S. R. Li, R. W. Yeung, and N. Cai, “Linear Network Coding”, IEEE Transactions on Information Theory, pages 371-381, Feb. 2003.

24. C. Fragouli, J. Widmer, and J.-Y. L. Boudec, “A Network Coding Approach to Energy Efficient Broadcasting: From Theory to Practice”, IEEE Conference on Computer Communications, pages 1-11, Apr. 2006.

25. A. Argyriou, R. Philips, “Wireless Network Coding with Improved Opportunistic Listening”, IEEE Transactions on Wireless Communications, pages 2014-2023, April 2009.

26. R. Khalili, M. Ghaderi, J. Kurose, D. Towsley, “On the Performance of Random Linear Network Coding in Relay Networks", IEEE Military Communications Conference, pages 1-7, Nov. 2008.

27. N. Cai and R. W. Yeung, "Secure Network Coding", International Symposium on Information Theory, page 323, 2002.

28. B. Williams and T. Camp, "Comparison of Broadcasting Techniques for Mobile Ad hoc Networks", ACM International Symposium on Mobile Ad Hoc Networking and Computing, pages 194-205, 2002.

29. A.R. Chowdhury, S. Nandi, "Survey of Broadcasting Techniques for Dense Wireless Computing Devices", Wireless and Optical Communications Networks, page 9, 2006.

30. T. Kunz, “Multicast Versus Broadcast in a Manet” Lecture Notes in Computer Science, vol. 3158. Springer: Berlin, pages 14-27, 2004.

31. "Optimized Link State Routing Protocol (OLSR)", Request For Comment \# 3626 “http://www.ietf.org/rfc/rfc3626.txt”, Oct. 2003. 
32. "Topology Dissemination Based on Reverse-Path Forwarding (TBRPF)", Request For Comment \# 3684, “http://www.ietf.org/rfc/rfc3684.txt”, Feb. 2004.

33. "Simplified Multicast Forwarding (SMF)", IETF Draft, “http://tools.ietf.org/html/draft-ietf-manet-smf-09”, Jul. 2009.

34. H. Lim and C. Kim, “Flooding in Wireless Ad hoc Networks", Computer Communications Journal, volume 24, pages 353-363, 2001.

35. W. Lou and J. Wu, “On Reducing Broadcast Redundancy in Ad hoc Wireless Networks", IEEE Transactions on Mobile Computing, pages 111 - 122, AprilJun. 2002.

36. R. Koetter and M. Médard, “An Algebraic Approach to Network Coding”, IEEE/ACM Transactions on Networking, pages 782-795, 2003.

37. D. S. Lun, M. Médard, and R. Koetter. "Efficient Operation of Wireless Packet Networks using Network Coding”, International Workshop on Convergent Technologies, pages 1-5, Jun 2005.

38. Y. Wu, P. A. Chou, and K. Jain, “A Comparison of Network Coding and Tree Packing”, IEEE International Symposium on Information Theory, 2004.

39. M. Ghaderi, D. Towsley, J. Kurose, “Network Coding Performance for Reliable Multicast”, IEEE Military Communications Conference, pages 1-7, Oct. 2007.

40. B. Radunovic, C. Gkantsidis, P. Key, S. Gheorgiu, W. Hu, and P. Rodriguez, “Multipath Code Casting for Wireless Mesh Networks”, ACM International Conference on Emerging Networking Experiments and Technologies, pages 1-16, 2007. 
41. X. Zhang and B. Li, “Optimized Multipath Network Coding in Lossy Wireless Networks”, IEEE International Conference on Distributed Computing Systems, pages 243-250, 2008.

42. T. Matsuda, T. Noguchi, T. Takine, “Broadcasting with Randomized Network Coding in Dense Wireless Ad Hoc Networks", IEICE Transactions on Communications, pages 3216-3225, 2008.

43. Y. Kondo, H. Yomo, S. Yamaguchi, P. Davis, R. Miura, S. Obana, “Reliable Wireless Broadcast with Random Network Coding for Real-Time Applications”, IEEE Conference on Wireless Communications and Networking, Pages 1-6, 2009.

44. P. A. Chou, Y. Wu, and K. Jain, “Practical Network Coding” $41^{\text {st }}$ Annual Allerton Conference on Communication, Control, and Computing, pages 1-10, Oct. 2003.

45. W. H. Press, “Numerical Recipes: The Art of Scientific Computing”, ISBN-10: 0521880688, Third Edition, Cambridge University Press 2007.

46. T. Kunz, “Efficiently Supporting One-to-Many and Many-to-Many Communication Patterns in Narrowband Tactical Networks: Flooding, Efficient Broadcasting, and Network Coding”, Technical Report, Mar. 2009

47. R. Punnoose, P. Nikitin, and D. Stancil, “Efficient Simulation of Ricean Fading within a Packet Simulator”, IEEE Vehicular Technology Conference, pages 764$767,2000$.

48. N. R. Wagner, "The Laws of Cryptography with Java Code", online at Neal Wagner's home page, http://www.cs.utsa.edu/ wagner/ 
49. P. Vingelmann, "Network Coding on the GPU”, Master's Thesis, Budapest University of Technology, 2009. 


\section{Appendix A}

\section{Encoding Process:}

The encoder is responsible to create coded messages from the original packets. $K$ consecutive bits of a packet can be divided into $L=K / s$ symbols, where a symbol has s bits as shown in Figure A.1.

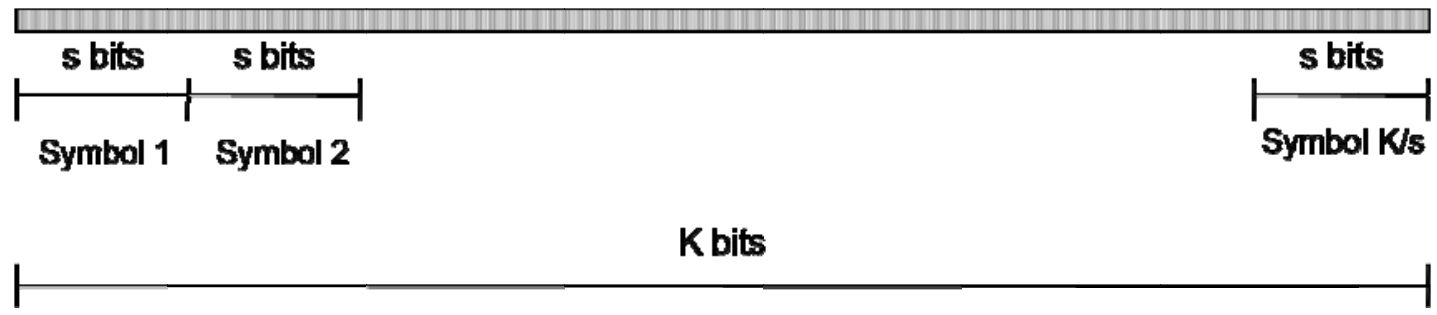

Figure A.1: Encoding Process

These symbols can be interpreted as taken from the Galois Field $G F\left(2^{\mathrm{s}}\right)$, with each packet consisting of a vector of $K / s$ symbols. A Galois Field has a finite number of elements and all the arithmetic operations in GF also result in the same finite field elements. Due to this reason, it is well suited for RLNC. Assume that a number of original packets $B_{1}, B_{2}, \ldots, B_{N}$ are generated by one or several sources. To code a new packet $X_{j}$ of size $L(K / s$ symbols $)$, a node chooses a set of coding coefficients $\boldsymbol{c}_{\boldsymbol{j}}=\left[\boldsymbol{c}_{\boldsymbol{j} 1}, \boldsymbol{c}_{\boldsymbol{j} 2}\right.$, $\left.\ldots, c_{j N}\right]$ in $G F\left(2^{s}\right)$, so there is one coefficient for each original packet [49]. The new coded packet is equal to: $X_{j}=\sum_{i=1}^{N} c_{j i} \times B_{i}$

The summation has to occur for every symbol position. So this expression can be rewritten as a matrix multiplication by organizing the coding coefficients $(C)$ and the 
original packets $(B)$ into matrices. The matrix form of the previous expression is: $X=C$ $\times B$

The weights or coefficients are selected randomly and independently from this GF. That is why the approach is referred to as Random Linear coding, as the transformation is performed by each node independently of others and using random coefficients [8]. The coding coefficients are in the form of a vector called Coding Vector, $c_{j}$. The coding vector is sent in the header of the packet containing the encoded data called information vector $\boldsymbol{X}_{\boldsymbol{j}} \cdot\left(c_{j}, X_{j}\right)$ represents the coded packet $j$.

\section{Arithmetic operation:}

Considering GF $\left(2^{8}\right)$, the required arithmetic operations are implemented in the following way:

- Addition and subtraction are simply XOR operations.

- Multiplication is more complicated. The first step in multiplying two field elements is to multiply their corresponding polynomials just as in algebra (except that addition is via the XOR operation). The result might be a value greater than 1 byte. So the finite field now makes use of a fixed degree eight irreducible polynomial (a polynomial that cannot be factored into the product of two simpler polynomials). Multiplication modulo that irreducible reducing polynomial gives the final result. In case of the Advanced Encryption Standard (AES), the polynomial used is

$$
x^{8}+x^{4}+x^{3}+x+1=0 x 11 b(h e x)
$$




\section{Example:}

For example, $0 \times 93 * 0 \times 51$ can be calculated as follows:

$$
\begin{aligned}
& \left(x^{7}+x^{4}+x+1\right) *\left(x^{6}+x^{4}+1\right) \\
& =x^{13}+x^{11}+x^{7}+x^{10}+x^{8}+x^{4}+x^{7}+x^{5}+x+x^{6}+x^{4}+1 \\
& =x^{13}+x^{11}+x^{10}+x^{8}+x^{6}+x^{5}+x+1 \\
& \text { 0x93*0x51 } \\
& =\left(x^{13}+x^{11}+x^{10}+x^{8}+x^{6}+x^{5}+x+1\right) \%\left(x^{8}+x^{4}+x^{3}+x^{1}+1\right) \\
& =10110101100011 \% 100011011 \\
& =10000001 \\
& =0 x 81
\end{aligned}
$$

\section{Simple method:}

A simple calculation method that is easily implementable in code is given in [48]. Write the operands in terms of power of the polynomial, i.e. $\left(x^{7}+x^{4}+x+1\right)=(741$ 0). Calculations are done working from the low order terms, and by repeatedly multiplying by (1). If the result reaches degree 8, just add (XOR) the irreducible polynomial ( $\left.8 \begin{array}{llll}8 & 3 & 1 & 0\end{array}\right)$ to obtain a lower degree.

\section{Same example:}

$$
r^{*} s=\left(\begin{array}{llll}
7 & 4 & 1 & 0
\end{array}\right) *\left(\begin{array}{lll}
6 & 4 & 0
\end{array}\right)
$$




\begin{tabular}{|c|c|c|c|}
\hline$i$ & powers of $r: r *$ (i) & Simplified Result & Final Sum \\
\hline 0 & & $\left(\begin{array}{llll}7 & 4 & 1 & 0\end{array}\right)$ & $(74$ \\
\hline 1 & $\left(\begin{array}{llll}7 & 4 & 1 & 0\end{array}\right) *(1)=$ & 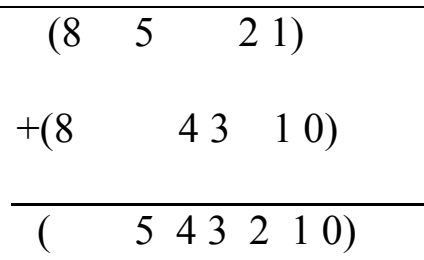 & \\
\hline 2 & $\left(\begin{array}{ll}5432 & 0\end{array}\right) *(1)$ & 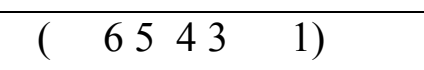 & \\
\hline 3 & $(6543 \quad 1) *(1)$ & $\left(\begin{array}{lll}7654 & 2\end{array}\right)$ & \\
\hline 4 & $(76542) *(1)$ & $\begin{array}{r}\left(\begin{array}{l}8765 \\
8\end{array}\right) \\
+(8 \quad 43 \quad 10)\end{array}$ & 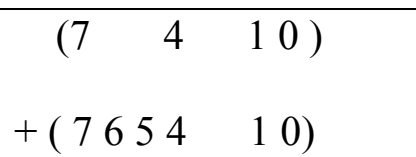 \\
\hline & & $\left(\begin{array}{ll}7654 & 10\end{array}\right)$ & $(65)$ \\
\hline 5 & $\left(\begin{array}{ll}7654 & 10) *(1)\end{array}\right.$ & 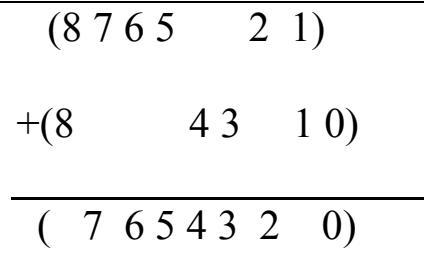 & \\
\hline 6 & $\left(\begin{array}{l}765432 \\
0) *(1)\end{array}\right.$ & 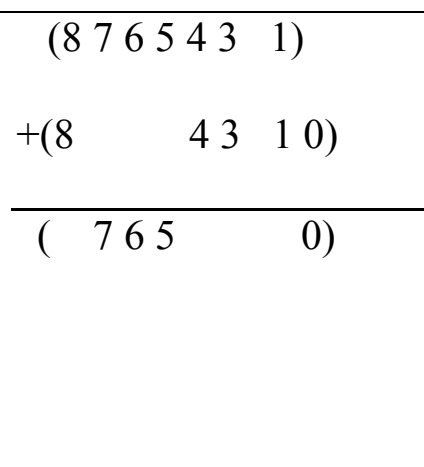 & $\begin{aligned} & \left(\begin{array}{ll}65 \\
6\end{array}\right) \\
+ & \left(\begin{array}{ll}765 & 0\end{array}\right) \\
(7 & 0)= \\
& \\
10000001 & \\
& =0 \times 81\end{aligned}$ \\
\hline
\end{tabular}

Table A.1: Simplified Multiplication on GF $\left(2^{8}\right)$

\section{Example for Encoding in RLNC:}

Suppose we have 4 bytes $(5,6,111,152)$ to be encoded. We select $4 * 4=16$ coding coefficients as 4 coded packets are to be sent for the 4 symbols encoded together. The 
example is provided using coding vector 3 to calculate the second coded symbol in the encoded packet 3 (boxed values). The (randomly selected) coding vectors are as follows:

Coding vector 1: 41, 35, 190, 132

Coding vector 2: 225, 108, 214, 174

Coding vector 3: $82,144,73,241$

Coding vector 4: 241, 187, 233, 235

The encoding is done as follows. Note that the operation here is over $\operatorname{GF}\left(2^{8}\right)$.

1st encoded byte: $41 * 5+35 * 6+190 * 111+132 * 152=209$

2nd encoded byte: $225 * 5+108 * 6+214 * 111+174 * 152=40$

3rd encoded byte: $82 * 5+144 * 6+73 * 111+241 * 152=140$

4th encoded byte: $241 * 5+187 * 6+233 * 111+235 * 152=195$

Now we can form 4 packets, each consisting of a coding vector and the encoded byte, with a size of 5 bytes:

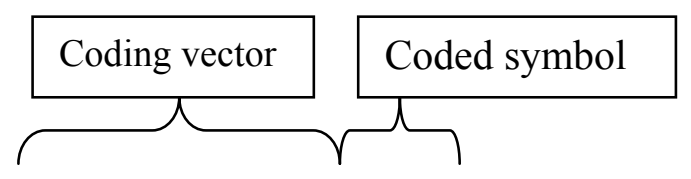

Packet 1: 41, 35, 190, 132, 209

Packet 2: 225, 108, 214, 174, 40

Packet 3: 82, 144, 73, 241, 140

Packet 4: 241, 187, 233, 235, 195 
In the next example, we encode 4 packets (instead of 4 bytes as shown above), each with a size of 10 bytes:

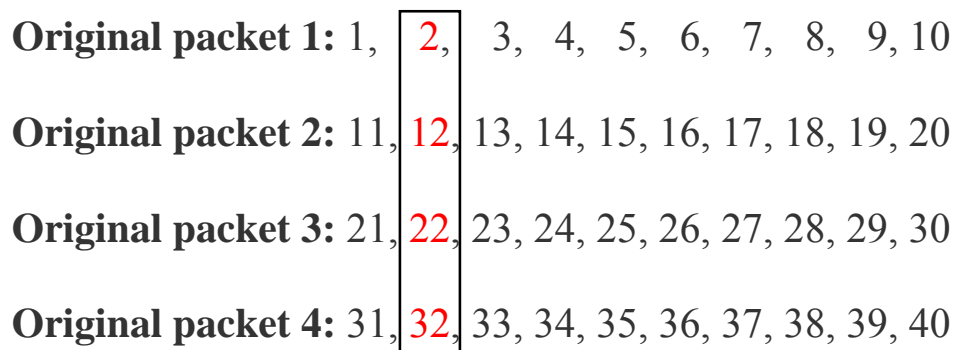

Now the encoded packets consist of a 4-byte coding vector, and 10 bytes encoded data, each with a size of 14 bytes.

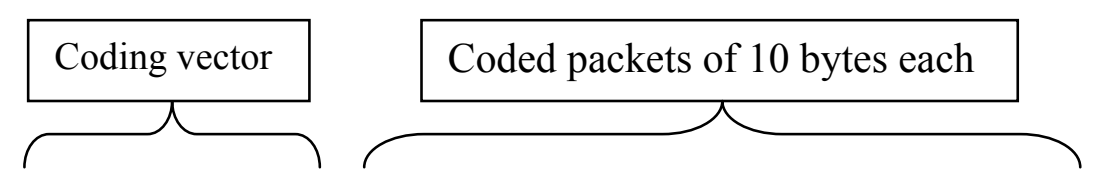

Packet 1: 41, 35, 190, 132, 79, 74, 122, 199, 247, 8, 56, 81, 97, 215

Packet 2: 225, 108, 214, 174, 26, 46, 219, 233, 28, 234, 31, 142, 123, 93

Packet 3: 82, 144, 73, 241, 62, 102, 28, 128, 250, 196, 190, 250, 128, 88

Packet 4: 241, 187, 233, 235, 118, 186, 242, 67, 11, 98, 42, 91, 19, 137

Here, e.g. the data 102 in the box is the 2nd encoded data in Packet 3, which is calculated as below:

(Note: All operations are in $\operatorname{GF}\left(2^{8}\right)$

$\left[\begin{array}{llll}82 & 144 & 73 & 241\end{array}\right] *\left[\begin{array}{c}2 \\ 12 \\ 22 \\ 32\end{array}\right]=82 * 2+144 * 12+73 * 22+241 * 32=\mathbf{1 0 2}$

\section{Re-encoding:}

Note that encoding can be performed recursively to already encoded packets. Consider a node that has already received and stored a set $\left(c_{1}, X_{1}\right), \ldots,\left(c_{M}, X_{M}\right)$ of encoded 
packets. This node may generate a new encoded packet $\left(c^{\prime}, X^{\prime}\right)$ by picking a set of coefficients $h=\left[h_{1}, \ldots, h_{M}\right] \in G F\left(2^{s}\right)$ and computing the linear combination:

$$
X^{\prime}=\sum_{j=1}^{M} h_{j} . X_{j}
$$

It is important that the corresponding encoding vector $c^{\prime}$ is not simply equal to $h$, since the coefficients are with respect to the original packets $B_{1}, B_{2}, \ldots, B_{N}$. Straightforward algebra shows that the new coding vector is given by

$$
c_{i}^{\prime}=\sum_{j=1}^{M} h_{j} \cdot c_{j i}
$$

This operation may be repeated at several nodes in the network. Also note that it is not necessary to decode a packet before applying this type of recursive encoding.

\section{Decoding:}

Assume a node has received the set $\left(c_{1}, X_{1}\right), \ldots,\left(c_{M}, X_{M}\right)$ of encoded packets. In order to retrieve the original packets, it needs to solve the system:

$$
X_{j}=\sum_{i=1}^{N} c_{j i} . B_{i}
$$

The $B_{i}$ are the unknowns. This is a linear system with $M$ equations and $N$ unknowns. We need $M \geq N$ to have a chance of recovering all data, i.e., the number of received packets needs to be at least as large as the number of original packets. This linear system can also be given in a matrix form: $\mathrm{B}=\mathrm{C}^{-1} \times \mathrm{X}$

But the condition $M \geq N$ is not sufficient, as some of the combinations might be linearly dependent. 


\section{Decoding Process [8]:}

Decoding requires solving a set of linear equations. A node stores the received encoded packets as well as its own original packets row by row, into a so-called decoding matrix. Initially this matrix is empty or it contains its own non-encoded packets with their corresponding encoding vectors. When an encoded packet is received, it is inserted as the last row into the decoding matrix. This matrix of coefficients is transformed into a triangular matrix using standard Gaussian elimination (but all operations are performed over the Galois Field). Only innovative packets are inserted. If a packet is non-innovative, it is reduced to a row of 0 s by Gaussian elimination and is ignored. 